\title{
Structure-based Discovery of a Series of NSD2-PWWP1 Inhibitors
}

$\mathrm{Na} \mathrm{Li},{ }^{1,5, \#}$ Hong Yang, ${ }^{2, \#}$ Ke Liu, ${ }^{3, \#}$ Liwei Zhou, ${ }^{1, \#}$ Yuting Huang, ${ }^{2}$ Danyan Cao, ${ }^{1}$ Yanlian Li, ${ }^{1}$ Yaoliang Sun, ${ }^{4}$ Aisong Yu, ${ }^{2}$ Zhiyan Du, ${ }^{1}$ Feng Yu, ${ }^{3}$ Ying Zhang, ${ }^{2}$ Bingyang Wang, ${ }^{4}$ Meiyu Geng, ${ }^{2,6}$ Jian $\mathrm{Li}^{7}$ Bing Xiong, ${ }^{1,5, *}$ Shilin $\mathrm{Xu},{ }^{4, *}$ Xun Huang, ${ }^{2,6, *}$ Tongchao Liu ${ }^{1, *}$

${ }^{1}$ Department of Medicinal Chemistry, Shanghai Institute of Materia Medica, Chinese Academy of Sciences, 555 Zuchongzhi Road, Shanghai 201203, P. R. China

${ }^{2}$ Division of Antitumor Pharmacology, State Key Laboratory of Drug Research, Shanghai Institute of Materia Medica, Chinese Academy of Sciences, 555 Zuchongzhi Road, Shanghai 201203, P. R. China

${ }^{3}$ Shanghai Synchrotron Radiation Facility, Shanghai Advanced Research Institute, Chinese Academy of Sciences, 239 Zhangheng Road, Shanghai 201210, China

${ }^{4}$ Department of Medicinal Chemistry, State Key Laboratory of Drug Research, Shanghai Institute of Materia Medica, Chinese Academy of Sciences, \#555 ZuChong Zhi Road, Shanghai, 201203, China

${ }^{5}$ University of Chinese Academy of Sciences, NO.19A Yuquan Road, Beijing 100049, P. R. China

${ }^{6}$ Hangzhou Institute for Advanced Study, UCAS, Hangzhou 310024, P. R. China;

${ }^{7}$ College of Pharmaceutical Sciences, Gannan Medical University, Ganzhou 341000,

China

${ }^{\#}$ These authors contributed equally 
*Corresponding authors. Tel: +86 2150806600 ext. 5412 fax: +86 2150807088. Email: (T. L.) tongchao_liu@simm.ac.cn; (X.H.) xhuang@simm.ac.cn; (S.X.) slxu@simm.ac.cn; (B. X.) bxiong@simm.ac.cn

\section{ABSTRACT}

Overexpression, point mutations or translocations of protein lysine methyltransferase NSD2 was occurred in many types of cancer cells. Therefore, it was recognized as onco-protein and considered as a promising anticancer drug target. NSD2 consists of a SET catalytic domain and two PWWP domains binding to methylated histone proteins. Here, we reported our efforts to develop a series of NSD2-PWWP1 inhibitors, and further structure-based optimization resulted a potent inhibitor $\mathbf{3 8}$, which has the high selectivity towards NSD2-PWWP1 domain. The detailed biological evaluation revealed that compound $\mathbf{3 8}$ can bind to NSD2-PWWP1 and then affect the expression of genes regulated by NSD2. The current discovery will provide a useful chemical probe to the future research in understanding the specific regulation mode of NSD2 by PWWP1 recognition, and pave the way to develop potential drugs targeting NSD2 protein.

Keywords: Epigenetics; NSD2; PWWP domain; Inhibitor; Structure-based drug discovery

\section{INTRODUCTION}

The nuclear receptor-binding SET domain (NSD) family of protein lysine methyltransferases consists of three members: NSD1, NSD2 and NSD3, and which 
predominantly catalyzes the mono- and di-methylation of histone 3 lysine 36 (H3K36). ${ }^{1}$ High expression, point mutations or translocations of NSD family frequently occurred in multiple types of human cancers. NSD2, also known as MMSET (multiple myeloma SET domain) or WHSC1 (Wolf-Hirschhorn syndrome candidate 1) is involved in several cellular processes, including DNA damage repair, epithelial-mesenchymal transformation (EMT) and cell cycle regulation, and was identified as an important driver in oncogenesis. ${ }^{2}$ Notably, NSD2 is closely related to accelerated disease progression and rapid relapse in $15-20 \%$ of multiple myeloma (MM) harboring the $\mathrm{t}(4 ; 14)$ chromosomal translocation with increased levels of H3K36me2, ${ }^{3}$ and among the most frequently mutated genes in pediatric cancer genomes, E1099K mutant of NSD2 is hyperactivated in acute lymphoblastic leukemia. ${ }^{4}$ Recent studies have revealed that overexpression of NSD2 occurred in several solid tumors including bladder, prostate, glioblastoma and gastrointestinal. ${ }^{5,6}$

NSD2 is a multi-domain protein, consists of the SET domain performing the catalytic methylation function and two PWWP (proline-tryptophan-tryptophan-proline) domains. The N-terminal PWWP domain (PWWP1) acts as the reader domain that preferentially binds to the nucleosomes containing H3K36me2 while PWWP2 domain can bind to histone H3K36/79me3 and dsDNA. Though PWWP1 and PWWP2 are different in affinity to histone substrates, they have been reported to cooperate in maintaining appropriate nuclear localization. This process relies on the conserved aromatic cage consists of PWWP1 Y233, W236 and F266, which are orthogonally positioned and interact with the methylated side chain of H3K36 residue. The 
NSD2-PWWP1:H3K36me2 interaction plays a critical role in stabilizing NSD2 at chromatin, and then facilitates epigenetic spreading and propagation of H3K36me2which is required for the recruitment of DNMT3A and maintenance of DNA methylation at intergenic regions for gene transcription. ${ }^{7}$ In carcinoma cells, H3K36me2 signal alteration underlies epithelial-to-mesenchymal transition and mesenchymal-to-epithelial transition that is critical for metastatic. ${ }^{8}$

Since NSD2 is a promising target for targeted cancer therapy, several groups have reported their efforts in developing selective small-molecule inhibitors targeting the catalytic domain or the PWWP domains of NSD proteins (Figure 1). Huang et al. screened a fragment library of approximately 1600 fragment-like compounds against SET domain of NSD1 with 2D-NMR experiments and found 6-chloro-1,3-benzothiazol-2-amine that binds to the SET domain. ${ }^{9}$ Further structure-based optimization was conducted and resulted to a covalent inhibitor BT5 (1) with moderate selectivity over NSD2 and NSD3. Bottcher et al. from Boehringer Ingelheim company adopted the fragment-based strategy to identify hits to the PWWP1 domain of NSD3. ${ }^{10}$ BI-9321(2) was discovered as a potent and selective inhibitor of NSD3-PWWP1 domain, with $\mathrm{IC}_{50}$ value of $0.2 \mu \mathrm{M}$ in the TR-FRET assay. Coussens et al. utilized the high-throughput screening to identify five small-molecules as inhibitors of the catalytic SET domain and compound $\mathbf{3}$ exhibited apparent activity in cells. ${ }^{11}$ Freitas et al. reported the first inhibitor (4) of NSD2-PWWP1 domain with $\mathrm{K}_{\mathrm{d}}$ value about $3.4 \mu \mathrm{M}$ in an SPR assay, which could abrogate histone H3K36me2 binding to the PWWP1 domain in cells with an $\mathrm{IC}_{50}$ of $17.3 \mu \mathrm{M} .{ }^{12}$ Lately, Dilworth et 
al. continued their previous work and discovered a more potent inhibitor UNC6934 $\left(\mathrm{K}_{\mathrm{d}}=91 \mathrm{nM}\right)$, which harbors same chemical scaffold as compound $\mathbf{4}^{13}$

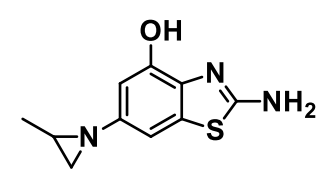

1. BT5 NSD1-SET

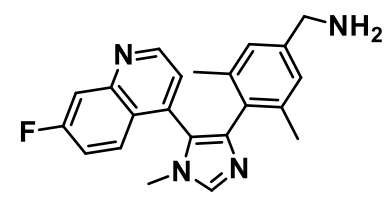

2. $\mathrm{BI}-9321$

NSD3-PWWP1

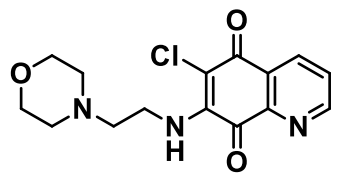

3. DA3003-1 NSD2-SET<smiles>N#Cc1ccc(C(=O)N(Cc2cccs2)C2CC2)cc1</smiles>

4. MR837 NSD2-PWWP1

Figure 1. Representative small-molecule inhibitors of NSD1-3 proteins.

Since there is only one chemotype of NSD2-PWWP1 inhibitors that showed biological activity, therefore, more diverse chemical scaffolds and potent NSD2-PWWP1 inhibitors are needed to further assess the pharmacological potential of NSD2. Herein, we reported our effort to conduct a structure-based optimization of NSD2-PWWP1 ligands, leading to a selective NSD2-PWWP1 inhibitor 38 with significantly improved potency. Compound $\mathbf{3 8}$ can clearly bind to NSD2-PWWP1 and further affect the expression of genes regulated by NSD2. We believe that the discovery and related biological evaluation will contribute to the further research of the specific regulation mode of NSD2 by PWWP1 recognition, and furthermore, our discovery also provided a certain material basis for the functional study of NSD2.

\section{RESULTS AND DISCUSSION}

\section{Rational Design of Selective NSD2-PWWP1 Inhibitors.}

At the initial stage of this project, we analyzed the binding model of reported small-molecule compounds to understand the binding basis between inhibitors and proteins, which enabled us to rationally design a new class of NSD2 inhibitors. 
Currently, the crystal structure of NSD2-PWWP1 domain bound with MR837 was reported (PDB ID: 6UE6) ${ }^{12}$, which revealed two important binding features: 1) N-cyclopropyl amide of MR837 occupied the aromatic cage sub-pocket forming by three conservative aromatic residues, Tyr233, Trp236 and Phe266; 2) an essential hydrogen bonding interaction between Ala270 residue and the $\mathrm{N}$ atom of benzonitrile. As mentioned above, BI-9321, the first selective and potent PWWP inhibitor in NSD family was reported by Bottcher et al. and demonstrated in the cocrystal structure (PDB code: $6 \mathrm{G} 2 \mathrm{O})^{10}$, binds to NSD3-PWWP1 domain by the following patterns: the aromatic cage was occupied by $\mathrm{N}$-methylimidazole ring; the $\mathrm{N}$ atom in the quinoline ring interacted with Ser314 through hydrogen bonding; and the $\mathrm{NH}_{2}$ of benzylamine extending out the binding pocket and forming a strong electrostatic interaction with nearby negative charged residue Glu318.

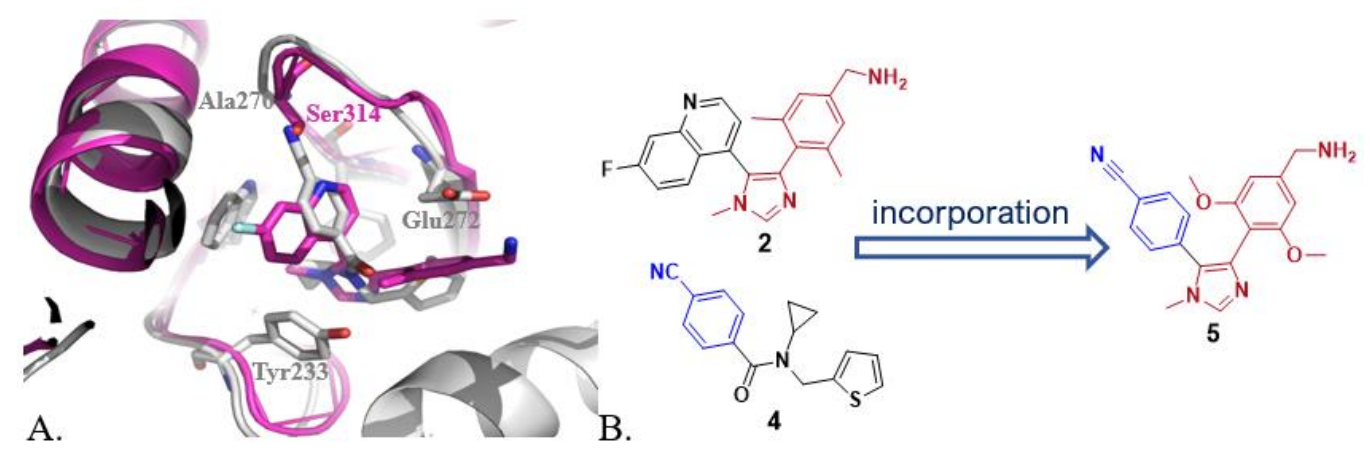

Figure 2. Design of NSD2-PWWP1 inhibitor. (A) The superimposition of crystal structures of PWWP inhibitors of NSD2-PWWP1 (MR837, PDB code 6UE6, colored gray) and NSD3-PWWP1 (BI-9321, PDB code 6G2O, colored purple). (B) Design of new chemotype of NSD2-PWWP1 inhibitor 5.

By superposition of these two cocrystal structures (Figure 2A), it could be seen that, comparing the binding site of NSD3-PWWP1 domain with the NSD2-PWWP1 
domain, the most striking difference is at the vicinity of benzonitrile group of MR837. In the NSD2-PWWP1 domain, there are Gly268, Asp269 and Ala270 residues forming hydrogen bonds with benzonitrile structure, while in NSD3-PWWP1 domain, at the same position, there are Ser314, Asn315 and Gln316 surrounding the quinolone ring of BI-9321. It should be noted that the side chain of Ser314 forming a hydrogen bond with the nitrogen atom of quinoline, which would impede the benzonitrile group binding. Therefore, we rationally designed the new chemotype of NSD2-PWWP1 inhibitors by incorporating the benzonitrile into the BI-9321 to replace the quinoline ring, which was thought that could achieve the binding selectivity of NSD2 over the NSD3 protein. Besides, we also noticed that the methyl group on the benzene ring of BI-9321 was at the similar position of the $\mathrm{O}$ atom of the amide group of MR837, and the distance between the methyl $\mathrm{C}$ atom on the benzene ring of BI-9321 and Tyr233 was 3.6 A. We hypothesized that if the methyl on the benzene ring was replaced with methoxy group, the $\mathrm{O}$ atom of methoxyl group may be able to form a hydrogen bond with Tyr233. Taking together, we designed and synthesized compound $\mathbf{5}$ in Figure 2B for the biological test to verify the design strategy.

\section{Structure-Activity Relationship (SAR) Exploration and Optimization}

\section{of Ligands.}

As shown in Table 1, compound $\mathbf{5}$ with two methoxy groups displayed a good binding affinity to NSD2 PWWP1 domain, with an $\mathrm{IC}_{50}$ value of $4.44 \mu \mathrm{M}$, which is much better than MR837 (4). This encouraged us to synthesize analogs 6 - 12. According to the binding assay, removal of one or two methoxy groups led to compounds $\mathbf{6}$ and $\mathbf{7}$ 
showing slightly reduced potency with $\mathrm{IC}_{50}$ values of $11.97 \mu \mathrm{M}$ and $13.95 \mu \mathrm{M}$, respectively. When mono-halogen groups such as chlorine and fluorine were introduced (compound $\mathbf{8}$ and $\mathbf{9}$ ), they also slightly reduced potency if comparison with compound 5. However, single trifluoromethyl-substituent and methyl-substituent compounds (10 and 11) showed equal potency to compound 5. When another methyl group was introduced to yield compound $\mathbf{1 2}$, the potency was significantly improved to an $\mathrm{IC}_{50}$ value of $0.57 \mu \mathrm{M}$, about 8 -fold more potent than of compound $\mathbf{5}$. This encouraged us to further explore the effect of other parts of this chemotype inhibitors on NSD2-PWWP1 binding.

Table 1. NSD2 Inhibition of Compounds 5 - 12 Combining $R^{1}$ - and $R^{2}$ Substituents $^{a}$

\begin{tabular}{|c|c|c|c|}
\hline compound & $\mathbf{R}^{1}=$ & $\mathbf{R}^{2}=$ & NSD2 $\left(\mathrm{IC}_{50}, \mu \mathrm{M}\right)$ \\
\hline 5 & $\mathrm{OCH}_{3}$ & $\mathrm{OCH}_{3}$ & $4.44 \pm 3.30$ \\
\hline 6 & $\mathrm{OCH}_{3}$ & $\mathrm{H}$ & $11.97 \pm 3.64$ \\
\hline 7 & $\mathrm{H}$ & $\mathrm{H}$ & $13.95 \pm 6.73$ \\
\hline 8 & $\mathrm{Cl}$ & $\mathrm{H}$ & $6.11 \pm 3.38$ \\
\hline 9 & $\mathrm{~F}$ & $\mathrm{H}$ & $11.19 \pm 2.80$ \\
\hline 10 & $\mathrm{CF}_{3}$ & $\mathrm{H}$ & $6.01 \pm 2.02$ \\
\hline 11 & $\mathrm{CH}_{3}$ & $\mathrm{H}$ & $4.68 \pm 2.12$ \\
\hline
\end{tabular}




\begin{tabular}{cccc}
12 & $\mathrm{CH}_{3}$ & $\mathrm{CH}_{3}$ & $0.57 \pm 0.12$ \\
& & & \\
$\mathrm{MR} 837$ & - & - & $24.67 \pm 1.86$ \\
\hline
\end{tabular}

${ }^{a} \mathrm{IC}_{50}$ values are shown as the mean $\pm \mathrm{SD}$ from at least two separate determinations.

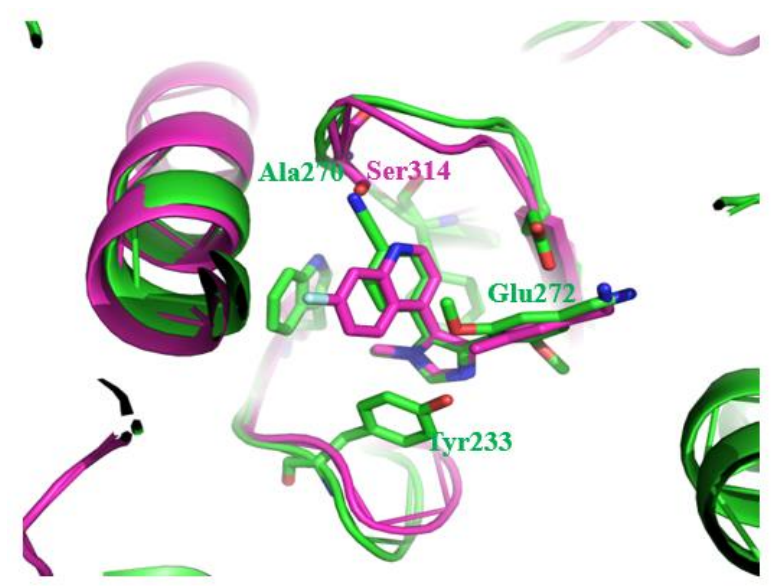

Figure 3. Superimposition of the crystal structure of NSD2-PWWP1 domain with compound 5 (colored green, PDB ID:7VLN) and the crystal structure of NSD3-PWWP1 bound with BI-9321 (colored purple, PDB ID: 6G2O).

To verify the design, we performed the crystallization experiment and solved the cocrystal structure of NSD2-PWWP1 domain with compound 5. As illustrated in Figure 3, compound 5 bound to the NSD2 in a mode very similar to BI-9321 bound to the NSD3: the N-methylimidazole situated into the aromatic cage and the benzylamine group interacted with the nearby Glu272; while the benzonitrile motif represents as an essential pharmacophore for PWWP1 of NSD2, which, as predicted, interacts with residues Asp269 and Ala270. Clearly, the nitrile group would be collided with the serine residue in NSD3.

Based on this solved structure, we next conducted a series of structural modifications to get compounds $\mathbf{1 3}-\mathbf{2 0}$ to further explore the interactions with this 
benzonitrile binding subpocket. As shown in Table 2, replacement of the benzonitrile core of compound $\mathbf{1 2}$ with a naphthonitrile moiety led to compound $\mathbf{1 3}$ showing analogous potency with an $\mathrm{IC}_{50}$ value of $0.52 \mu \mathrm{M}$. Further replacement of the naphthalene moiety with a quinoline moiety generated compound $\mathbf{1 4}$ showing an $\mathrm{IC}_{50}$ value of $1.58 \mu \mathrm{M}, 3$-fold less potent than compound 13. Introducing an amino- or methylamino-substituent in the ortho-position of cyano group of compound $\mathbf{1 2}$ reduced the potency (compound 15 and 16). Introduction of other small substituents afforded compounds 17 - 20. Unfortunately, all these compounds exhibited less activity against NSD2. These results indicate that limited structural variations are tolerated in this region and the naphthonitrile framework remains to be advantageous to interact with NSD2. Because compound $\mathbf{1 3}$ is slightly more potent, and protein thermal shift assay (TSA) experiment further confirmed that compound $\mathbf{1 3}$ can selectively bind to NSD2-PWWP1 protein more tightly than compound $\mathbf{1 2}$ (Figure S1), we selected compound $\mathbf{1 3}$ for further structural optimization with the central naphthonitrile core intact.

Table 2. NSD2 Inhibition of Compounds Bearing Different Aromatic

\section{Cyano-Fragments $^{a}$}

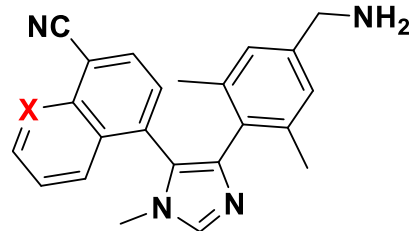

13: $X=C ; 14: X=N$

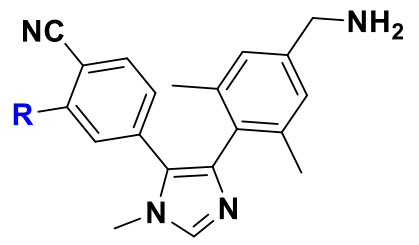

$15-20$

compound $\quad \mathbf{R}=\quad \mathbf{N S D 2}\left(\mathrm{IC}_{50}, \mu \mathrm{M}\right)$


14

15

16

17

18

19

20
$\mathrm{NH}_{2}$

$\mathrm{NHCH}_{3}$

F

$\mathrm{Cl}$

$\mathrm{OCH}_{3}$

$\mathrm{CF}_{3}$
$1.58 \pm 0.85$

$8.78 \pm 2.18$

$>20$

$2.91 \pm 0.13$

$5.03 \pm 2.60$

$10.93 \pm 7.33$

$15.41 \pm 1.82$

${ }^{a} \mathrm{IC}_{50}$ values are shown as the mean \pm SD from at least two separate determinations.

From the cocrystal structure of NSD3-PWWP1 in complex with BI-9321, we can learn that the benzylamine group interacts with Glu318 residue by a hydrogen bond and this residue is conserved in the PWWP1 domain of NSD2. We synthesized and tested compounds bearing different hydrogen bond groups as the replacement of the benzylamine group. As shown in Table 3, almost all these compounds lost activity against NSD2 by showing $\mathrm{IC}_{50}$ values greater than $20 \mu \mathrm{M}$. Notably, compounds $\mathbf{2 4}$ and 27 showed much reduced potency with $\mathrm{IC}_{50}$ values of $13.81 \mu \mathrm{M}$ and $10.56 \mu \mathrm{M}$, respectively. These results indicated that benzylamine group was necessary for activity against NSD2 and minor modifications led to much reduced potency.

Table 3. NSD2 Inhibition of Compounds Bearing Different H-bond Donor

\section{Groups $^{a}$}

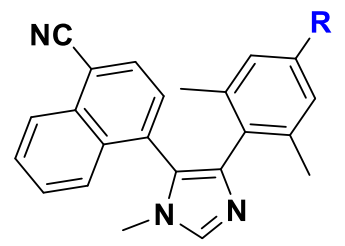




\begin{tabular}{|c|c|c|}
\hline compound & $\mathbf{R}=$ & NSD2 $\left(\mathrm{IC}_{50}, \mu \mathrm{M}\right)$ \\
\hline 21 & $\mathrm{CN}$ & $>20$ \\
\hline 22 & $\mathrm{CHO}$ & $>20$ \\
\hline 23 & $\mathrm{COCH}_{2} \mathrm{CH}_{3}$ & $>20$ \\
\hline 24 & $\mathrm{CH}_{2} \mathrm{OH}$ & $13.81 \pm 0.47$ \\
\hline 25 & $\mathrm{CHOHCH}_{2} \mathrm{CH}_{3}$ & $>20$ \\
\hline 26 & NHBoc & $>20$ \\
\hline 27 & $\mathrm{NH}_{2}$ & $10.56 \pm 3.31$ \\
\hline
\end{tabular}

${ }^{a} \mathrm{IC}_{50}$ values are shown as the mean \pm SD from at least two separate determinations.

During the exploration of SARs, we found that tert-butoxycarbonyl-protected compound 13 , compound 28 still showed moderate potency with an $\mathrm{IC}_{50}$ value of 1.67 $\mu \mathrm{M}$. This indicated that there is certain space around this binding subsite to conduct structural modifications. Therefore, we further explored steric and hydrophilic tolerance by introducing bulky alkyl- or water-soluable substituents. As shown in Table 4, The $N$-ethyl substituted compound 29, $N$-cyclopropyl substituted compound $\mathbf{3 0}$ and $N$-tert-butyl substituted compound $\mathbf{3 1}$ displayed increased potency with $\mathrm{IC}_{50}$ values of $0.70 \mu \mathrm{M}, 0.43 \mu \mathrm{M}$ and $0.64 \mu \mathrm{M}$, respectively, which were equivalent to compound 13. When the nitrogen was double substituted to afford compounds $\mathbf{3 2}$ - $\mathbf{3 6}$, they showed decreased potency with $\mathrm{IC}_{50}$ values ranging from $1.49 \mu \mathrm{M}$ to $8.07 \mu \mathrm{M}$. However, removal of Boc protection led to significantly increased potency (compound 37 and 38), with an $\mathrm{IC}_{50}$ value of $1.89 \mu \mathrm{M}$ and $0.11 \mu \mathrm{M}$, respectively. Introduction of a hydroxyl group at the C3-position of compound 32 led to 4-fold 
increased potency (compound 39), while the dimethylamino group led to retained potency (compound 40) compared to corresponding compound 32. Replacement of the amino piperidine of compound $\mathbf{3 8}$ with an ethylenediamine yielded compound $\mathbf{4 1}$ showing 6-fold reduced potency. Notably, among these compounds, compound $\mathbf{3 8}$ showed the best potency against NSD2, 5-fold more potent than compound $\mathbf{1 3}$.

Table 4. NSD2 Inhibition of Compounds Bearing Variable Amino Substituents ${ }^{a}$

\begin{tabular}{|c|c|c|}
\hline compound & $\mathbf{R}=$ & NSD2 $\left(\mathrm{IC}_{50}, \mu \mathrm{M}\right)$ \\
\hline 13 & & $0.52 \pm 0.20$ \\
\hline 28 & & $1.67 \pm 0.41$ \\
\hline 29 & & $0.70 \pm 0.12$ \\
\hline 30 & & $0.43 \pm 0.01$ \\
\hline 31 & & $0.64 \pm 0.10$ \\
\hline 32 & & $2.07 \pm 0.43$ \\
\hline 33 & & $1.49 \pm 0.16$ \\
\hline 34 & & $3.84 \pm 1.60$ \\
\hline 35 & & $8.07 \pm 2.37$ \\
\hline
\end{tabular}




36

${ }^{a} \mathrm{IC}_{50}$ values are shown as the mean $\pm \mathrm{SD}$ from at least two separate determinations.

\section{Biological Evaluation}

\section{Selectivity profile with Protein Thermal shift}

We carried out protein thermal shift assay to directly confirm the binding between NSD2-PWWP protein and compound 38. As shown in Figure 4, compared to DMSO control, the melting temperature (Tm values) of NSD2-PWWP1 protein was evidently increased due to the addition of compound $\mathbf{3 8}$ and the change of melting temperature ( $\mathrm{Tm}$ ) in a dose-dependent manner at concentrations from $0.781 \mu \mathrm{M}$ to $200 \mathrm{mM}$. The results indicated that the ligands directly bind to NSD2-PWWP protein and could strengthen its stability in vitro. We further evaluated the target binding specificity of compound $\mathbf{3 8}$ using the thermal shift assay. Our results show that compound $\mathbf{3 8}$ exhibited remarkable selectivity against NSD2-PWWP1 protein over other PWWP domains protein (NSD3-PWWP1, DNMT3A-PWWP, ZCWPW1-PWWP). 

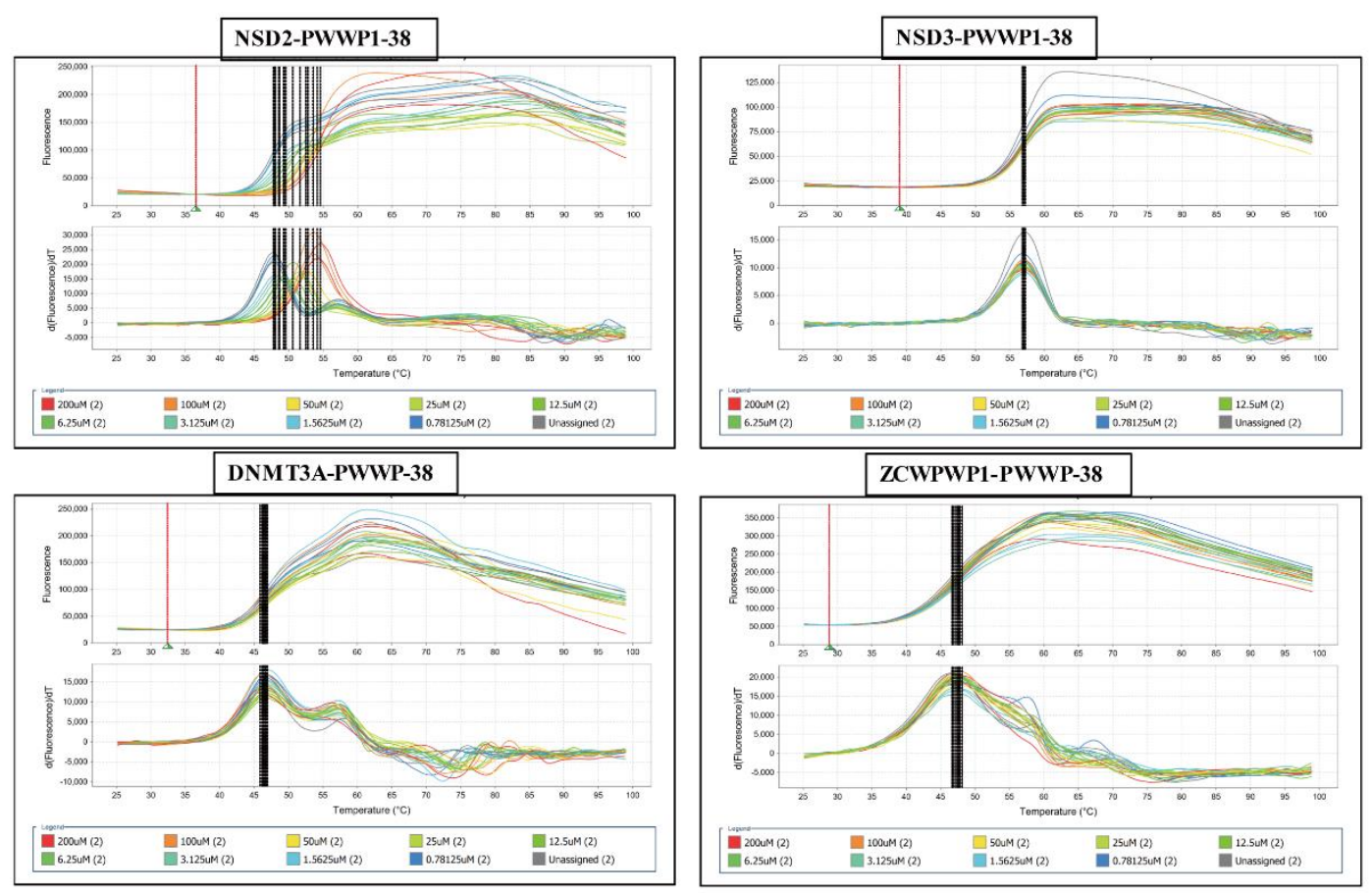

Figure 4. The melting curves of PWWP domains protein protein with compound $\mathbf{3 8}$. The thermal shift assay displayed that treatment with compound $\mathbf{3 8}$ increases the melting temperature. Compound $\mathbf{3 8}$ exhibited remarkable selectivity against NSD2-PWWP1 protein over other PWWP domains protein (NSD3-PWWP1, DNMT3A-PWWP, ZCWPW1-PWWP).

\section{Target validation at the molecular and cellular level}

We proved in the previous section that compound $\mathbf{3 8}$ could bind to NSD2-PWWP1 domain. To further detect whether compound $\mathbf{3 8}$ can affect HK36me2, we adopted AlphaLisa which was designed for screening NSD2 enzymatic inhibitors. It was shown that compound $\mathbf{3 8}$ did not display inhibition effect on $\mathrm{H} 3 \mathrm{~K} 36 \mathrm{me} 2$ in vitro (Figure 5A). Consistent with Alphalisa results, compound 38 caused little effect on general H3K36me2 in cellular level (Figure 5B). These results are consistent with the findings from the study of Dilworth et al., as they pointed out that PWWP1 antagonism by UNC6934 did not have a significant effect on ribosome transcription 
or global levels of H3K36me2. ${ }^{13}$ In conclusion, compound $\mathbf{3 8}$ had no impact on SET domain or enzyme activity catalytic function, which further validated that compound 38 could specifically binds to PWWP domain.

A

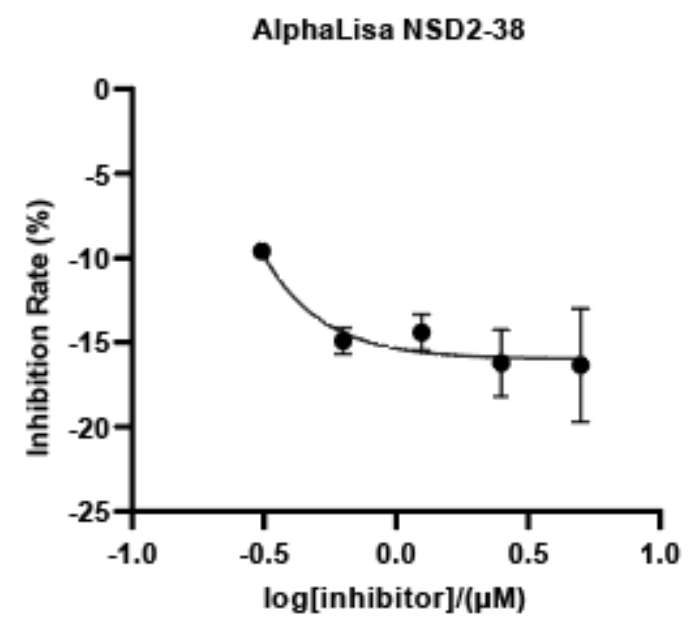

B

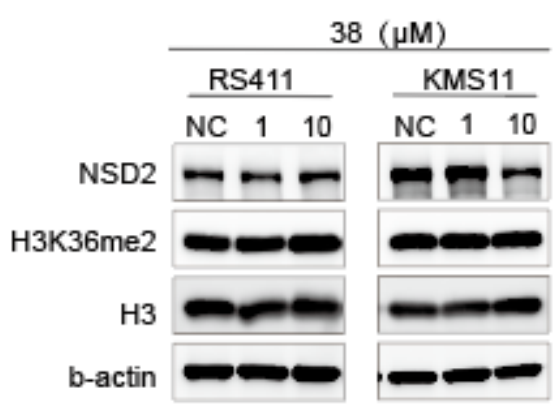

Figure 5. Target validation of compound $\mathbf{3 8}$ in molecular and cellular level. (A) The inhibition of compound $\mathbf{3 8}$ on the H3K36me2 level via Alphalisa screening. (B) RS4:11and KMS11 cells were treated with compound $\mathbf{3 8}$ at gradient concentrations for 3 days, and the protein level of H3K36me2 was detected by western blot. $\beta$-actin was used as a loading control. Data are reported as the mean \pm SD.

\section{NSD2-positive correlated downstream genes validation}

To examine the cellular pathways affected by compound 38, we performed RNA sequencing (RNA-seq) on the sensitive cell line KMS-11 by treating the cells with compound 38 or DMSO as a control for $72 \mathrm{~h}$. By the KEGG analysis of the RNA-Seq, multiple cellular pathways are changed upon compound 38. Numerous genes were adjusted up or down (Figure 6A and Figure 6B). 


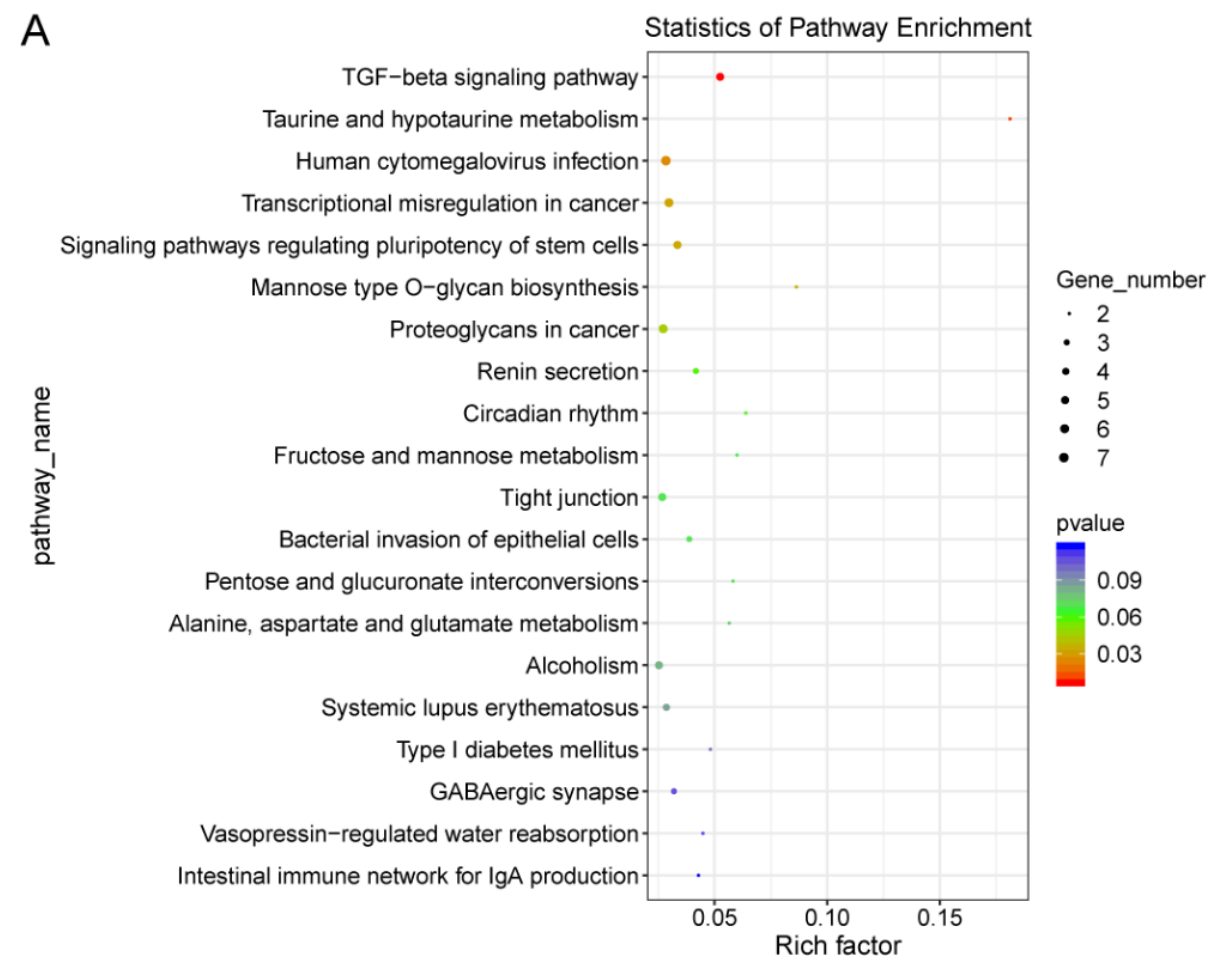

B

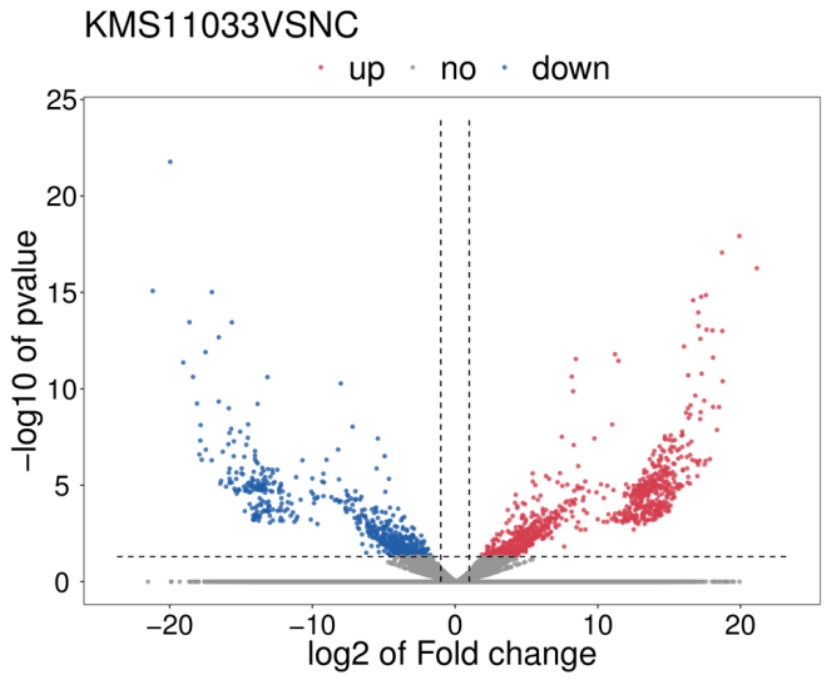

Figure 6. (A) KEGG analysis of the differential genes upon compound $\mathbf{3 8}$ treatment identified by RNA-seq in KMS-11 cells. (B) Volcano plot showing the differentially expressed genes in KMS-11 cells treated with compound $38(8 \mu \mathrm{M})$ for $72 \mathrm{~h}$.

We next determined whether compound $\mathbf{3 8}$ could affect NSD2 correlated downstream genes. The expression of several published representative genes(PAK1, RRAS2, TCFA, TEMEL2, HSPG2, NCAM1) were validated in two cancer cell lines by 
qRT-PCR analysis. Six pairs of qPCR primers were designed. RT-PCR results showed that NSD2 target genes PAK1, RRAS2, TGFA, TEMEL2, NCAM1 remarkably decreased in compound $\mathbf{3 8}$ treatment group compared with control in MV4;11 and KMS11 cell lines, except for HSPG2. These results confirmed compound $\mathbf{3 8}$ could affect NSD2 correlated downstream genes changes (Figure 7). A previous study indicate that NSD2-PWWP1 mutation can slightly reduce H3K36me2 level. ${ }^{14}$ However, we found that interruption of NSD2 and H3K36me2 can downregulate gene transcription without influence of H3K36me2 level. These results confirm a multiple function protein of NSD2 according to its structure.
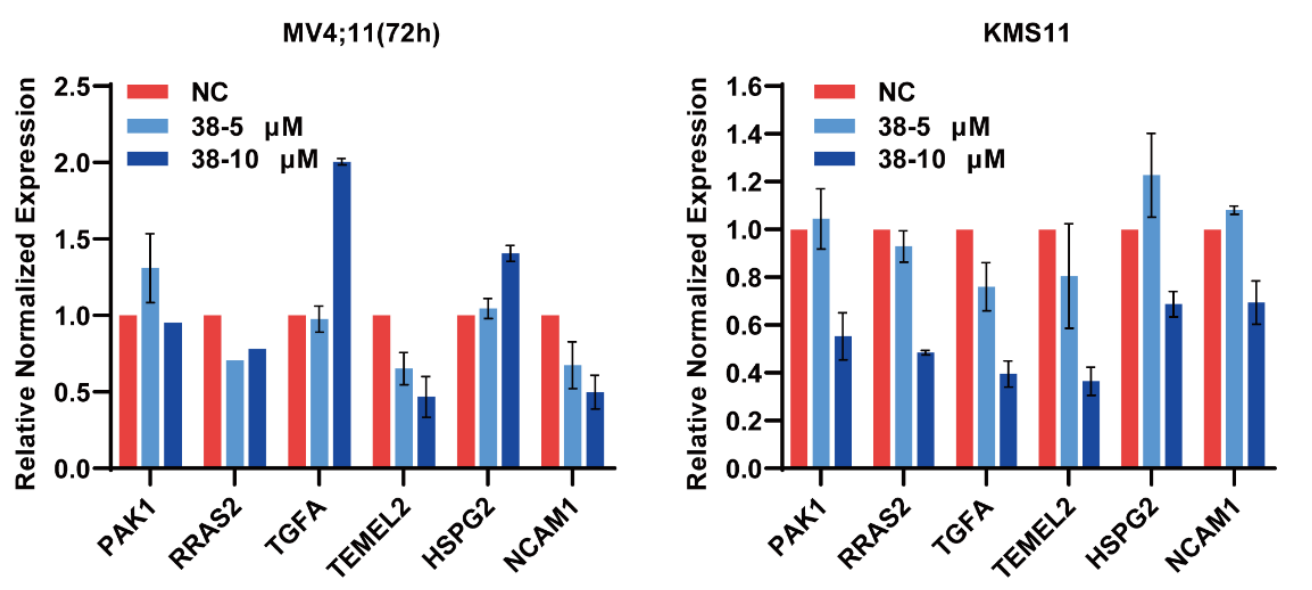

Figure 7. Downstream oncogenic transcriptional programs of compound 38. The effects of compound $\mathbf{3 8}$ on gene expressions in 2 cell lines. The cell lines were treated with vary concentrations of compound $\mathbf{3 8}$ for 3 days. Data are reported as the mean \pm SD.

\section{Cell-based activity}

We then assessed the potential antitumor activity of compound $\mathbf{3 8}$ in vitro, we evaluated its effects on cell proliferation in a panel of human cell lines. The results demonstrated that compound $\mathbf{3 8}$ inhibited cell proliferation in these cell lines, including the cell line RS4:11 $\left(\mathrm{IC}_{50}=6.30 \mu \mathrm{M}\right), \mathrm{MV} 4 ; 11\left(\mathrm{IC}_{50}=2.23 \mu \mathrm{M}\right), \mathrm{KMS} 11$ 
$\left(\mathrm{IC}_{50}=8.43 \mu \mathrm{M}\right)$, and MM1S $\left(\mathrm{IC}_{50}=10.95 \mu \mathrm{M}\right)$. (Figure 8). Our previous study reported a NSD2-SET domain inhibitor 9c which showed great inhibitory effect in NSD2 mutant cells KMS11 and RS4:11 ( $\mathrm{IC}_{50}$ were $0.52 \mu \mathrm{M}$ and $1.88 \mu \mathrm{M}$, respectively). However, NSD2 WT cell MV4;11 showed little response to $9 \mathrm{c}^{15}$ In this study, NSD2-PWWP1 inhibitor 38 showed inhibitory effect in both NSD2 mutant and wild type cells. Therefore, we speculated that NSD2-PWWP1 inhibitor inhibited cell proliferation independent of NSD2 genotype, which was different from NSD2-SET inhibitor.

38

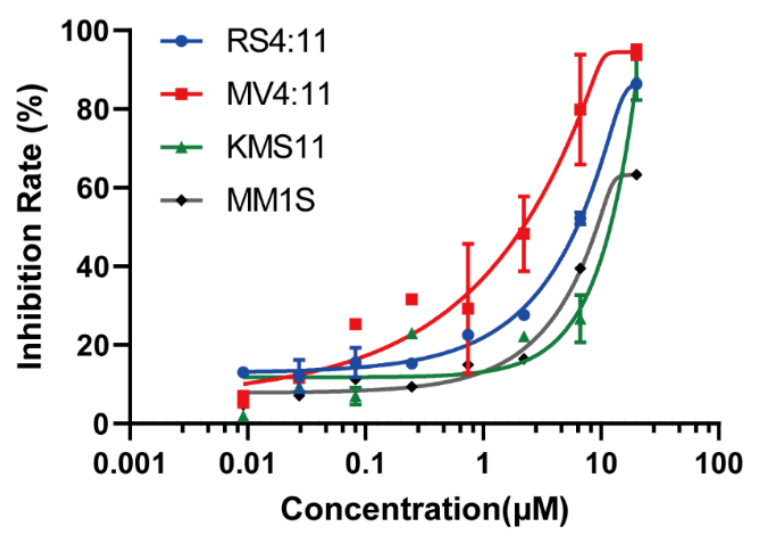

Figure 8. Cellular activity of compound 38. The inhibitory effects on the growth of 4 cell lines treated with compound $\mathbf{3 8}$ at gradient concentrations for 6 days, and the inhibitory rate was measured using the CCK8 Kit. Data are reported as the mean \pm SD.

\section{compound 38 induces apoptosis and cell cycle arrest}

Next, we further investigated the effects of compound $\mathbf{3 8}$ on cell cycle progression and apoptosis. The effects of compound $\mathbf{3 8}$ on cell cycle progression and apoptosis were evaluated in KMS11 and MV4;11 cells. We treated the KMS11 and MV4;11 cells with vehicle alone or compound 38 at 5 and $10 \mu \mathrm{M}$ for $72 \mathrm{~h}$ and 
evaluated by flow cytometry. A higher proportion of apoptotic cells was observed after compound 38 treatment among the KMS11 and MV4:11 cells and the apoptotic effect is dose-dependent (Figure 9A). Moreover, cell cycle analysis showed that treatment of compound $\mathbf{3 8}$ in the KMS11, and MV4;11 cells increased the proportions of cells in G0/G1phase whereas decreased the proportions of cells in $\mathrm{S}$ and G2/M phase (Figure 9B).
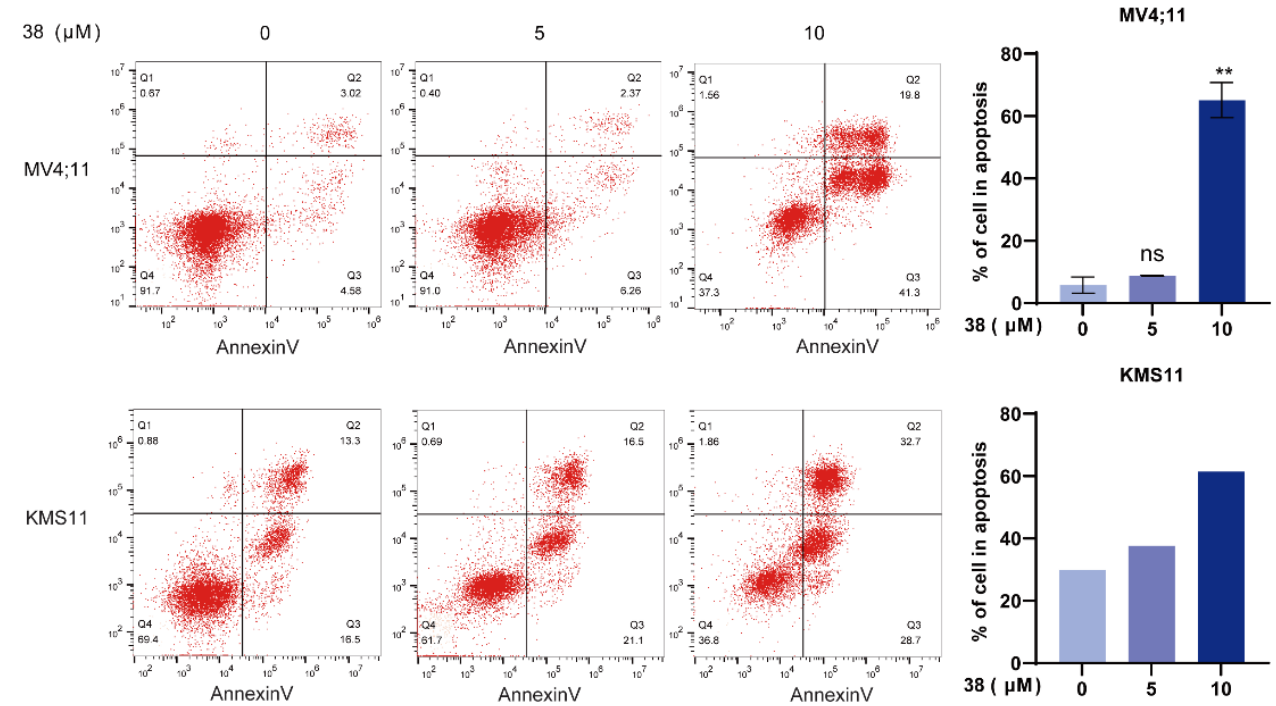

B
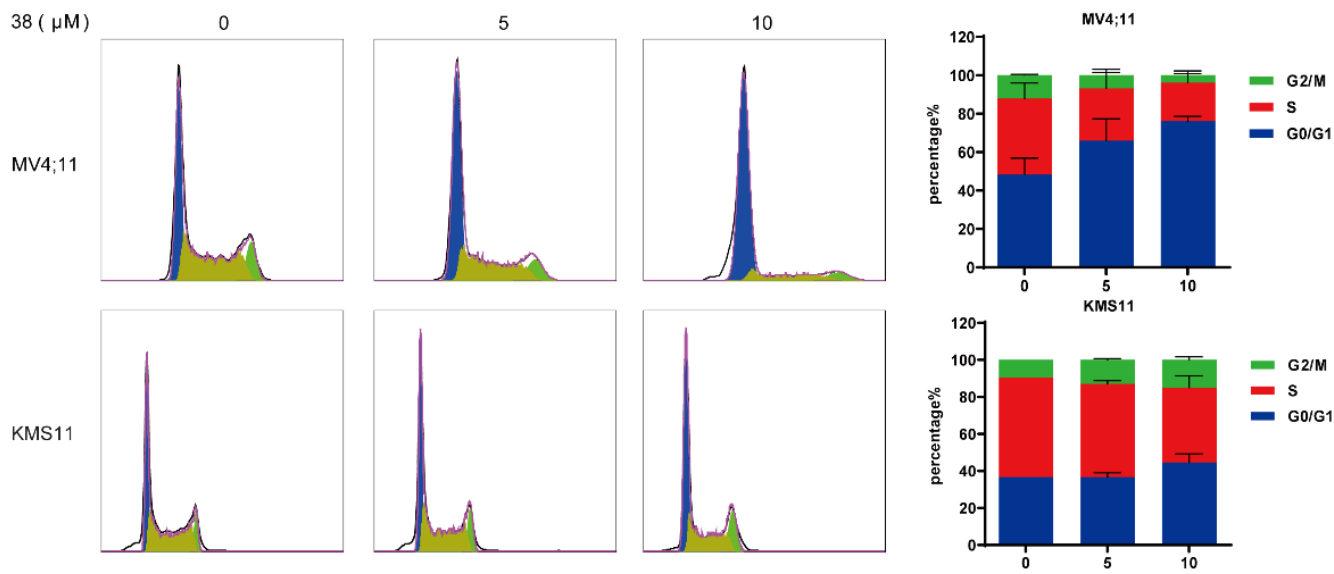

Figure 9. compound 38 induces cell apoptosis and arrests the cell cycle at the G0/G1 phase in MV4;11 and KMS11 cells. (A) KMS11 and MV4:11 cells were treated with various concentrations 38 (at 5 and $10 \mu \mathrm{M}$ ) for $72 \mathrm{~h}$, and the effects on apoptosis were examined by flow cytometry. (B) KMS11 and MV4:11 cells were incubated with 38 (at 5 and $10 \mu \mathrm{M}$ ) for $24 \mathrm{~h}$, and the the percentage of each cell cycle 
phase were determined by flow cytometry.

\section{CHEMISTRY}

The synthesis of compounds $\mathbf{5}-\mathbf{1 2}$ is depicted in Scheme 1. The starting materials 1-methyl-1H-imidazole (42) and 4-bromobenzonitrile (43) were coupled to afford intermediate 44 via palladium acetate-catalyzation. The intermediate 44 was reacted with NBS to provide the key intermediate 45 , which was then subjected to Suzuki coupling with corresponding boronic acid or boronic acid pinacol ester to provide intermediates $46-48$ and $49-53$. Finally, the intermediates $46-48$ underwent reductive amination reaction to obtain the target compounds 5 - 6 and $\mathbf{1 2}$, and the target compounds $\mathbf{7}$ - $\mathbf{1 1}$ were achieved from $\mathbf{4 9}$ - $\mathbf{5 3}$ by Boc-deprotection.

The synthesis of compounds $\mathbf{1 3}-\mathbf{1 4}$ and $\mathbf{2 8}$ were similar to those described for $\mathbf{5}$ and 7, as illustrated in Scheme 2. The reaction of 1-methyl-1H-imidazole (42) and 59a-b produced intermediates $\mathbf{6 0 a - b}$, which was further treated with NBS to afford intermediates 61a-b. The target compound 28 was obtained by Suzuki coupling of 61a and 58e, and the target compound 13 was achieved by Boc-deprotection of compound 28. Coupling of $\mathbf{6 1 a}$ with $\mathbf{5 5 c}$, followed by reductive amination produced the target compound 14 .

The synthesis of compounds $\mathbf{1 5}$ and $\mathbf{1 6}$ is shown in Scheme 3. The commercially available 2-amino-4-bromobenzonitrile (64a) was converted to intermediate $\mathbf{6 4 b}$ by treatment with iodomethane. The starting materials 4-bromo-1-methyl- $1 \mathrm{H}$-imidazole (63) was coupled with 4-bromobenzonitrile derivatives (64a-b) to afford intermediates $\mathbf{6 5 a} \mathbf{a}-\mathbf{b}$ via palladium acetate-catalyzation. The intermediates $\mathbf{6 5 a} \mathbf{a}-\mathbf{b}$ were 
subjected to Suzuki coupling with boronic acid pinacol ester (58e), and the resulting 66a-b were deprotected by $\mathrm{HCl}$ in dioxane to afford compounds $\mathbf{1 5}$ and $\mathbf{1 6 .}$

The general synthesis of compounds $\mathbf{1 7}-\mathbf{2 0}$ was outlined in Scheme 4. The commercially available 5-bromo-1-methyl-1H-imidazole (67) was coupled with various boronic acids or boronic acid pinacol esters, and the resulting 68a-d were treated with NBS to yield the intermediates 69a-d. Another Suzuki coupling reaction was conducted between the intermediates 69a-d and 58e, followed by Boc-deprotection in TFA to obtain the target compounds $\mathbf{1 7}-\mathbf{2 0}$.

The general synthesis of compounds 21 - 27 was described in Scheme 5. The intermediate 61a prepared in Scheme 2 was subjected to Suzuki coupling with appropriate boronic acid pinacol esters to produce the target compounds $\mathbf{2 1}-\mathbf{2 3}$ and 26. The compounds 22 and $\mathbf{2 3}$ were further reduced by $\mathrm{NaBH}_{4}$ to yield compounds $\mathbf{2 4}$ and 25, respectively. The target compound 27 was conveniently obtained from deprotection of compound $\mathbf{2 6}$.

The general synthesis of compounds 29 - 41 was outlined in Scheme 6. The compound 22 bearing aldehyde group was subjected to reductive amination with various amines to afford the desired compounds $29-\mathbf{3 6}, 39-40$ and $\mathbf{7 2}$. Subsequently, the target compounds $\mathbf{3 7}-\mathbf{3 8}$ and $\mathbf{4 1}$ were achieved by deprotection of Boc groups of compounds $\mathbf{3 5}-\mathbf{3 6}$ and $\mathbf{7 2}$.

Scheme 1. Synthesis of Compounds 5-12 


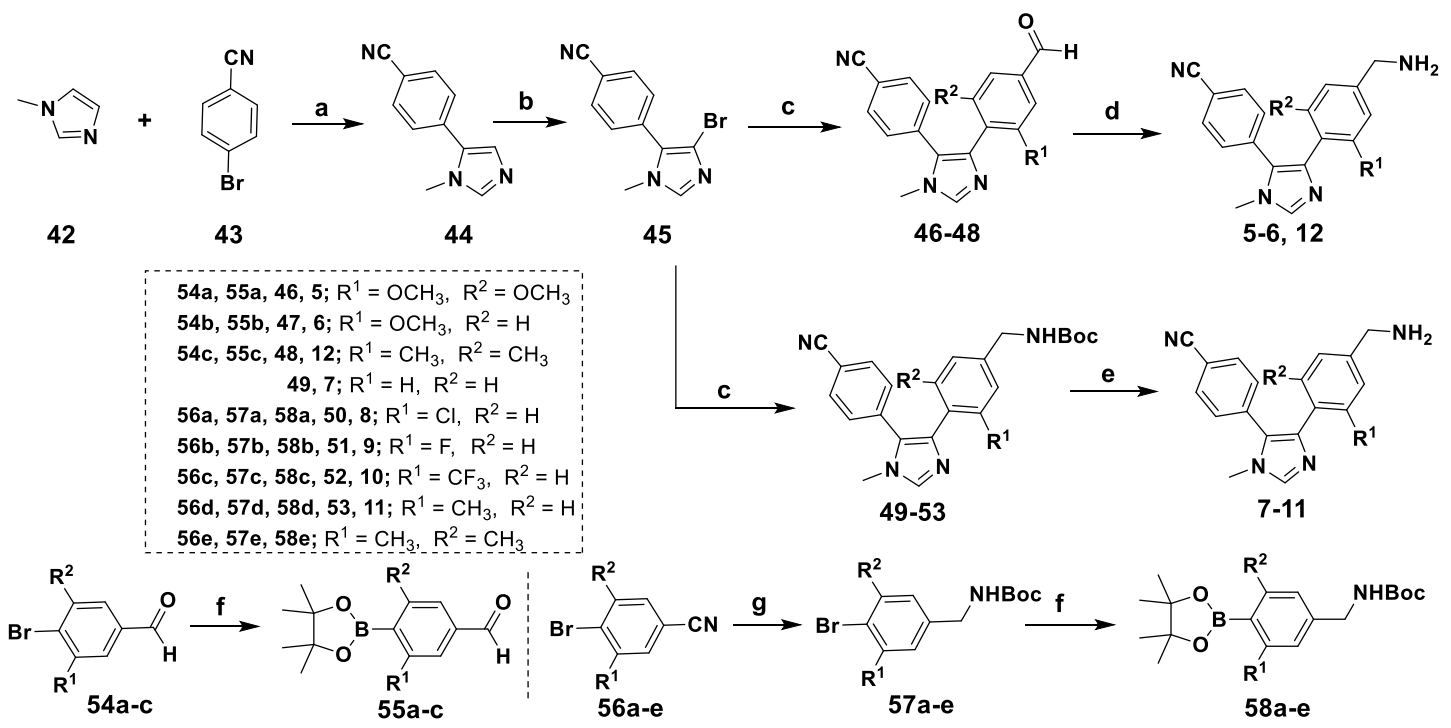

${ }^{a}$ Reagents and conditions: (a) $\mathrm{Pd}(\mathrm{OAc})_{2}$, KOAc, DMAc, $120{ }^{\circ} \mathrm{C}, 10 \mathrm{~h}$; (b) NBS, $\mathrm{CH}_{3} \mathrm{CN}, 0{ }^{\circ} \mathrm{C}, 10$ h; (c) 55a-c, $\mathrm{PdCl}_{2}(\mathrm{dppf}), \mathrm{K}_{2} \mathrm{CO}_{3}$, dioxane/ $\mathrm{H}_{2} \mathrm{O}, 100{ }^{\circ} \mathrm{C}, 7 \mathrm{~h}$; 58a-d, XPhos Pd G2, $\mathrm{K}_{3} \mathrm{PO}_{4}$, dioxane/ $\mathrm{H}_{2} \mathrm{O}, 90{ }^{\circ} \mathrm{C}$, overnight; (d) $\mathrm{CH}_{3} \mathrm{COONH}_{4}, \mathrm{CH}_{3} \mathrm{COOH}, \mathrm{NaBH}_{3} \mathrm{CN}, \mathrm{MeOH}, 0{ }^{\circ} \mathrm{C}, 8 \mathrm{~h}$; (e) trifluoroacetic acid, $\mathrm{CH}_{2} \mathrm{Cl}_{2}, \mathrm{RT}, 1 \mathrm{~h}$; (f) Bis(pinacolato)diboron, $\mathrm{PdCl}_{2}$ (dppf), $\mathrm{AcOK}$, dioxane, 80 ${ }^{\circ} \mathrm{C}, 8 \mathrm{~h}$; and (g) for 57a-d: $\mathrm{BH}_{3} / \mathrm{THF}$, THF, $0{ }^{\circ} \mathrm{C}$ to $\mathrm{RT}$, overnight, and then (Boc) $)_{2} \mathrm{O}$, DIPEA, $\mathrm{CH}_{2} \mathrm{Cl}_{2}$, RT; for 57e: $(\mathrm{Boc})_{2} \mathrm{O}, \mathrm{NiCl}_{2} \cdot 6 \mathrm{H}_{2} \mathrm{O}, \mathrm{NaBH}_{4}, \mathrm{MeOH}, 0{ }^{\circ} \mathrm{C}, 4 \mathrm{~h}$.

\section{Scheme 2. Synthesis of Compounds 13-14 and $28^{a}$}
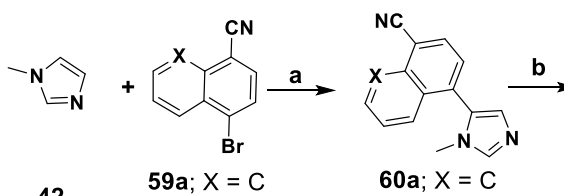

$59 b ; X=N$

$60 \mathrm{a} ; X=\mathrm{C}$
$60 \mathrm{~b} ; X=\mathrm{N}$

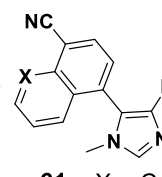

$61 \mathrm{a} ; X=\mathrm{C}$

61b; $X=N$
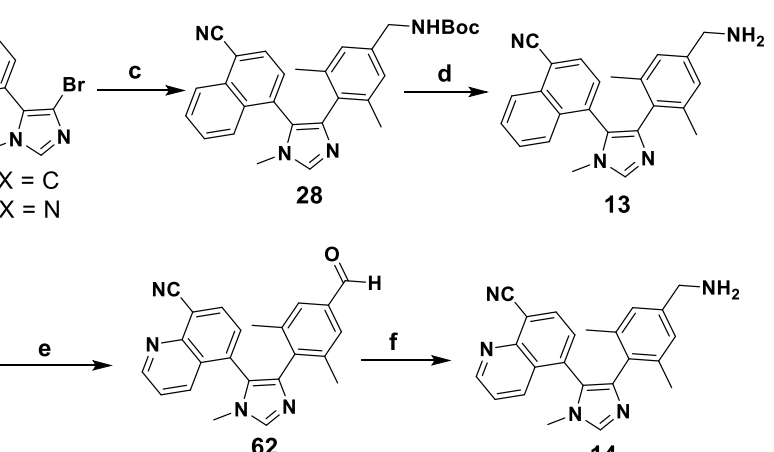

14

${ }^{a}$ Reagents and conditions: (a) $\mathrm{Pd}(\mathrm{OAc})_{2}$, AcOK, DMAc, $120{ }^{\circ} \mathrm{C}, 10 \mathrm{~h}$; (b) $\mathrm{NBS}, \mathrm{CH}_{3} \mathrm{CN}, 0{ }^{\circ} \mathrm{C}, 10$ h; (c) 58e, $\mathrm{Pd}$ (dppf) $\mathrm{Cl}_{2}, \mathrm{~K}_{2} \mathrm{CO}_{3}$, dioxane $/ \mathrm{H}_{2} \mathrm{O}, 85^{\circ} \mathrm{C}, 24 \mathrm{~h}$; (d) $4 \mathrm{M} \mathrm{HCl}$ in dioxane, $\mathrm{RT}, 1 \mathrm{~h}$; (e) 55c, $\mathrm{Pd}($ amphos $) \mathrm{Cl}_{2}, \mathrm{Cs}_{2} \mathrm{CO}_{3}$, dioxane $/ \mathrm{H}_{2} \mathrm{O}, 90^{\circ} \mathrm{C}, 24 \mathrm{~h}$; and (f) $\mathrm{CH}_{3} \mathrm{COONH}_{4}, \mathrm{CH}_{3} \mathrm{COOH}$, 
Scheme 3. Synthesis of Compounds 15 and $16^{a}$
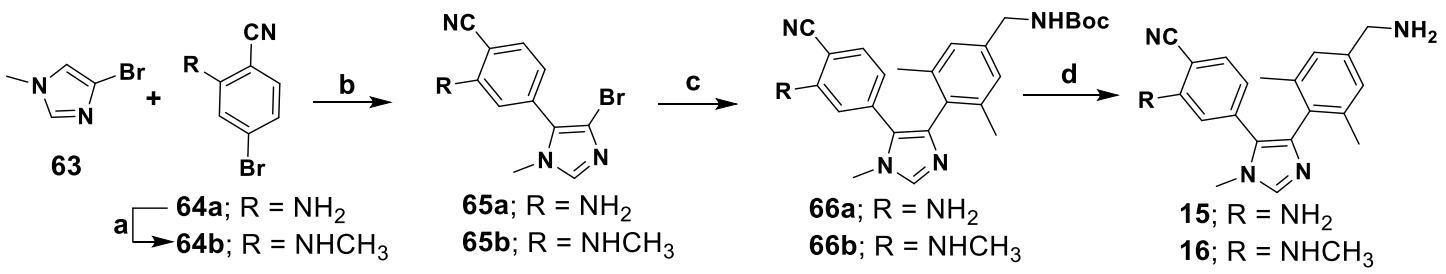

${ }^{a}$ Reagents and conditions: (a) MeI, $\mathrm{K}_{2} \mathrm{CO}_{3}$, DMF, RT, overnight; (b) $\mathrm{Pd}(\mathrm{OAc})_{2}$, AcOK, DMAc, $120{ }^{\circ} \mathrm{C}, 18 \mathrm{~h}$; (c) 58e, $\mathrm{Pd}(\operatorname{amphos}) \mathrm{Cl}_{2}, \mathrm{Cs}_{2} \mathrm{CO}_{3}$, dioxane $/ \mathrm{H}_{2} \mathrm{O}, 90^{\circ} \mathrm{C}, 24 \mathrm{~h}$; and (d) $4 \mathrm{M} \mathrm{HCl}$ in dioxane, RT, $1 \mathrm{~h}$.

Scheme 4. Synthesis of Compounds 17-20 ${ }^{a}$

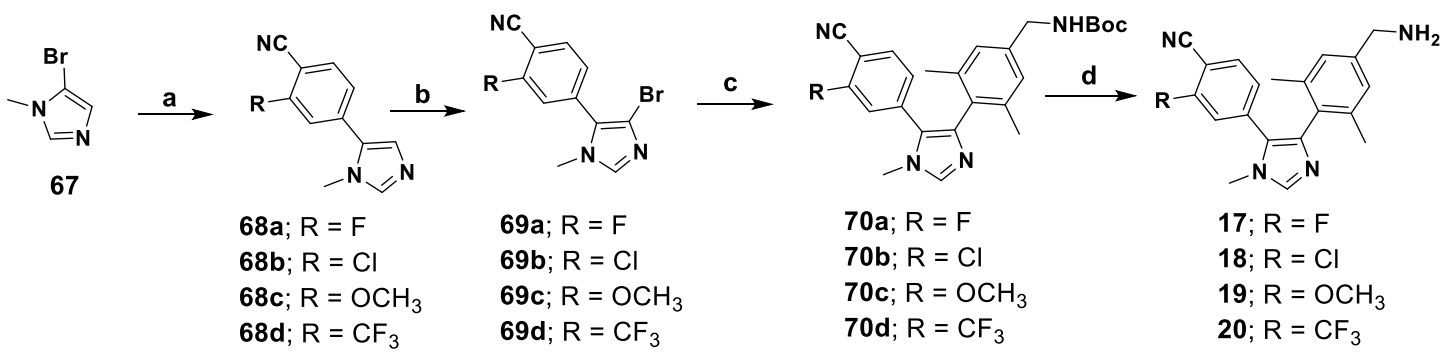

${ }^{a}$ Reagents and conditions: (a) appropriate boronic acid or boronic acid pinacol ester, $\operatorname{Pd}(\mathrm{dppf}) \mathrm{Cl}_{2}$,

$\mathrm{Na}_{2} \mathrm{CO}_{3}, \mathrm{EtOH} / \mathrm{H}_{2} \mathrm{O} /$ toluene, $100{ }^{\circ} \mathrm{C}, 10 \mathrm{~h}$; (b) $\mathrm{NBS}, \mathrm{CH}_{3} \mathrm{CN},-20{ }^{\circ} \mathrm{C}$ to RT, overnight; (c) XPhos $\mathrm{Pd} \mathrm{G} 2, \mathrm{~K}_{3} \mathrm{PO}_{4}$, dioxane $/ \mathrm{H}_{2} \mathrm{O}, 100{ }^{\circ} \mathrm{C}, 10 \mathrm{~h}$; and (d) trifluoroacetic acid, $\mathrm{CH}_{2} \mathrm{Cl}_{2}, \mathrm{RT}, 1 \mathrm{~h}$.

\section{Scheme 5. Synthesis of Compounds 21-27 ${ }^{a}$}

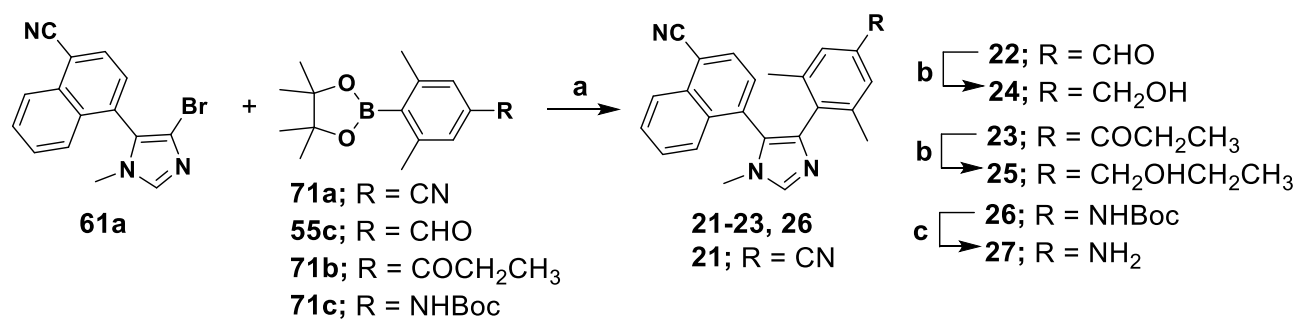

${ }^{a}$ Reagents and conditions: (a) for 22 and 26: $\mathrm{Pd}($ amphos $) \mathrm{Cl}_{2}, \mathrm{Cs}_{2} \mathrm{CO}_{3}$, dioxane $/ \mathrm{H}_{2} \mathrm{O}, 90{ }^{\circ} \mathrm{C}, 24 \mathrm{~h}$;

for 21 and 23: $\mathrm{Pd}_{2}(\mathrm{dba})_{3}, \mathrm{PCy}_{3}, \mathrm{~K}_{3} \mathrm{PO}_{4}$, toluene, $110^{\circ} \mathrm{C}$, overnight; (b) $\mathrm{NaBH}_{4}, \mathrm{MeOH}, \mathrm{RT}, 5 \mathrm{~h}$; and (c) $4 \mathrm{M} \mathrm{HCl}$ in dioxane, $\mathrm{RT}, 1 \mathrm{~h}$.

Scheme 6. Synthesis of Compounds 29-41 ${ }^{a}$ 


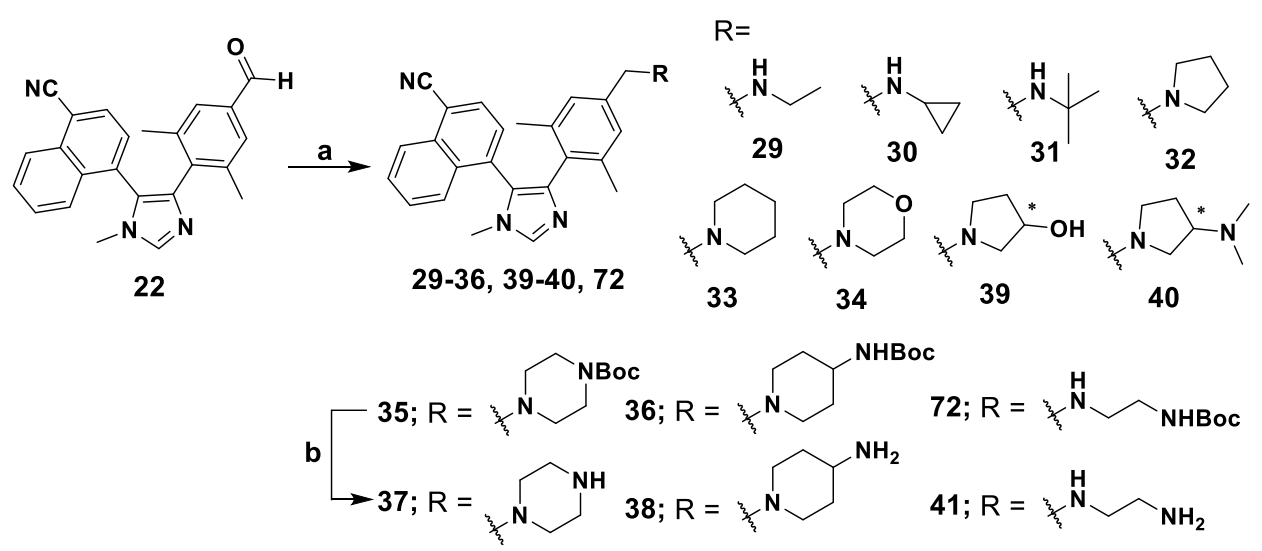

${ }^{a}$ Reagents and conditions: (a) $\mathrm{NaBH}_{3} \mathrm{CN}, \mathrm{MeOH}, 0{ }^{\circ} \mathrm{C}, 5 \mathrm{~h}$; and (b) $4 \mathrm{M} \mathrm{HCl}$ in dioxane, $\mathrm{RT}, 1 \mathrm{~h}$.

\section{CONCLUSIONS}

Based on reported NSD3-PWWP1 inhibitor BI-9321 and NSD2-PWWP1 inhibitor MR837, we discovered a selective and in vitro effective NSD2-PWWP1 inhibitor 38 via rationally design and subsequent structure-based optimization. Compound 38 exhibited good potency against NSD2-PWWP1 $\left(\mathrm{IC}_{50}=0.11 \pm 0.01 \mu \mathrm{M}\right)$ and excellent selectivity over other PWWP domains. Finally, compound $\mathbf{3 8}$ was clarified as the most potent compound, which presented quite good enzyme inhibition activity and single digit micromole level of cell proliferation inhibition activity, after our unremitting efforts on optimizing the structure of the compounds.

\section{EXPERIMENTAL SECTION}

General Information. Unless otherwise noted, all starting materials, solvents and reagents were used directly as obtained commercially. All air and moisture sensitive reactions were carried out under an atmosphere of dry argon/nitrogen with heat-dried glassware and standard syringe techniques. ${ }^{1} \mathrm{H}$ NMR and ${ }^{13} \mathrm{C}$ NMR spectra were recorded using $\mathrm{CDCl}_{3}$ or $\mathrm{CD}_{3} \mathrm{OD}$ on Varian Mercury-400, Bruker Advance $500 \mathrm{MHz}$ or $600 \mathrm{MHz}$ spectrometer with tetramethylsilane (TMS) as an internal standard. NMR 
data are reported as follows: chemical shift, integration, multiplicity (s, singlet; $d$, doublet; t, triplet; m, multiplet; dd, doublet of doublets; brs, broad singlet), and coupling constants. Low-resolution ESI-MS was obtained with Thermo Fisher Finnigan LTQ or Finnigan LCQ Deca XP mass spectrometer using a CAPCELL PAK C18 $(50 \mathrm{~mm} \times 2.0 \mathrm{~mm}, 5 \mu \mathrm{m})$ or an Agilent ZORBAX Eclipse XDB C18 $(50 \mathrm{~mm} \times$ $2.1 \mathrm{~mm}, 5 \mu \mathrm{m})$ in positive or negative electrospray mode. High-resolution ESI-MS was recorded by using Finnigan MAT-95 mass spectrometer, Agilent G6520 Q-TOF mass spectrometer or Agilent G6230 TOF LC/MS spectrometer. The target compounds were analyzed for purity by high performance liquid chromatography (HPLC) and shown to have $\geq 95 \%$ purity. Purity of target compounds was determined on analytical Gilson-215 high performance liquid chromatography system using an YMC ODS3 column $(50 \mathrm{~mm} \times 4.6 \mathrm{~mm}, 5 \mu \mathrm{m})$, and conditions were as follows: $\mathrm{CH}_{3} \mathrm{CN} / \mathrm{H}_{2} \mathrm{O}$ eluent at $2.5 \mathrm{~mL} / \mathrm{min}$ flow [containing $0.1 \%$ trifluoroacetic acid (TFA)] at $35{ }^{\circ} \mathrm{C}, 8 \mathrm{~min}$, gradient $5 \% \mathrm{CH}_{3} \mathrm{CN}$ to $95 \% \mathrm{CH}_{3} \mathrm{CN}$, monitored by $\mathrm{UV}$ absorption at 214 and $254 \mathrm{~nm}$. Purity of compounds $\mathbf{1 0}$ and $\mathbf{2 0}$ were determined by analytical Agilent-1290 high performance liquid chromatography using a Waters BEH C18 column $(50 \mathrm{~mm} \times 2.1 \mathrm{~mm}, 1.7 \mu \mathrm{m})$ and conditions were as follows: $\mathrm{CH}_{3} \mathrm{CN} / \mathrm{H}_{2} \mathrm{O}$ eluent at $0.5 \mathrm{~mL} / \mathrm{min}$ flow [containing $0.1 \%$ trifluoroacetic acid (TFA)] at $40{ }^{\circ} \mathrm{C}, 5$ min, gradient $5 \% \mathrm{CH}_{3} \mathrm{CN}$ to $80 \% \mathrm{CH}_{3} \mathrm{CN}$, monitored by $\mathrm{UV}$ absorption at 220 and $254 \mathrm{~nm}$.All reactions were monitored by TLC analysis on silica gel GF254 plates and TLC spots were visualized under UV light. Column chromatography was carried out on silica gel (200-300 mesh) for routine purification. 
Synthetic Procedures. The compound MR837 was prepared according to reported literature procedures for biological assay as positive control. ${ }^{12}$ 4-(4-(4-(aminomethyl)-2,6-dimethoxyphenyl)-1-methyl-1H-imidazol-5-yl)benzonitrile (5). To a solution of 4-Bromobenzonitrile $43(3.00 \mathrm{~g}, 16.48 \mathrm{mmol})$ and 1-methyl-1H-imidazole $42(2 \mathrm{~mL}, 32.96 \mathrm{mmol})$ in dimethylacetamide $(25 \mathrm{~mL})$ were added palladium acetate $(370 \mathrm{mg}, 1.65 \mathrm{mmol})$ and potassium acetate $(3.24 \mathrm{~g}, 32.96$ mmol). The reaction mixture was degassed with $\mathrm{Ar}_{2}$ and stirred at $120{ }^{\circ} \mathrm{C}$ for $10 \mathrm{~h}$. After cooling to rt, the reaction was quenched by addition of saturated aqueous $\mathrm{NaCl}$ $(100 \mathrm{~mL})$ and was extracted with EtOAc $(3 \times 20 \mathrm{~mL})$. The combined organic phase was washed with brine $(50 \mathrm{~mL} \times 5)$ and dried over anhydrous $\mathrm{Na}_{2} \mathrm{SO}_{4}$. The residue was purified by column chromatography on silica gel $\left(4: 1,60-90{ }^{\circ} \mathrm{C}\right.$ petroleum ether EtOAc) to give the desired intermediate (44). Off-white solid (1.46 g, $48.3 \%) .{ }^{1} \mathrm{H}$ NMR (400 MHz, Methanol- $\left.d_{4}\right) \delta 7.82-7.77(\mathrm{~m}, 2 \mathrm{H}), 7.76(\mathrm{~d}, \mathrm{~J}=1.1 \mathrm{~Hz}, 1 \mathrm{H}), 7.70-$ $7.63(\mathrm{~m}, 2 \mathrm{H}), 7.20(\mathrm{~d}, \mathrm{~J}=1.1 \mathrm{~Hz}, 1 \mathrm{H}), 3.77(\mathrm{~s}, 3 \mathrm{H})$.

To a solution of compound (44) $(970 \mathrm{mg}, 5.29 \mathrm{mmol})$ in $\mathrm{CH}_{3} \mathrm{CN}(20 \mathrm{~mL})$ under ice bath was added NBS (940 mg, $5.28 \mathrm{mmol}$ ) in three batches. The ice bath was removed and the mixture was stirred at $\mathrm{rt}$ for $10 \mathrm{~h}$. The reaction was slowly quenched by saturated aqueous $\mathrm{Na}_{2} \mathrm{~S}_{2} \mathrm{O}_{3}$ and was extracted with EtOAc $(3 \times 10 \mathrm{~mL})$. The combined organic phase was washed with brine $(10 \mathrm{~mL})$ and dried over anhydrous $\mathrm{Na}_{2} \mathrm{SO}_{4}$, filtered, and concentrated in vacuo. The residue was purified by column chromatography on silica gel $\left(6: 1,60-90{ }^{\circ} \mathrm{C}\right.$ petroleum ether - EtOAc) to give the intermediate (45). White solid (668 mg, 48.1\%). ${ }^{1} \mathrm{H}$ NMR (400 MHz, Methanol- $\left.d_{4}\right) \delta$ 
$7.90-7.86(\mathrm{~m}, 2 \mathrm{H}), 7.77(\mathrm{~s}, 1 \mathrm{H}), 7.70-7.66(\mathrm{~m}, 2 \mathrm{H}), 3.67$ (s, 3H).

Compound 45 (145 mg, $554.53 \mu \mathrm{mol})$, 55a (180 mg, $616.15 \mu \mathrm{mol})$, and $\mathrm{K}_{2} \mathrm{CO}_{3}$ (170 mg, $1.23 \mathrm{mmol}$ ) were dissolved in 1, 4-dioxane (4.8 mL) and $\mathrm{H}_{2} \mathrm{O}(1.2 \mathrm{~mL})$. The mixture was degassed with $\mathrm{Ar}_{2}$ and $\mathrm{PdCl}_{2}(\mathrm{dppf})(50 \mathrm{mg}, 61.61 \mu \mathrm{mol})$ was added quickly under argon atmosphere. The reaction mixture was heated to $100{ }^{\circ} \mathrm{C}$ and stirred for $7 \mathrm{~h}$. After completion of the reaction, the mixture was concentrated under reduced pressure and purified by column chromatography on silica gel (5:1, 60 - 90 ${ }^{\circ} \mathrm{C}$ petroleum ether $-\mathrm{EtOAc}$ ) to give the intermediate (46). White solid (74 $\mathrm{mg}$, $34.6 \%) .{ }^{1} \mathrm{H}$ NMR (400 MHz, Methanol- $\left.d_{4}\right) \delta 9.92(\mathrm{~d}, \mathrm{~J}=0.9 \mathrm{~Hz}, 1 \mathrm{H}), 7.85(\mathrm{~s}, 1 \mathrm{H})$, $7.71-7.65(\mathrm{~m}, 2 \mathrm{H}), 7.44-7.38(\mathrm{~m}, 2 \mathrm{H}), 7.17(\mathrm{~s}, 2 \mathrm{H}), 3.74(\mathrm{~s}, 3 \mathrm{H}), 3.67(\mathrm{~s}, 6 \mathrm{H})$.

To a solution of $46(50 \mathrm{mg}, 143.94 \mu \mathrm{mol})$ and ammonium acetate $(111 \mathrm{mg}, 1.44$ mmol) in $\mathrm{CH}_{3} \mathrm{OH}(4 \mathrm{~mL})$ was added a drop of $\mathrm{CH}_{3} \mathrm{COOH}$ and the resulting mixture was stirred at room temperature for $0.5 \mathrm{~h}$. Sodium cyanoborohydride (46 mg, 215.91 $\mu \mathrm{mol})$ was added into the mixture at $0{ }^{\circ} \mathrm{C}$ and the reaction mixture was then stirred for $8 \mathrm{~h}$. Subsequently, the reaction was quenched by saturated aqueous $\mathrm{NaHCO}_{3}$, and was extracted with DCM $-\mathrm{MeOH}(5: 1,3 \times 5 \mathrm{~mL})$. The combined organic phase was washed with brine $(10 \mathrm{~mL})$ and dried over anhydrous $\mathrm{Na}_{2} \mathrm{SO}_{4}$, filtered, and concentrated in vacuo. The residue was purified by column chromatography on silica gel (20:1, DCM - MeOH) to obtain the desired product (5). Off-white amorphous solid (668 mg, $48.1 \%) .{ }^{1} \mathrm{H}$ NMR (600 MHz, Methanol- $\left.d_{4}\right) \delta 7.79(\mathrm{~s}, 1 \mathrm{H}), 7.67-7.58$ (m, 2H), $7.44-7.37(\mathrm{~m}, 2 \mathrm{H}), 6.62(\mathrm{~s}, 2 \mathrm{H}), 3.79(\mathrm{~s}, 2 \mathrm{H}), 3.71(\mathrm{~s}, 3 \mathrm{H}), 3.60(\mathrm{~s}, 6 \mathrm{H}) .{ }^{13} \mathrm{C}$ NMR (151 MHz, Methanol- $\left.d_{4}\right) \delta 160.54(* 2 \mathrm{C}), 145.91,140.02,136.66,134.28$, 
$133.15(* 2 \mathrm{C}), 130.92,130.84(* 2 \mathrm{C}), 119.56,112.01,111.43,104.20(* 2 \mathrm{C}), 56.04(* 2 \mathrm{C})$, 46.96, 33.26. HRMS (ESI): $m / z[\mathrm{M}+\mathrm{H}]^{+} \mathrm{C}_{20} \mathrm{H}_{21} \mathrm{~N}_{4} \mathrm{O}_{2}$ 349.1659; found 349.1661. HPLC: $t_{\mathrm{R}}=2.000 \min , 100 \%$.

\section{4-(4-(4-(aminomethyl)-2-methoxyphenyl)-1-methyl-1H-imidazol-5-yl)benzonitrile}

(6). The intermediate 47 was obtained from 45 through a procedure similar to the preparation of the intermediate 46. Off-white solid. 71.4\% yield. ${ }^{1} \mathrm{H}$ NMR (400 MHz, Methanol- $\left.d_{4}\right) \delta 9.93(\mathrm{~s}, 1 \mathrm{H}), 7.88(\mathrm{~s}, 1 \mathrm{H}), 7.75(\mathrm{~d}, J=0.6 \mathrm{~Hz}, 1 \mathrm{H}), 7.73(\mathrm{~d}, J=0.6 \mathrm{~Hz}$, 1H), $7.71-7.66(\mathrm{~m}, 1 \mathrm{H}), 7.54(\mathrm{dd}, J=7.7,1.5 \mathrm{~Hz}, 1 \mathrm{H}), 7.42(\mathrm{~d}, J=0.6 \mathrm{~Hz}, 1 \mathrm{H}), 7.40$ (q, $J=1.5 \mathrm{~Hz}, 1 \mathrm{H}), 7.34(\mathrm{~d}, J=1.4 \mathrm{~Hz}, 1 \mathrm{H}), 3.71(\mathrm{~s}, 3 \mathrm{H}), 3.33(\mathrm{~s}, 3 \mathrm{H})$.

The target compound $\mathbf{6}$ was prepared from $\mathbf{4 7}$ by following a similar method as described for compound 5. Off-white amorphous solid. 52.3\% yield. ${ }^{1} \mathrm{H}$ NMR (600 MHz, Methanol- $\left.d_{4}\right) \delta 7.81(\mathrm{~s}, 1 \mathrm{H}), 7.72-7.69(\mathrm{~m}, 2 \mathrm{H}), 7.41-7.38(\mathrm{~m}, 2 \mathrm{H}), 7.36(\mathrm{~d}$, $\mathrm{J}=7.7 \mathrm{~Hz}, 1 \mathrm{H}), 6.94(\mathrm{~d}, \mathrm{~J}=7.7 \mathrm{~Hz}, 1 \mathrm{H}), 6.88(\mathrm{~s}, 1 \mathrm{H}), 3.79(\mathrm{~s}, 2 \mathrm{H}), 3.70(\mathrm{~s}, 3 \mathrm{H}), 3.34$ (s, 3H). ${ }^{13} \mathrm{C}$ NMR (151 MHz, Methanol- $\left.d_{4}\right) \delta 157.88,144.88,140.04,137.64,137.39$, 133.25(*2C), 132.36, 131.16(*2C), 130.17, 122.98, 120.58, 119.55, 112.19, 111.36, 55.03, 46.54, 33.27. HRMS (ESI): $m / z[\mathrm{M}+\mathrm{H}]^{+}$calcd for $\mathrm{C}_{19} \mathrm{H}_{19} \mathrm{~N}_{4} \mathrm{O}$ 319.1553; found 319.1548. HPLC: $t_{\mathrm{R}}=1.932 \mathrm{~min}, 96.54 \%$.

4-(4-(4-(aminomethyl)-2,6-dimethylphenyl)-1-methyl-1H-imidazol-5-yl)benzonitrile (12). The intermediate 48 was obtained from 45 through a procedure similar to the preparation of the intermediate 46. Orange solid. $12.4 \%$ yield. ${ }^{1} \mathrm{H}$ NMR (400 MHz, Methanol- $\left.d_{4}\right) \delta 9.91(\mathrm{~s}, 1 \mathrm{H}), 7.98(\mathrm{~s}, 1 \mathrm{H}), 7.68(\mathrm{~d}, \mathrm{~J}=8.3 \mathrm{~Hz}, 2 \mathrm{H}), 7.58(\mathrm{~s}, 2 \mathrm{H}), 7.35$ $(\mathrm{dd}, \mathrm{J}=7.4,1.3 \mathrm{~Hz}, 2 \mathrm{H}), 3.81(\mathrm{~s}, 3 \mathrm{H}), 2.09(\mathrm{~s}, 6 \mathrm{H})$. 
The target compound $\mathbf{1 2}$ was prepared from $\mathbf{4 8}$ by following a similar method as described for compound 5. Off-white amorphous solid. 58.3\% yield. ${ }^{1}$ H NMR (400 MHz, Methanol- $\left.d_{4}\right) \delta 9.28(\mathrm{~s}, 1 \mathrm{H}), 7.81(\mathrm{~d}, \mathrm{~J}=8.0 \mathrm{~Hz}, 2 \mathrm{H}), 7.56(\mathrm{~d}, \mathrm{~J}=8.1 \mathrm{~Hz}, 2 \mathrm{H})$, $7.34(\mathrm{~s}, 2 \mathrm{H}), 4.22(\mathrm{~s}, 2 \mathrm{H}), 3.97$ (s, 3H), 2.15 (s, 6H). ${ }^{13} \mathrm{C}$ NMR (151 MHz, Methanol- $\left.d_{4}\right) \delta 141.27(* 2 \mathrm{C}), 138.47,134.75,134.05(* 2 \mathrm{C}), 131.96,131.74(* 2 \mathrm{C})$, 131.66, 131.34, 130.57(*2C), 127.37, 118.76, 115.00, 51.71, 35.43, 20.34(*2C). HRMS (ESI): $m / z[\mathrm{M}+\mathrm{H}]^{+}$calcd for $\mathrm{C}_{20} \mathrm{H}_{21} \mathrm{~N}_{4}$ 317.1761; found 317.1757. HPLC: $t_{\mathrm{R}}$ $=2.383 \min , 96.77 \%$.

4-(4-(4-(aminomethyl)phenyl)-1-methyl-1H-imidazol-5-yl)benzonitrile (7). Compound 45 (47.4 mg, $181 \mu \mathrm{mol})$, (4-(((tert-butoxycarbonyl)amino)methyl)phenyl)boronic acid (50 mg, $199 \mu \mathrm{mol})$, and $\mathrm{K}_{3} \mathrm{PO}_{4}(170 \mathrm{mg}, 1.23 \mathrm{mmol})$ were dissolved in 1, 4-dioxane (4.8 mL) and $\mathrm{H}_{2} \mathrm{O}(1.2 \mathrm{~mL})$. The mixture was degassed with $\mathrm{Ar}_{2}$ and XPhos Pd G2 (14.2 mg, $18.1 \mu \mathrm{mol})$ was added quickly under argon atmosphere. The reaction mixture was heated to $90{ }^{\circ} \mathrm{C}$ and stirred overnight. After completion of the reaction, the mixture was concentrated under reduced pressure and purified by column chromatography on silica gel $\left(5: 1,60-90{ }^{\circ} \mathrm{C}\right.$ petroleum ether - EtOAc) to give the intermediate (49) as a white solid. To a stirred solution of 49 in DCM (2 ml) at $0{ }^{\circ} \mathrm{C}$ was added TFA $(1 \mathrm{ml})$ and the mixture stirred at $\mathrm{rt}$ for $1 \mathrm{~h}$. The mixture was concentrated, and the residue was purified by column chromatography on silica gel (20:1, DCM - MeOH) to give compound 7. White amorphous solid (12 mg, $23 \%$ yield over two steps). ${ }^{1} \mathrm{H}$ NMR $\left(600 \mathrm{MHz}\right.$, Methanol- $\left.d_{4}\right) \delta 7.82-7.81(\mathrm{~m}, 2 \mathrm{H}), 7.80$ $(\mathrm{d}, \mathrm{J}=1.9 \mathrm{~Hz}, 1 \mathrm{H}), 7.54-7.51(\mathrm{~m}, 2 \mathrm{H}), 7.31-7.28(\mathrm{~m}, 2 \mathrm{H}), 7.22(\mathrm{~d}, \mathrm{~J}=8.4 \mathrm{~Hz}, 2 \mathrm{H})$, 
$3.75(\mathrm{~s}, 2 \mathrm{H}), 3.61(\mathrm{~s}, 3 \mathrm{H}) .{ }^{13} \mathrm{C}$ NMR (151 MHz, Methanol-d $\left.)_{4}\right) \delta$ 142.29, 140.23, $140.17, \quad 136.50, \quad 133.86(* 2 \mathrm{C}), \quad 133.79, \quad 132.72(* 2 \mathrm{C}), \quad 130.88, \quad 128.71(* 2 \mathrm{C})$, 128.56(*2C), 119.37, 113.32, 46.24, 32.92. HRMS (ESI): $m / z[M+H]^{+}$calcd for $\mathrm{C}_{18} \mathrm{H}_{17} \mathrm{~N}_{4}$ 289.1448; found 289.1446. HPLC: $t_{\mathrm{R}}=1.854 \mathrm{~min}, 96.01 \%$. 4-(4-(4-(aminomethyl)-2-chlorophenyl)-1-methyl-1H-imidazol-5-yl)benzonitrile (8). The target compound $\mathbf{8}$ was prepared from $\mathbf{4 5}$ and $\mathbf{5 8 a}$ by following a similar method as described for compound 7. White amorphous solid (18 mg, $30 \%$ yield over two steps). ${ }^{1} \mathrm{H}$ NMR $\left(600 \mathrm{MHz}\right.$, Methanol- $\left.d_{4}\right) \delta 7.86(\mathrm{~s}, 1 \mathrm{H}), 7.71-7.67(\mathrm{~m}, 2 \mathrm{H}), 7.43-$ $7.39(\mathrm{~m}, 2 \mathrm{H}), 7.38(\mathrm{~d}, \mathrm{~J}=1.4 \mathrm{~Hz}, 1 \mathrm{H}), 7.33(\mathrm{~d}, \mathrm{~J}=7.8 \mathrm{~Hz}, 1 \mathrm{H}), 7.27(\mathrm{dd}, \mathrm{J}=7.9,1.7$ $\mathrm{Hz}, 1 \mathrm{H}), 3.78(\mathrm{~s}, 2 \mathrm{H}), 3.74(\mathrm{~s}, 3 \mathrm{H}) .{ }^{13} \mathrm{C}$ NMR (151 MHz, Methanol- $\left.d_{4}\right) \delta 145.79$, $140.32,138.64,135.92,135.01,133.70,133.51(* 2 \mathrm{C}), 132.85,131.50(* 2 \mathrm{C}), 130.61$, 129.64, 127.01, 119.38, 112.70, 45.84, 33.34. HRMS (ESI): $m / z[\mathrm{M}+\mathrm{H}]^{+}$calcd for $\mathrm{C}_{18} \mathrm{H}_{16} \mathrm{ClN}_{4}$ 323.1058; found 307.1053. HPLC: $t_{\mathrm{R}}=2.233 \mathrm{~min}, 100 \%$.

4-(4-(4-(aminomethyl)-2-fluorophenyl)-1-methyl-1H-imidazol-5-yl)benzonitrile (9).

The target compound 9 was prepared from $\mathbf{4 5}$ and $\mathbf{5 8 b}$ by following a similar method as described for compound 7. White amorphous solid (12 mg, $21 \%$ yield over two steps). ${ }^{1} \mathrm{H}$ NMR (500 MHz, Methanol- $\left.d_{4}\right) \delta 7.87(\mathrm{~s}, 1 \mathrm{H}), 7.75-7.69(\mathrm{~m}, 2 \mathrm{H}), 7.45$ (dt, $\mathrm{J}=8.2,1.6 \mathrm{~Hz}, 2 \mathrm{H}), 7.41(\mathrm{t}, \mathrm{J}=7.7 \mathrm{~Hz}, 1 \mathrm{H}), 7.15(\mathrm{dd}, \mathrm{J}=7.9,1.6 \mathrm{~Hz}, 1 \mathrm{H}), 7.01(\mathrm{dd}$, $\mathrm{J}=11.2,1.4 \mathrm{~Hz}, 1 \mathrm{H}), 3.79(\mathrm{~s}, 2 \mathrm{H}), 3.69(\mathrm{~s}, 3 \mathrm{H}) .{ }^{13} \mathrm{C}$ NMR $\left(126 \mathrm{MHz}\right.$, Methanol- $\left.d_{4}\right) \delta$ 140.64, 136.19, 133.52(*2C), 132.65, 132.62, 131.66(*2C), 130.88, 130.80, 130.73, 124.33, 119.41, 115.78, 115.60, 112.88, 45.82, 33.26. HRMS (ESI): $m / z[M+H]^{+}$ calcd for $\mathrm{C}_{18} \mathrm{H}_{16} \mathrm{~N}_{4}$ 307.1354; found 307.1352. HPLC: $t_{\mathrm{R}}=1.864$ min, $100 \%$. 
nitrile (10). The target compound 10 was prepared from 45 and $58 \mathrm{c}$ by following a similar method as described for compound 7. White amorphous solid (12 mg, $18 \%$ yield over two steps). ${ }^{1} \mathrm{H}$ NMR (500 MHz, MeOD- $\left.d_{4}\right): \delta 8.86(\mathrm{~s}, 1 \mathrm{H}), 7.99(\mathrm{~d}, J=0.9$ $\mathrm{Hz}, 1 \mathrm{H}), 7.79(\mathrm{~d}, J=8.5 \mathrm{~Hz}, 2 \mathrm{H}), 7.65(\mathrm{~d}, J=7.9 \mathrm{~Hz}, 1 \mathrm{H}), 7.55(\mathrm{~d}, J=8.5 \mathrm{~Hz}, 2 \mathrm{H})$, $4.27(\mathrm{~s}, 2 \mathrm{H}), 3.86(\mathrm{~s}, 3 \mathrm{H})$. LRMS (ESI): $\mathrm{m} / z[\mathrm{M}+\mathrm{H}]^{+}$calcd for $\mathrm{C}_{19} \mathrm{H}_{16} \mathrm{~F}_{3} \mathrm{~N}_{4}$ 357.1; found 357.3. HPLC: $t_{\mathrm{R}}=3.814 \mathrm{~min}, 99.01 \%$.

\section{4-(4-(4-(aminomethyl)-2-methylphenyl)-1-methyl-1H-imidazol-5-yl)benzonitrile}

(11). The target compound $\mathbf{1 1}$ was prepared from $\mathbf{4 5}$ and $\mathbf{5 8 d}$ by following a similar method as described for compound 7. White amorphous solid (17 mg, $32 \%$ yield over two steps). ${ }^{1} \mathrm{H}$ NMR (600 MHz, Methanol- $\left.d_{4}\right) \delta 7.85(\mathrm{~s}, 1 \mathrm{H}), 7.67(\mathrm{~d}, \mathrm{~J}=8.3 \mathrm{~Hz}, 2 \mathrm{H})$, $7.42-7.32(\mathrm{~m}, 2 \mathrm{H}), 7.17(\mathrm{~s}, 1 \mathrm{H}), 7.10(\mathrm{~s}, 2 \mathrm{H}), 3.74(\mathrm{~s}, 2 \mathrm{H}), 3.74(\mathrm{~s}, 3 \mathrm{H}), 2.05(\mathrm{~s}, 3 \mathrm{H})$. ${ }^{13} \mathrm{C}$ NMR (151 MHz, Methanol- $\left.d_{4}\right) \delta 143.43,141.07,140.27,138.57,136.06$, $133.48(* 2 \mathrm{C}), 132.19,131.56(* 2 \mathrm{C}), 130.39,129.77,125.75,119.40,112.43,46.32$, 33.37, 20.22. HRMS (ESI): $m / z[M+H]^{+}$calcd for $\mathrm{C}_{19} \mathrm{H}_{19} \mathrm{~N}_{4}$ 303.1604; found 303.1609. HPLC: $t_{\mathrm{R}}=1.917 \mathrm{~min}, 95.59 \%$.

tert-butyl

(4-(5-(4-cyanonaphthalen-1-yl)-1-methyl-1H-imidazol-4-yl)-3,5-dimethylbenzyl)carba mate (28). The intermediate 60a was obtained from 42 and 59a through a procedure similar to the preparation of the intermediate 44. Light green amorphous solid. $63.4 \%$ yield. ${ }^{1} \mathrm{H}$ NMR (400 MHz, Methanol- $\left.d_{4}\right) \delta 8.32-8.25(\mathrm{~m}, 1 \mathrm{H}), 8.10(\mathrm{~d}, J=7.4 \mathrm{~Hz}$, 1H), $7.90(\mathrm{~d}, J=1.1 \mathrm{~Hz}, 1 \mathrm{H}), 7.84-7.78(\mathrm{~m}, 2 \mathrm{H}), 7.71(\mathrm{ddd}, J=8.0,6.9,1.3 \mathrm{~Hz}, 1 \mathrm{H})$, 
$7.64(\mathrm{~d}, J=7.4 \mathrm{~Hz}, 1 \mathrm{H}), 7.16(\mathrm{~d}, J=1.2 \mathrm{~Hz}, 1 \mathrm{H}), 3.49$ (s, 3H).

The intermediate 61a was obtained from 60a through a procedure similar to the preparation of the intermediate 45 . White solid. $32.4 \%$ yield. ${ }^{1} \mathrm{H}$ NMR (400 MHz, Methanol- $\left.d_{4}\right) \delta 8.32(\mathrm{dt}, J=8.4,1.0 \mathrm{~Hz}, 1 \mathrm{H}), 8.15(\mathrm{~d}, J=7.4 \mathrm{~Hz}, 1 \mathrm{H}), 7.89(\mathrm{~s}, 1 \mathrm{H})$, $7.84(\mathrm{ddd}, J=8.3,6.9,1.3 \mathrm{~Hz}, 1 \mathrm{H}), 7.73(\mathrm{ddd}, J=8.2,6.8,1.2 \mathrm{~Hz}, 1 \mathrm{H}), 7.67$ (d, $J=$ $7.4 \mathrm{~Hz}, 1 \mathrm{H}), 7.64(\mathrm{dt}, J=8.5,1.0 \mathrm{~Hz}, 1 \mathrm{H}), 3.46(\mathrm{~s}, 3 \mathrm{H})$.

The target compound 28 was prepared from 61a and 58e by following a similar method as described for compound 46. Yellow amorphous solid (21 mg, $14.2 \%$ yield). ${ }^{1} \mathrm{H}$ NMR 1H NMR (400 MHz, Chloroform- $d$ ) $\delta 8.28(\mathrm{~d}, \mathrm{~J}=8.2 \mathrm{~Hz}, 1 \mathrm{H}), 7.81(\mathrm{~s}, 1 \mathrm{H})$, $7.80(\mathrm{~d}, \mathrm{~J}=7.4 \mathrm{~Hz}, 1 \mathrm{H}), 7.76-7.67(\mathrm{~m}, 2 \mathrm{H}), 7.66-7.56(\mathrm{~m}, 1 \mathrm{H}), 7.24(\mathrm{~d}, \mathrm{~J}=7.4 \mathrm{~Hz}$ 1H), $6.90(\mathrm{~s}, 1 \mathrm{H}), 6.70(\mathrm{~s}, 1 \mathrm{H}), 4.14(\mathrm{~d}, \mathrm{~J}=5.2 \mathrm{~Hz}, 2 \mathrm{H}), 3.43(\mathrm{~s}, 3 \mathrm{H}), 2.22(\mathrm{~s}, 3 \mathrm{H})$, $1.79(\mathrm{~s}, 3 \mathrm{H}), 1.42(\mathrm{~s}, 9 \mathrm{H})$. HRMS (ESI): $m / z[\mathrm{M}+\mathrm{H}]^{+}$calcd for $\mathrm{C}_{29} \mathrm{H}_{31} \mathrm{~N}_{4} \mathrm{O}_{2}$ 467.2442; found 467.2437. HPLC: $t_{\mathrm{R}}=3.205 \mathrm{~min}, 100 \%$.

\section{4-(4-(4-(aminomethyl)-2,6-dimethylphenyl)-1-methyl-1H-imidazol-5-yl)-1-naphtho}

nitrile (13). The compound $28(10 \mathrm{mg}, 21.4 \mu \mathrm{mol})$ was dissolved in $4 \mathrm{M} \mathrm{HCl}$ in 1,4-dioxane solution $(2 \mathrm{~mL})$ at room temperature and stirred for $1 \mathrm{~h}$. The solvent was removed in vacuo, neutralized with ammonia alcohol solution and purified by column chromatography on silica gel $(20: 1, \mathrm{DCM}-\mathrm{MeOH})$ to obtain the desired product (13). Yellow amorphous solid (6 mg, 82.3\%). ${ }^{1} \mathrm{H}$ NMR (400 MHz, Methanol- $\left.d_{4}\right) \delta 9.40(\mathrm{~s}$, 1H), $8.30(\mathrm{~d}, \mathrm{~J}=8.2 \mathrm{~Hz}, 1 \mathrm{H}), 8.05(\mathrm{~d}, \mathrm{~J}=6.7 \mathrm{~Hz}, 1 \mathrm{H}), 7.95(\mathrm{~d}, \mathrm{~J}=7.3 \mathrm{~Hz}, 1 \mathrm{H}), 7.91-$ $7.79(\mathrm{~m}, 2 \mathrm{H}), 7.65(\mathrm{~d}, \mathrm{~J}=6.8 \mathrm{~Hz}, 1 \mathrm{H}), 7.26(\mathrm{~s}, 1 \mathrm{H}), 7.10(\mathrm{~s}, 1 \mathrm{H}), 4.01(\mathrm{~s}, 2 \mathrm{H}), 3.73(\mathrm{~s}$, 3H), $2.35(\mathrm{~s}, 3 \mathrm{H}), 2.05(\mathrm{~s}, 3 \mathrm{H}) .{ }^{13} \mathrm{C}$ NMR (151 MHz, Methanol- $\left.d_{4}\right) \delta$ 141.63, 141.10, 
$138.58,136.82,133.67,133.28,132.82,131.18,131.04,130.96,130.88,130.67$, $129.44,129.35,129.27,127.26,126.91(* 2 \mathrm{C}), 117.60,114.22,43.69,35.56,20.80$, 20.72.ESI $\left([\mathrm{M}+\mathrm{H}]^{+}\right) \mathrm{m} / z: 367.27$. HRMS (ESI): $\mathrm{m} / z[\mathrm{M}+\mathrm{H}]^{+}$calcd for $\mathrm{C}_{24} \mathrm{H}_{23} \mathrm{~N}_{4}$ 367.1917; found 367.1910. HPLC: $t_{\mathrm{R}}=2.268 \mathrm{~min}, 97.79 \%$.

\section{5-(4-(4-(aminomethyl)-2,6-dimethylphenyl)-1-methyl-1H-imidazol-5-yl)quinoline-8}

-carbonitrile (14). The intermediate $60 \mathrm{~b}$ was obtained from 42 and $59 \mathrm{~b}$ through a procedure similar to the preparation of the intermediate 60a. Light brown solid. $42.5 \%$ yield. ${ }^{1} \mathrm{H}$ NMR (400 MHz, Chloroform- $d$ ) $\delta 9.21-9.10(\mathrm{~m}, 1 \mathrm{H}), 8.32-8.12(\mathrm{~m}, 2 \mathrm{H})$, $7.72(\mathrm{~s}, 1 \mathrm{H}), 7.64-7.50(\mathrm{~m}, 2 \mathrm{H}), 7.23(\mathrm{~s}, 1 \mathrm{H}), 3.52(\mathrm{~s}, 3 \mathrm{H})$.

The intermediate $61 \mathbf{b}$ was obtained from $60 \mathrm{~b}$ through a procedure similar to the preparation of the intermediate 61a. Light yellow solid. $89.1 \%$ yield. ${ }^{1} \mathrm{H}$ NMR (400 MHz, Chloroform- $d) \delta 9.17(\mathrm{~d}, J=3.9 \mathrm{~Hz}, 1 \mathrm{H}), 8.24(\mathrm{~d}, J=7.3 \mathrm{~Hz}, 2 \mathrm{H}), 8.01(\mathrm{~d}, J=$ $8.3 \mathrm{~Hz}, 1 \mathrm{H}), 7.73-7.52(\mathrm{~m}, 2 \mathrm{H}), 3.47(\mathrm{~s}, 3 \mathrm{H})$.

Compound 61b (77 mg, $294.7 \mu \mathrm{mol}), \mathbf{5 5 c}(92 \mathrm{mg}, 294.7 \mu \mathrm{mol})$, and $\mathrm{Cs}_{2} \mathrm{CO}_{3}(288$ $\mathrm{mg}, 884.1 \mu \mathrm{mol})$ were dissolved in 1, 4-dioxane $(4.8 \mathrm{~mL})$ and $\mathrm{H}_{2} \mathrm{O}(1.2 \mathrm{~mL})$. The mixture was degassed with $\mathrm{Ar}_{2}$ and $\mathrm{Pd}($ amphos $) \mathrm{Cl}_{2}(21 \mathrm{mg}, 29.47 \mu \mathrm{mol})$ was added quickly under argon atmosphere. The reaction mixture was heated to $90{ }^{\circ} \mathrm{C}$ and stirred for $24 \mathrm{~h}$. After completion of the reaction, the mixture was concentrated under reduced pressure and purified by column chromatography on silica gel (4:1, 60 - 90 ${ }^{\circ} \mathrm{C}$ petroleum ether - EtOAc) to give the intermediate 62. Light yellow solid (20 mg, 22.4\%). ${ }^{1} \mathrm{H}$ NMR (400 MHz, Chloroform-d) $\delta 9.85$ (s, 1H), $9.11(\mathrm{dd}, J=4.1,1.4 \mathrm{~Hz}$, $1 \mathrm{H}), 8.05(\mathrm{~d}, J=7.4 \mathrm{~Hz}, 1 \mathrm{H}), 8.03-7.98(\mathrm{~m}, 1 \mathrm{H}), 7.90(\mathrm{~s}, 1 \mathrm{H}), 7.53(\mathrm{dd}, J=8.5,4.2$ 
Hz, 1H), 7.48 (s, 1H), 7.38 (s, 1H), 7.34 (d, J= 7.5 Hz, 1H), 3.51 (s, 3H), 2.22 (s, 3H), $1.97(\mathrm{~s}, 3 \mathrm{H})$.

The target compound 14 was prepared from 62 by following a similar method as described for compound 5. Light yellow amorphous solid (8 mg, 42.0\%). ${ }^{1} \mathrm{H}$ NMR $\left(500 \mathrm{MHz}\right.$, Methanol- $\left.d_{4}\right) \delta 9.10-9.05(\mathrm{~m}, 2 \mathrm{H}), 8.32(\mathrm{ddd}, \mathrm{J}=8.5,4.4,1.4 \mathrm{~Hz}, 1 \mathrm{H})$, $8.26(\mathrm{~d}, \mathrm{~J}=7.5 \mathrm{~Hz}, 1 \mathrm{H}), 7.79-7.68(\mathrm{~m}, 2 \mathrm{H}), 7.21(\mathrm{~d}, \mathrm{~J}=14.2 \mathrm{~Hz}, 1 \mathrm{H}), 7.11(\mathrm{~d}, \mathrm{~J}=$ $17.5 \mathrm{~Hz}, 1 \mathrm{H}), 3.99$ (s, 2H), $3.71(\mathrm{~s}, 3 \mathrm{H}), 2.26$ (s, 3H), 2.06 (s, 3H). ${ }^{13} \mathrm{C}$ NMR (126 MHz, Methanol- $\left.d_{4}\right) \delta 154.33,148.39,141.41,140.76,139.41,136.50,136.27,135.47$, $131.40,131.36,130.72,130.11,129.32,129.26,128.70,125.24,125.21,117.23$, 116.16, 43.72, 34.97, 20.71, 20.68. HRMS (ESI): $m / z$. $[\mathrm{M}+\mathrm{H}]^{+}$calcd for $\mathrm{C}_{23} \mathrm{H}_{22} \mathrm{~N}_{5}$ 368.1870; found 322.1872. HPLC: $t_{\mathrm{R}}=2.712 \mathrm{~min}, 100 \%$.

\section{2-amino-4-(4-(4-(aminomethyl)-2,6-dimethylphenyl)-1-methyl-1H-imidazol-5-yl)be}

nzonitrile (15). To a solution of 2-amino-4-bromobenzonitrile 64a (500 mg, 2.54 mmol) and 4-bromo-1-methyl-1H-imidazole $42(310 \mu \mathrm{L}, \quad 3.05 \quad \mathrm{mmol})$ in dimethylacetamide $(10 \mathrm{~mL})$ were added palladium acetate $(60 \mathrm{mg}, 254 \mu \mathrm{mol})$ and potassium acetate $(500 \mathrm{mg}, 5.10 \mathrm{mmol})$. The reaction mixture was degassed with $\mathrm{Ar}_{2}$ and stirred at $120{ }^{\circ} \mathrm{C}$ for $18 \mathrm{~h}$. After cooling to $\mathrm{rt}$, the reaction was quenched by addition of saturated aqueous $\mathrm{NaCl}(40 \mathrm{~mL})$ and was extracted with EtOAc $(3 \times 20$ $\mathrm{mL})$. The combined organic phase was washed with brine $(20 \mathrm{~mL} \times 5)$ and dried over anhydrous $\mathrm{Na}_{2} \mathrm{SO}_{4}$. The residue was purified by column chromatography on silica gel $\left(2: 1,60-90{ }^{\circ} \mathrm{C}\right.$ petroleum ether - EtOAc) to give the desired intermediate $65 \mathbf{a}$. Off-white solid (191 mg, 27.2 \%). ${ }^{1} \mathrm{H}$ NMR (400 MHz, Chloroform- $d$ ) $\delta 7.48(\mathrm{~d}, J=$ 
$8.3 \mathrm{~Hz}, 2 \mathrm{H}), 6.81(\mathrm{~d}, J=1.5 \mathrm{~Hz}, 1 \mathrm{H}), 6.74(\mathrm{dd}, J=8.0,1.5 \mathrm{~Hz}, 1 \mathrm{H}), 4.60(\mathrm{~s}, 2 \mathrm{H})$, $3.60(\mathrm{~s}, 3 \mathrm{H})$.

The intermediate 66a was obtained from 65a and 58e through a procedure similar to the preparation of the intermediate 62 . Light brown solid. $8.2 \%$ yield. ${ }^{1} \mathrm{H}$ NMR (400 MHz, Chloroform- $d$ ) $\delta 7.68(\mathrm{~s}, 1 \mathrm{H}), 7.29(\mathrm{~d}, J=8.1 \mathrm{~Hz}, 1 \mathrm{H}), 6.89(\mathrm{~s}, 2 \mathrm{H}), 6.46$ $(\mathrm{d}, J=8.1 \mathrm{~Hz}, 1 \mathrm{H}), 6.38(\mathrm{~s}, 1 \mathrm{H}), 4.41(\mathrm{~s}, 2 \mathrm{H}), 4.22(\mathrm{~d}, J=5.9 \mathrm{~Hz}, 2 \mathrm{H}), 3.70(\mathrm{~s}, 3 \mathrm{H})$, $1.97(\mathrm{~s}, 6 \mathrm{H}), 1.46(\mathrm{~s}, 9 \mathrm{H})$.

The compound 66a $(20 \mathrm{mg}, 46.3 \mu \mathrm{mol})$ was dissolved in $4 \mathrm{M} \mathrm{HCl}$ in 1, 4-dioxane solution $(4 \mathrm{~mL})$ at room temperature and stirred for $1 \mathrm{~h}$. The solvent was removed in vacuoto obtain the desired product $\mathbf{1 5}$ as a hydrochloride salt. Yellow amorphous solid (16 mg, 96\%). ${ }^{1} \mathrm{H}$ NMR (400 MHz, Methanol- $\left.d_{4}\right) \delta 9.11(\mathrm{~s}, 1 \mathrm{H}), 7.37$ (d, J = 8.0 Hz, 1H), $7.25(\mathrm{~s}, 2 \mathrm{H}), 6.76(\mathrm{~d}, \mathrm{~J}=1.5 \mathrm{~Hz}, 1 \mathrm{H}), 6.54(\mathrm{dd}, \mathrm{J}=8.0,1.5 \mathrm{~Hz}, 1 \mathrm{H}), 4.08(\mathrm{~s}, 2 \mathrm{H})$, $3.91(\mathrm{~s}, 3 \mathrm{H}), 2.16(\mathrm{~s}, 6 \mathrm{H}) .{ }^{13} \mathrm{C} \mathrm{NMR}\left(126 \mathrm{MHz}\right.$, Methanol- $\left.d_{4}\right) \delta$ 152.99, 141.24, $137.93,136.75,134.42,132.74,132.40,130.88,130.79,129.32,127.26,122.83$, $118.08,117.80,117.16,97.06,43.82,35.36,20.38(* 2 \mathrm{C}) . \mathrm{HRMS}(\mathrm{ESI}): m / z[\mathrm{M}+\mathrm{H}]^{+}$ calcd for $\mathrm{C}_{20} \mathrm{H}_{22} \mathrm{~N}_{5} 322.1870$; found 322.1866. HPLC: $t_{\mathrm{R}}=1.922 \mathrm{~min}, 100 \%$.

4-(4-(4-(aminomethyl)-2,6-dimethylphenyl)-1-methyl-1H-imidazol-5-yl)-2-(methyla mino)benzonitrile (16). To a mixture of 2-amino-4-bromobenzonitrile (64a, $300 \mathrm{mg}$, $1.52 \mathrm{mmol})$ and $\mathrm{K}_{2} \mathrm{CO}_{3}(421 \mathrm{mg}, 3.05 \mathrm{mmol})$ in $N, N$-dimethylformamide (10 mL) was added $\mathrm{CH}_{3} \mathrm{I}(105 \mu \mathrm{L}, 1.67 \mathrm{mmol})$ at room temperature. The resulting mixture was stirred overnight before quenched by $2 \mathrm{M} \mathrm{NaOH}$ aqueous solution and was extracted with EtOAc $(3 \times 20 \mathrm{~mL})$. The combined organic phase was washed with brine $(20$ 
$\mathrm{mL} \times 5)$ and dried over anhydrous $\mathrm{Na}_{2} \mathrm{SO}_{4}$, filtered, and concentrated under reduced pressure. The residue was purified by column chromatography on silica gel $(3: 1,60$ $90{ }^{\circ} \mathrm{C}$ petroleum ether - EtOAc) to give the desired intermediate $\mathbf{6 4 b}$. White solid. (148 mg, $46.0 \%)$.

The intermediate $\mathbf{6 5 b}$ was obtained from 63 and $64 \mathrm{~b}$ through a procedure similar to the preparation of the intermediate $\mathbf{6 5 a}$. White solid. $34.1 \%$ yield. ${ }^{1} \mathrm{H}$ NMR (400 MHz, Chloroform- $d) \delta 7.44(\mathrm{~d}, J=7.9 \mathrm{~Hz}, 2 \mathrm{H}), 6.68(\mathrm{~d}, J=1.4 \mathrm{~Hz}, 1 \mathrm{H}), 6.65(\mathrm{dd}, J=7.9$, $1.4 \mathrm{~Hz}, 1 \mathrm{H}), 4.86(\mathrm{~s}, 1 \mathrm{H}), 3.60(\mathrm{~s}, 3 \mathrm{H}), 2.94(\mathrm{~s}, 3 \mathrm{H})$.

The intermediate $66 \mathrm{~b}$ was obtained from $65 \mathrm{~b}$ and $58 \mathrm{e}$ through a procedure similar to the preparation of the intermediate 62. White solid. $7.8 \%$ yield. ${ }^{1} \mathrm{H}$ NMR $(400 \mathrm{MHz}$, Chloroform- $d$ ) $\delta 7.69(\mathrm{~s}, 1 \mathrm{H}), 7.32(\mathrm{~d}, J=8.0 \mathrm{~Hz}, 1 \mathrm{H}), 6.91(\mathrm{~s}, 2 \mathrm{H}), 6.49(\mathrm{dd}, J=8.0$, $1.5 \mathrm{~Hz}, 1 \mathrm{H}), 6.21(\mathrm{~s}, 1 \mathrm{H}), 4.54(\mathrm{~d}, J=5.2 \mathrm{~Hz}, 1 \mathrm{H}), 4.22(\mathrm{~d}, J=5.8 \mathrm{~Hz}, 2 \mathrm{H}), 3.76(\mathrm{~s}$, 3H), $2.57(\mathrm{~d}, J=4.9 \mathrm{~Hz}, 3 \mathrm{H}), 1.99(\mathrm{~s}, 6 \mathrm{H}), 1.46(\mathrm{~s}, 9 \mathrm{H})$.

The target compound $\mathbf{1 6}$ was prepared from $\mathbf{6 6 \mathbf { b }}$ by following a similar method as described for compound 15. Light yellow amorphous solid (6 mg, 86.5\%). ${ }^{1} \mathrm{H}$ NMR (400 MHz, Methanol- $\left.d_{4}\right) \delta 9.06(\mathrm{~s}, 1 \mathrm{H}), 7.43(\mathrm{~d}, \mathrm{~J}=7.9 \mathrm{~Hz}, 1 \mathrm{H}), 7.26(\mathrm{~s}, 2 \mathrm{H}), 6.64-$ $6.59(\mathrm{~m}, 1 \mathrm{H}), 6.55(\mathrm{~s}, 1 \mathrm{H}), 4.09(\mathrm{~s}, 2 \mathrm{H}), 3.94(\mathrm{~s}, 3 \mathrm{H}), 2.70(\mathrm{~s}, 3 \mathrm{H}), 2.16(\mathrm{~s}, 6 \mathrm{H}) .{ }^{13} \mathrm{C}$ NMR (151 MHz, Methanol- $\left.d_{4}\right) \delta 152.83,141.26(* 2 \mathrm{C}), 138.11,136.65,134.88$, 133.06, 133.01, 132.88, 130.88, 129.28(*2C), 117.86, 117.34, 112.38, 97.44, 43.81, 35.38, 29.91, 20.36(*2C). HRMS (ESI): $m / z[\mathrm{M}+\mathrm{H}]^{+}$calcd for $\mathrm{C}_{21} \mathrm{H}_{24} \mathrm{~N}_{5}$ 346.2026; found 346.2036. HPLC: $t_{\mathrm{R}}=2.073 \mathrm{~min}, 100 \%$. 
nzonitrile (17). (4-cyano-3-fluorophenyl)boronic acid (1.48 g, $9.0 \mathrm{mmol})$ and 5-bromo-1-methyl-1H-imidazole $(\mathbf{6 7}, 966 \mathrm{mg}, 6.0 \mathrm{mmol})$ was suspended in EtOH/ $\mathrm{H}_{2} \mathrm{O} /$ toluene $(30 \mathrm{~mL} / 3 \mathrm{~mL} / 6 \mathrm{~mL})$, followed by addition of $\mathrm{Na}_{2} \mathrm{CO}_{3}(2.83 \mathrm{~g}$, $27.0 \mathrm{mmol})$ and $\mathrm{Pd}(\mathrm{dppf}) \mathrm{Cl}_{2}(200 \mathrm{mg}, 0.3 \mathrm{mmol})$ under argon atmosphere. The mixture was stirred at $100{ }^{\circ} \mathrm{C}$ for $10 \mathrm{~h}$ and was then concentrated and purified by column chromatography on silica gel $\left(4: 1,60-90{ }^{\circ} \mathrm{C}\right.$ petroleum ether - EtOAc $)$ to afford the intermediate 68a. Yellow solid (900 mg, 75\% yield). ${ }^{1} \mathrm{H}$ NMR (500 MHz, Chloroform- $d$ ) $\delta 7.69(\mathrm{dd}, J=6.5,8.0 \mathrm{~Hz}, 1 \mathrm{H}), 7.59(\mathrm{~s}, 1 \mathrm{H}), 7.32(\mathrm{dd}, J=1.5,7.0 \mathrm{~Hz}$, 1H), 7.28-7.26 (m, 2H), $3.67(\mathrm{~s}, 3 \mathrm{H})$.

To a stirred solution of intermediate $\mathbf{6 8 a}(201 \mathrm{mg}, 1.0 \mathrm{mmol})$ in $\mathrm{MeCN}(20 \mathrm{ml})$ was added NBS (201 mg, $1.0 \mathrm{mmol}, 1.0$ equiv) at $-20^{\circ} \mathrm{C}$. The mixture was slowly warmed to room temperature and stirred overnight. The reaction was slowly quenched by saturated aqueous $\mathrm{Na}_{2} \mathrm{~S}_{2} \mathrm{O}_{3}$ and was extracted with EtOAc $(3 \times 10 \mathrm{~mL})$. The combined organic phase was washed with brine $(10 \mathrm{~mL})$ and dried over anhydrous $\mathrm{Na}_{2} \mathrm{SO}_{4}$, filtered, and concentrated in vacuo. The residue was purified by column chromatography on silica gel $\left(5: 1,60-90^{\circ} \mathrm{C}\right.$ petroleum ether - EtOAc) to give the intermediate 69a. White solid (150 mg, 54\% yield). ${ }^{1} \mathrm{H}$ NMR (500 MHz, Chloroform- $d$ ) $\delta$ 7.76-7.73 (m, 1H), $7.52(\mathrm{~s}, 1 \mathrm{H})$, 7.36-7.31 (m, 2H), $3.66(\mathrm{~s}, 3 \mathrm{H})$.

The target compound 17 was prepared from 69a and 58e by following a similar method as described for compound 7. White amorphous solid (5 mg, 7.5\% yield over two steps). ${ }^{1} \mathrm{H}$ NMR (600 MHz, Methanol- $\left.d_{4}\right) \delta 9.30(\mathrm{~s}, 1 \mathrm{H}), 7.86(\mathrm{~s}, 1 \mathrm{H}), 7.42-7.35$ (m, 2H), $7.29(\mathrm{~s}, 2 \mathrm{H}), 4.10(\mathrm{~s}, 2 \mathrm{H}), 3.99(\mathrm{~s}, 3 \mathrm{H}), 2.16(\mathrm{~s}, 6 \mathrm{H}) .{ }^{13} \mathrm{C}$ NMR (151 MHz, 
Methanol- $\left.d_{4}\right) \delta 164.93,163.21,141.21(* 2 \mathrm{C}), 138.82,137.21,135.92,134.09(\mathrm{~d}, \mathrm{~J}=$ $8.4 \mathrm{~Hz}), 130.85(\mathrm{~d}, \mathrm{~J}=42.6 \mathrm{~Hz}), 129.60(* 2 \mathrm{C}), 128.03,127.08,119.01(\mathrm{~d}, \mathrm{~J}=21.5$ Hz), 113.81, $103.94(\mathrm{~d}, \mathrm{~J}=15.2 \mathrm{~Hz}), 43.84,36.22,20.57(* 2 \mathrm{C}) . \mathrm{HRMS}(\mathrm{ESI}): \mathrm{m} / z$ [M $+\mathrm{H}]^{+}$calcd for $\mathrm{C}_{20} \mathrm{H}_{20} \mathrm{FN}_{4}$ 335.1667; found 335.1664. HPLC: $t_{\mathrm{R}}=2.054$ min, $100 \%$. 4-(4-(4-(aminomethyl)-2,6-dimethylphenyl)-1-methyl-1H-imidazol-5-yl)-2-chlorobe nzonitrile (18). The intermediate $\mathbf{6 8 b}$ was obtained from 67 through a procedure similar to the preparation of the intermediate 68a. Yellow solid (500 mg, 90\% yield). ${ }^{1} \mathrm{H}$ NMR (500 MHz, Chloroform- $d$ ) $\delta 7.73$ (d, J=8.5 Hz, 1H), 7.60 (brs, 1H), 7.55 (d, $J=2.0 \mathrm{~Hz}, 1 \mathrm{H}), 7.32(\mathrm{dd}, J=1.5,9.5 \mathrm{~Hz}, 1 \mathrm{H}), 7.28(\mathrm{brs}, 1 \mathrm{H}), 3.75(\mathrm{~s}, 3 \mathrm{H})$.

The intermediate $69 \mathrm{~b}$ was obtained from $\mathbf{6 8 b}$ through a procedure similar to the preparation of the intermediate 69a. Yellow solid (436 mg, 64\% yield). ${ }^{1} \mathrm{H}$ NMR (500 $\left.\mathrm{MHz}, \mathrm{MeOD}-d_{4}\right) \delta 7.97(\mathrm{~d}, J=8.5 \mathrm{~Hz}, 1 \mathrm{H}), 7.84-7.81(\mathrm{~m}, 2 \mathrm{H}), 7.65(\mathrm{dd}, J=1.5,9.5$ $\mathrm{Hz}, 1 \mathrm{H}), 3.72$ (s, 3H).

The target compound 18 was prepared from $69 \mathrm{~b}$ and $\mathbf{5 8 e}$ by following a similar method as described for compound 7. White amorphous solid (6 mg, 8.6\% yield over two steps). ${ }^{1} \mathrm{H}$ NMR (500 MHz, MeOD- $\left.d_{4}\right): \delta 8.84(\mathrm{~s}, 1 \mathrm{H}), 7.85(\mathrm{~d}, J=8.0 \mathrm{~Hz}, 1 \mathrm{H})$, $7.59(\mathrm{~s}, 1 \mathrm{H}), 7.43(\mathrm{~d}, J=8.0 \mathrm{~Hz}, 1 \mathrm{H}), 7.25(\mathrm{~s}, 2 \mathrm{H}), 4.10(\mathrm{~s}, 2 \mathrm{H}), 3.92(\mathrm{~s}, 3 \mathrm{H}), 1.31(\mathrm{~s}$, 6H). HRMS (ESI): $m / z[\mathrm{M}+\mathrm{H}]^{+}$calcd for $\mathrm{C}_{20} \mathrm{H}_{20} \mathrm{ClN}_{4}$ 351.1371; found 351.1372. HPLC: $t_{\mathrm{R}}=2.083 \mathrm{~min}, 100 \%$.

4-(4-(4-(aminomethyl)-2,6-dimethylphenyl)-1-methyl-1H-imidazol-5-yl)-2-methoxy benzonitrile (19). The intermediate $68 \mathrm{c}$ was obtained from 67 through a procedure similar to the preparation of the intermediate 68a. Yellow amorphous solid (640 mg, 
$56 \%$ yield). ${ }^{1} \mathrm{H}$ NMR (500 MHz, Chloroform- $d$ ) $\delta 7.62(\mathrm{~d}, J=8.5 \mathrm{~Hz}, 1 \mathrm{H}), 7.57$ (brs, 1H), 7.22 (brs, 1H), $7.02(\mathrm{dd}, J=1.5,8.5 \mathrm{~Hz}, 1 \mathrm{H}), 6.97$ (d, $J=1.5 \mathrm{~Hz}, 1 \mathrm{H}), 3.97(\mathrm{~s}$, 3H), $3.72(\mathrm{~s}, 3 \mathrm{H})$.

The intermediate 69c was obtained from 68c through a procedure similar to the preparation of the intermediate 69a. Yellow solid (240 mg, 82\% yield). ${ }^{1} \mathrm{H}$ NMR (500 MHz, Chloroform- $d) \delta 7.66(\mathrm{~d}, J=8.0 \mathrm{~Hz}, 1 \mathrm{H}), 7.50(\mathrm{~s}, 1 \mathrm{H}), 7.08(\mathrm{~d}, J=1.5 \mathrm{~Hz}, 1 \mathrm{H})$, $7.03(\mathrm{dd}, J=1.5,8.0 \mathrm{~Hz}, 1 \mathrm{H}), 3.96(\mathrm{~s}, 3 \mathrm{H}), 3.64(\mathrm{~s}, 3 \mathrm{H})$.

The target compound 19 was prepared from 69c and 58e by following a similar method as described for compound 7. White amorphous solid (8 mg, 11.6\% yield over two steps). ${ }^{1} \mathrm{H}$ NMR (600 MHz, Methanol- $\left.d_{4}\right) \delta 9.26(\mathrm{~s}, 1 \mathrm{H}), 7.67(\mathrm{~d}, \mathrm{~J}=7.9 \mathrm{~Hz}, 1 \mathrm{H})$, $7.29(\mathrm{~s}, 2 \mathrm{H}), 7.13(\mathrm{~s}, 1 \mathrm{H}), 7.05(\mathrm{~d}, \mathrm{~J}=7.8 \mathrm{~Hz}, 1 \mathrm{H}), 4.10(\mathrm{~s}, 2 \mathrm{H}), 4.00(\mathrm{~s}, 3 \mathrm{H}), 3.84(\mathrm{~s}$, 3H), $2.17(\mathrm{~s}, 6 \mathrm{H}) .{ }^{13} \mathrm{C}$ NMR (151 MHz, Methanol- $\left.d_{4}\right) \delta 162.65,141.29,138.16$, 137.10, 135.59(*2C), 133.14, 132.23, 130.17, 129.47(*2C), 127.54, 123.18, 116.18, 114.20, 104.16, 57.23, 43.75, 35.79, 20.38(*2C). HRMS (ESI): $m / z[M+H]^{+}$calcd for $\mathrm{C}_{21} \mathrm{H}_{23} \mathrm{~N}_{4} \mathrm{O}$ 347.1866; found 347.1863. HPLC: $t_{\mathrm{R}}=2.083 \mathrm{~min}, 100 \%$.

4-(4-(4-(aminomethyl)-2,6-dimethylphenyl)-1-methyl-1H-imidazol-5-yl)-2-(trifluoro methoxy)benzonitrile (20). The intermediate $\mathbf{6 8 d}$ was obtained from $\mathbf{6 7}$ through a procedure similar to the preparation of the intermediate 68a. Yellow solid ( $850 \mathrm{mg}, 44 \%$ yield). ${ }^{1} \mathrm{H}$ NMR (500 MHz, Chloroform- $\left.d\right) \delta 7.91(\mathrm{~d}, J=8.0 \mathrm{~Hz}, 1 \mathrm{H}), 7.82(\mathrm{~s}, 1 \mathrm{H})$, $7.71(\mathrm{dd}, J=1.5,8.0 \mathrm{~Hz}, 1 \mathrm{H}), 7.67-7.61(\mathrm{~m}, 2 \mathrm{H}), 3.77(\mathrm{~s}, 3 \mathrm{H})$.

The intermediate 69d was obtained from 68d through a procedure similar to the preparation of the intermediate 69a. Yellow solid (670 mg, 57\% yield). ${ }^{1} \mathrm{H}$ NMR (500 
MHz, Chloroform- $d$ ) $\delta 7.97(\mathrm{~d}, J=8.0 \mathrm{~Hz}, 1 \mathrm{H}), 7.87(\mathrm{~s}, 1 \mathrm{H}), 7.78(\mathrm{dd}, J=1.5,8.0 \mathrm{~Hz}$, $1 \mathrm{H}), 7.55(\mathrm{~s}, 1 \mathrm{H}), 3.67(\mathrm{~s}, 3 \mathrm{H})$.

The target compound 20 was prepared from 69d and 58e by following a similar method as described for compound 7. White amorphous solid (12 mg, 15.6\% yield over two steps). ${ }^{1} \mathrm{H}$ NMR (500 MHz, MeOD- $\left.d_{4}\right): \delta 9.06$ (s, $\left.1 \mathrm{H}\right), 8.08(\mathrm{~d}, J=8.0 \mathrm{~Hz}$, 1H), $7.88(\mathrm{~s}, 1 \mathrm{H}), 7.81(\mathrm{dd}, J=1.5,8.0 \mathrm{~Hz}, 1 \mathrm{H}), 7.26(\mathrm{~s}, 2 \mathrm{H}), 4.10(\mathrm{~s}, 2 \mathrm{H}), 3.96(\mathrm{~s}$, 3H), $2.145(\mathrm{~s}, 6 \mathrm{H})$. HRMS (ESI): $m / z[\mathrm{M}+\mathrm{H}]^{+}$calcd for $\mathrm{C}_{21} \mathrm{H}_{20} \mathrm{~F}_{3} \mathrm{~N}_{4}$ 385.1605; found 385.0275. HPLC: $t_{\mathrm{R}}=4.211 \mathrm{~min}, 95.31 \%$.

\section{4-(4-(4-cyano-2,6-dimethylphenyl)-1-methyl-1H-imidazol-5-yl)-1-naphthonitrile}

(21). Compound 61a (100 mg, $321.36 \mu \mathrm{mol}), 71 \mathrm{a}$ (99 mg, $385.63 \mu \mathrm{mol})$, tricyclohexylphosphane $(9 \mathrm{mg}, 32.14 \mu \mathrm{mol})$ and $\mathrm{K}_{3} \mathrm{PO}_{4}(137 \mathrm{mg}, 642.71 \mu \mathrm{mol})$ were suspended in toluene $(5 \mathrm{~mL})$. The mixture was degassed with $\operatorname{Ar}_{2}$ and $\operatorname{Pd}_{2}(\mathrm{dba})_{3}(30$ mg, $32.14 \mu \mathrm{mol})$ was added quickly under argon atmosphere. The reaction mixture was heated to $110{ }^{\circ} \mathrm{C}$ and stirred overnight. After completion of the reaction, the mixture was concentrated under reduced pressure and purified by column chromatography on silica gel $\left(2: 1,60-90{ }^{\circ} \mathrm{C}\right.$ petroleum ether - EtOAc) to give the desired compound 21. Yellow amorphous solid (15 mg, 12.9\%). ${ }^{1} \mathrm{H}$ NMR (500 MHz, Chloroform- $d$ ) $\delta 8.31(\mathrm{~d}, \mathrm{~J}=8.4 \mathrm{~Hz}, 1 \mathrm{H}), 7.85(\mathrm{~s}, 1 \mathrm{H}), 7.82(\mathrm{~d}, \mathrm{~J}=7.4 \mathrm{~Hz}, 1 \mathrm{H}), 7.73$ $(\mathrm{ddd}, \mathrm{J}=8.2,6.8,1.2 \mathrm{~Hz}, 1 \mathrm{H}), 7.69(\mathrm{~d}, \mathrm{~J}=8.1 \mathrm{~Hz}, 1 \mathrm{H}), 7.63(\mathrm{ddd}, \mathrm{J}=8.3,6.7,1.1 \mathrm{~Hz}$ 1H), $7.30(\mathrm{~s}, 1 \mathrm{H}), 7.21(\mathrm{~d}, \mathrm{~J}=7.4 \mathrm{~Hz}, 1 \mathrm{H}), 7.11(\mathrm{~s}, 1 \mathrm{H}), 3.46(\mathrm{~s}, 3 \mathrm{H}), 2.26(\mathrm{~s}, 3 \mathrm{H})$, $1.86(\mathrm{~s}, 3 \mathrm{H}) .{ }^{13} \mathrm{C}$ NMR $(126 \mathrm{MHz}$, Chloroform- $d) \delta 139.54,138.81,138.78,138.05$, $137.86,132.27,131.90,131.45,131.09,130.21,130.19,128.49,127.99,127.61$, 
$127.05,125.73,125.32,118.47,116.72,111.09,110.94,32.37,20.27,20.02$. HRMS (ESI): $m / z[\mathrm{M}+\mathrm{H}]^{+}$calcd for $\mathrm{C}_{24} \mathrm{H}_{19} \mathrm{~N}_{4}$ 363.1604; found 363.1603. HPLC: $t_{\mathrm{R}}=2.912$ $\min , 96.04 \%$.

\section{4-(4-(4-formyl-2,6-dimethylphenyl)-1-methyl-1H-imidazol-5-yl)-1-naphthonitrile}

(22). The target compound 22 was prepared from $61 \mathrm{a}$ and $55 \mathrm{c}$ by following a similar method as described for compound 62. Yellow amorphous solid (21 mg, 18.3\% yield). Purity > 90\%. ${ }^{1} \mathrm{H}$ NMR (400 MHz, Chloroform- $d$ ) $\delta 9.85(\mathrm{~s}, 1 \mathrm{H}), 8.36-8.26(\mathrm{~m}, 1 \mathrm{H})$, $7.84(\mathrm{~s}, 1 \mathrm{H}), 7.80(\mathrm{~d}, \mathrm{~J}=7.4 \mathrm{~Hz}, 1 \mathrm{H}), 7.76-7.70(\mathrm{~m}, 2 \mathrm{H}), 7.66-7.60(\mathrm{~m}, 1 \mathrm{H}), 7.52$ $(\mathrm{d}, \mathrm{J}=1.5 \mathrm{~Hz}, 1 \mathrm{H}), 7.33(\mathrm{~s}, 1 \mathrm{H}), 7.22(\mathrm{~d}, \mathrm{~J}=7.4 \mathrm{~Hz}, 1 \mathrm{H}), 3.47(\mathrm{~s}, 4 \mathrm{H}), 2.32(\mathrm{~s}, 3 \mathrm{H})$, 1.90 (s, 3H). ${ }^{13} \mathrm{C}$ NMR (151 MHz, Chloroform- $\left.d\right) \delta 192.65,139.91,139.86,139.31$, $139.11,139.03,135.68,132.87,132.64,132.09,131.73,129.06,128.79,128.57(* 2 C)$, 128.29, 127.57, 126.30, 126.04, 117.40, 111.57, 33.04, 21.02, 20.76. HRMS (ESI): $m / z[\mathrm{M}+\mathrm{H}]^{+}$calcd for $\mathrm{C}_{24} \mathrm{H}_{20} \mathrm{~N}_{3} \mathrm{O} 366.1601$; found 366.1594. HPLC: $t_{\mathrm{R}}=2.892 \mathrm{~min}$, $95.40 \%$.

4-(4-(2,6-dimethyl-4-propionylphenyl)-1-methyl-1H-imidazol-5-yl)-1-naphthonitril $e$ (23). The target compound 23 was prepared from $61 \mathrm{a}$ and $71 \mathrm{~b}$ by following a similar method as described for compound 21. Yellow amorphous solid (41 mg, 32.8\% yield). ${ }^{1} \mathrm{H}$ NMR (400 MHz, Chloroform- $d$ ) $\delta 8.33-8.25$ (m, 1H), 7.91 (s, 1H), 7.79 $(\mathrm{d}, J=7.4 \mathrm{~Hz}, 1 \mathrm{H}), 7.75-7.68(\mathrm{~m}, 2 \mathrm{H}), 7.67-7.60(\mathrm{~m}, 1 \mathrm{H}), 7.59(\mathrm{~d}, J=1.8 \mathrm{~Hz}, 1 \mathrm{H})$, $7.40(\mathrm{~d}, J=1.8 \mathrm{~Hz}, 1 \mathrm{H}), 7.22(\mathrm{~d}, J=7.4 \mathrm{~Hz}, 1 \mathrm{H}), 3.47(\mathrm{~s}, 3 \mathrm{H}), 2.89(\mathrm{q}, J=7.2 \mathrm{~Hz}$ 2H), $2.29(\mathrm{~s}, 3 \mathrm{H}), 1.87(\mathrm{~s}, 3 \mathrm{H}), 1.15(\mathrm{t}, J=7.2 \mathrm{~Hz}, 3 \mathrm{H})$. LRMS (ESI): $m / z[\mathrm{M}+\mathrm{H}]^{+}$ calcd for $\mathrm{C}_{26} \mathrm{H}_{24} \mathrm{~N}_{3} \mathrm{O} 394.19$; found 394.33. 
onitrile (24). To a solution of compound $22(20 \mathrm{mg}, 54.73 \mu \mathrm{mol})$ in anhydrous $\mathrm{CH}_{3} \mathrm{OH}(3 \mathrm{~mL})$ was added $\mathrm{NaBH}_{4}(4 \mathrm{mg}, 109.46 \mu \mathrm{mol})$, and the resulting mixture was stirred at room temperature for $5 \mathrm{~h}$. Then, the reaction was quenched by saturated aqueous $\mathrm{NaCl}$, and the resulting mixture was extracted with $\mathrm{DCM}-\mathrm{CH}_{3} \mathrm{OH}(10: 1,3$ $\times 50 \mathrm{~mL}$ ). The combined organic phase was dried over anhydrous $\mathrm{Na}_{2} \mathrm{SO}_{4}$, filtered, and concentrated under reduced pressure. The residue was purified by column chromatography on silica gel (30:1, DCM - MeOH) to give the compound 24. Off-white amorphous solid (15 mg, $74.6 \%$ ). ${ }^{1} \mathrm{H}$ NMR (400 MHz, Methanol- $\left.d_{4}\right) \delta$ $9.35(\mathrm{~s}, 1 \mathrm{H}), 8.30(\mathrm{~d}, \mathrm{~J}=8.1 \mathrm{~Hz}, 1 \mathrm{H}), 8.03(\mathrm{~d}, \mathrm{~J}=7.4 \mathrm{~Hz}, 1 \mathrm{H}), 7.93(\mathrm{~d}, \mathrm{~J}=8.2 \mathrm{~Hz}$, 1H), $7.86(\mathrm{t}, \mathrm{J}=7.2 \mathrm{~Hz}, 1 \mathrm{H}), 7.82-7.77(\mathrm{~m}, 1 \mathrm{H}), 7.61(\mathrm{~d}, \mathrm{~J}=7.4 \mathrm{~Hz}, 1 \mathrm{H}), 7.12(\mathrm{~s}$, 1H), $6.95(\mathrm{~s}, 1 \mathrm{H}), 4.47(\mathrm{~s}, 2 \mathrm{H}), 3.71(\mathrm{~s}, 3 \mathrm{H}), 2.30(\mathrm{~s}, 3 \mathrm{H}), 1.97$ (s, 3H). ${ }^{13} \mathrm{C}$ NMR (151 MHz, Methanol- $\left.d_{4}\right) \delta 145.41,140.42,139.89,138.16,133.73,133.28,132.81,132.05$, $130.95,130.86,130.78,130.53,129.40,127.31,127.24,126.97,126.82,125.10$ 117.67, 114.22, 64.39, 35.40, 20.70, 20.64. HRMS (ESI): $m / z[\mathrm{M}+\mathrm{H}]^{+}$calcd for $\mathrm{C}_{24} \mathrm{H}_{22} \mathrm{~N}_{3} \mathrm{O}$ 368.1757; found 368.1758. HPLC: $t_{\mathrm{R}}=2.647 \mathrm{~min}, 100 \%$.

4-(4-(4-(1-hydroxypropyl)-2,6-dimethylphenyl)-1-methyl-1H-imidazol-5-yl)-1-naph thonitrile (25). The target compound 25 was prepared from 23 by following a similar method as described for compound 24. Light yellow amorphous solid (13 mg, 66.7\% yield). ${ }^{1} \mathrm{H}$ NMR (600 MHz, Methanol- $\left.d_{4}\right) \delta 8.23(\mathrm{~d}, \mathrm{~J}=8.3 \mathrm{~Hz}, 1 \mathrm{H}), 8.04(\mathrm{~s}, 1 \mathrm{H})$, $7.92(\mathrm{~d}, \mathrm{~J}=7.4 \mathrm{~Hz}, 1 \mathrm{H}), 7.80(\mathrm{~d}, \mathrm{~J}=8.4 \mathrm{~Hz}, 1 \mathrm{H}), 7.79-7.75(\mathrm{~m}, 1 \mathrm{H}), 7.71-7.67(\mathrm{~m}$, 1H), $7.41(\mathrm{dd}, \mathrm{J}=7.4,4.5 \mathrm{~Hz}, 1 \mathrm{H}), 6.97(\mathrm{~d}, \mathrm{~J}=29.5 \mathrm{~Hz}, 1 \mathrm{H}), 6.82(\mathrm{~d}, \mathrm{~J}=30.5 \mathrm{~Hz}$, 
1H), $4.34(\mathrm{q}, \mathrm{J}=6.9 \mathrm{~Hz}, 1 \mathrm{H}), 3.51(\mathrm{~s}, 3 \mathrm{H}), 2.21(\mathrm{~d}, \mathrm{~J}=2.6 \mathrm{~Hz}, 3 \mathrm{H}), 1.88(\mathrm{~d}, \mathrm{~J}=4.7$ $\mathrm{Hz}, 3 \mathrm{H}), 1.64$ (ddq, $\mathrm{J}=33.8,12.7,7.1 \mathrm{~Hz}, 2 \mathrm{H}), 0.79(\mathrm{t}, \mathrm{J}=7.4 \mathrm{~Hz}, 3 \mathrm{H}) .{ }^{13} \mathrm{C} \mathrm{NMR}$ (151 MHz, Methanol-d4) $\delta$ 144.56, 138.93, 138.89, 138.27 (d, J = 19.9 Hz), 137.58 (d, $\mathrm{J}=27.3 \mathrm{~Hz}), 132.87,132.41,131.87,131.82,131.67,128.74,128.38,128.09(\mathrm{~d}, \mathrm{~J}=$ 4.1 Hz), 127.36, 126.24, 125.13, 125.00, $124.36(\mathrm{~d}, \mathrm{~J}=9.2 \mathrm{~Hz}), 116.77,110.62,74.97$, 31.75, 31.28, 19.67, 19.50, 9.06. HRMS (ESI): $m / z[M+H]^{+}$calcd for $\mathrm{C}_{26} \mathrm{H}_{26} \mathrm{~N}_{3} \mathrm{O}$ 396.2070; found 396.2071. HPLC: $t_{\mathrm{R}}=2.839 \mathrm{~min}, 98.09 \%$.

tert-butyl

(4-(5-(4-cyanonaphthalen-1-yl)-1-methyl-1H-imidazol-4-yl)-3,5-dimethylphenyl)carb amate (26). The target compound 26 was prepared from 61a and $71 \mathrm{~b}$ by following a similar method as described for compound 62. White amorphous solid ( $25 \mathrm{mg}, 9.2 \%$ yield). ${ }^{1} \mathrm{H}$ NMR (600 MHz, Chloroform- $\left.d\right) \delta 8.27(\mathrm{~d}, \mathrm{~J}=8.3 \mathrm{~Hz}, 1 \mathrm{H}), 7.79(\mathrm{~d}, \mathrm{~J}=7.5$ $\mathrm{Hz}, 2 \mathrm{H}), 7.73-7.66(\mathrm{~m}, 2 \mathrm{H}), 7.62-7.57(\mathrm{~m}, 1 \mathrm{H}), 7.23(\mathrm{~d}, \mathrm{~J}=7.4 \mathrm{~Hz}, 1 \mathrm{H}), 7.03(\mathrm{~s}$, 1H), $6.78(\mathrm{~s}, 1 \mathrm{H}), 6.51-6.45(\mathrm{~m}, 1 \mathrm{H}), 3.42(\mathrm{~s}, 3 \mathrm{H}), 2.19(\mathrm{~s}, 3 \mathrm{H}), 1.77(\mathrm{~s}, 3 \mathrm{H}), 1.46(\mathrm{~s}$, 9H). ${ }^{13} \mathrm{C}$ NMR (151 MHz, Chloroform-d) $\delta 152.84,140.00,139.47,138.80,138.76$, $137.65,133.38,132.79,132.17,131.91,128.80,128.37,128.25,128.20,127.46$, $126.27,126.07,117.59,117.04,117.01,110.97,80.35,32.87,28.42(* 3 \mathrm{C}), 21.10$, 20.83. HRMS (ESI): $m / z[\mathrm{M}+\mathrm{H}]^{+}$calcd for $\mathrm{C}_{28} \mathrm{H}_{29} \mathrm{~N}_{4} \mathrm{O}_{2}$ 453.2285; found 453.2288. HPLC: $t_{\mathrm{R}}=3.310 \mathrm{~min}, 95.75 \%$.

4-(4-(4-amino-2,6-dimethylphenyl)-1-methyl-1H-imidazol-5-yl)-1-naphthonitrile (27). The target compound $\mathbf{2 7}$ was prepared from $\mathbf{2 6}$ by following a similar method as described for compound 13. White amorphous solid (10 mg, 85\% yield). ${ }^{1} \mathrm{H}$ NMR 
$\left(500 \mathrm{MHz}\right.$, Methanol- $\left.d_{4}\right) \delta 9.43(\mathrm{~s}, 1 \mathrm{H}), 8.30(\mathrm{~d}, \mathrm{~J}=8.1 \mathrm{~Hz}, 1 \mathrm{H}), 8.06(\mathrm{~d}, \mathrm{~J}=7.4 \mathrm{~Hz}$, 1H), $7.95(\mathrm{~d}, \mathrm{~J}=8.3 \mathrm{~Hz}, 1 \mathrm{H}), 7.89-7.85(\mathrm{~m}, 1 \mathrm{H}), 7.82(\mathrm{ddd}, \mathrm{J}=8.1,7.1,1.2 \mathrm{~Hz}, 1 \mathrm{H})$, $7.66(\mathrm{~d}, \mathrm{~J}=7.4 \mathrm{~Hz}, 1 \mathrm{H}), 7.21(\mathrm{~d}, \mathrm{~J}=1.9 \mathrm{~Hz}, 1 \mathrm{H}), 7.04(\mathrm{~d}, \mathrm{~J}=1.8 \mathrm{~Hz}, 1 \mathrm{H}), 3.74(\mathrm{~s}$, 3H), 2.37 (s, 3H), 2.07 (s, 3H). ${ }^{13} \mathrm{C}$ NMR (126 MHz, Methanol- $\left.d_{4}\right) \delta$ 143.39, 142.82, $138.74,134.18,133.71,133.32,132.73,131.36,130.91,130.89,130.70,130.27$, $128.99,127.16,126.97,126.76,123.39,123.31,117.62,114.38,35.51,20.79,20.72$. HRMS (ESI): $m / z[\mathrm{M}+\mathrm{H}]^{+}$calcd for $\mathrm{C}_{23} \mathrm{H}_{21} \mathrm{~N}_{4}$ 353.1761; found 353.1760. HPLC: $t_{\mathrm{R}}$ $=2.285 \min , 98.88 \%$.

4-(4-(4-((ethylamino)methyl)-2,6-dimethylphenyl)-1-methyl-1H-imidazol-5-yl)-1-na phthonitrile (29). To a solution of $22(50 \mathrm{mg}, 136.8 \mu \mathrm{mol})$ in $\mathrm{CH}_{3} \mathrm{OH}(4 \mathrm{~mL})$ was added and ethylamine aqueous solution $(0.5 \mathrm{~mL})$ and the resulting mixture was stirred at ${ }^{\circ} \mathrm{C}$ for $1 \mathrm{~h}$. Sodium cyanoborohydride $(17 \mathrm{mg}, 273.6 \mu \mathrm{mol})$ was added into the mixture and the reaction mixture was then stirred at $0{ }^{\circ} \mathrm{C}$ for $5 \mathrm{~h}$. Subsequently, the reaction was quenched by saturated aqueous $\mathrm{NaHCO}_{3}$, and was extracted with DCM $\mathrm{MeOH}(5: 1,3 \times 5 \mathrm{~mL})$. The combined organic phase was washed with brine $(10 \mathrm{~mL})$ and dried over anhydrous $\mathrm{Na}_{2} \mathrm{SO}_{4}$, filtered, and concentrated in vacuo. The residue was purified by column chromatography on silica gel (20:1, DCM - MeOH) to obtain the desired product 29. White amorphous solid (14 mg, 26.7 \%). ${ }^{1} \mathrm{H}$ NMR (400 MHz, Methanol- $\left.d_{4}\right) \delta 9.39(\mathrm{~s}, 1 \mathrm{H}), 8.30(\mathrm{~d}, \mathrm{~J}=8.2 \mathrm{~Hz}, 1 \mathrm{H}), 8.05(\mathrm{~d}, \mathrm{~J}=7.4 \mathrm{~Hz}, 1 \mathrm{H}), 7.95(\mathrm{~d}$, $\mathrm{J}=8.2 \mathrm{~Hz}, 1 \mathrm{H}), 7.87(\mathrm{t}, \mathrm{J}=7.2 \mathrm{~Hz}, 1 \mathrm{H}), 7.84-7.77(\mathrm{~m}, 1 \mathrm{H}), 7.65(\mathrm{~d}, \mathrm{~J}=7.4 \mathrm{~Hz}, 1 \mathrm{H})$, $7.31(\mathrm{~s}, 1 \mathrm{H}), 7.16(\mathrm{~s}, 1 \mathrm{H}), 4.07(\mathrm{~s}, 2 \mathrm{H}), 3.73(\mathrm{~s}, 3 \mathrm{H}), 3.05(\mathrm{q}, \mathrm{J}=7.3 \mathrm{~Hz}, 2 \mathrm{H}), 2.34$ (s, 3H), $2.06(\mathrm{~s}, 3 \mathrm{H}), 1.29(\mathrm{t}, \mathrm{J}=7.3 \mathrm{~Hz}, 3 \mathrm{H}) .{ }^{13} \mathrm{C}$ NMR $\left(151 \mathrm{MHz}\right.$, Methanol- $\left.d_{4}\right) \delta$ 
$141.68,141.16,138.56,135.10,133.67,133.28,132.80,131.18,131.03,130.91$, $130.87,130.63,130.32,130.23,129.30,127.74,126.90,126.85,117.62,114.20$, 51.28, 44.05, 35.43, 20.72, 20.66, 11.42. HRMS (ESI): $m / z[\mathrm{M}+\mathrm{H}]^{+}$calcd for $\mathrm{C}_{26} \mathrm{H}_{27} \mathrm{~N}_{4}$ 395.2230; found 395.2239. HPLC: $t_{\mathrm{R}}=2.373$ min, $100 \%$.

The target compounds $\mathbf{3 0}-\mathbf{3 6}, \mathbf{3 9}-\mathbf{4 0}$ and $\mathbf{7 2}$ were prepared from $\mathbf{2 2}$ by following a similar method as described for compound 29. Subsequently, the target compounds 37 - 38 and 41 were prepared from 37 - 38 and $\mathbf{7 2}$ by following a similar method as described for compound $\mathbf{1 3}$.

4-(4-(4-((cyclopropylamino)methyl)-2,6-dimethylphenyl)-1-methyl-1H-imidazol-5-y l)-1-naphthonitrile (30). White amorphous solid (25 mg, 44.9\%). ${ }^{1} \mathrm{H}$ NMR (400 MHz, Methanol- $\left.d_{4}\right) \delta 9.42(\mathrm{~s}, 1 \mathrm{H}), 8.29(\mathrm{~d}, \mathrm{~J}=8.2 \mathrm{~Hz}, 1 \mathrm{H}), 8.05(\mathrm{~d}, \mathrm{~J}=7.4 \mathrm{~Hz}, 1 \mathrm{H}), 7.95(\mathrm{~d}$, $\mathrm{J}=8.2 \mathrm{~Hz}, 1 \mathrm{H}), 7.86(\mathrm{t}, \mathrm{J}=7.4 \mathrm{~Hz}, 1 \mathrm{H}), 7.83-7.78(\mathrm{~m}, 1 \mathrm{H}), 7.65(\mathrm{~d}, \mathrm{~J}=7.4 \mathrm{~Hz}, 1 \mathrm{H})$, $7.32(\mathrm{~s}, 1 \mathrm{H}), 7.17(\mathrm{~s}, 1 \mathrm{H}), 4.20(\mathrm{~s}, 2 \mathrm{H}), 3.74(\mathrm{~s}, 3 \mathrm{H}), 2.71(\mathrm{p}, \mathrm{J}=5.5 \mathrm{~Hz}, 1 \mathrm{H}), 2.34(\mathrm{~s}$, 3H), $2.06(\mathrm{~s}, 3 \mathrm{H}), 0.84(\mathrm{~d}, \mathrm{~J}=5.4 \mathrm{~Hz}, 4 \mathrm{H}) \cdot{ }^{13} \mathrm{C}$ NMR $\left(151 \mathrm{MHz}\right.$, Methanol- $\left.d_{4}\right) \delta$ $141.66,141.12,138.55,134.87,133.66,133.27,132.78,131.06,131.01,130.95$, $130.87,130.65,130.54,130.45,129.21,127.62,126.89,126.86,117.60,114.22$, 52.32, 35.52, 31.28, 20.73, 20.66, 4.19(*2C). HRMS (ESI): $\mathrm{m} / z[\mathrm{M}+\mathrm{H}]^{+}$calcd for $\mathrm{C}_{27} \mathrm{H}_{27} \mathrm{~N}_{4}$ 407.2230; found 407.2235. HPLC: $t_{\mathrm{R}}=2.394$ min, $100 \%$. 4-(4-(4-((tert-butylamino)methyl)-2,6-dimethylphenyl)-1-methyl-1H-imidazol-5-yl)-

1-naphthonitrile (31). White amorphous solid (27 mg, 47.6 \%). ${ }^{1} \mathrm{H}$ NMR (500 MHz, Methanol- $\left.d_{4}\right) \delta 8.23(\mathrm{~d}, \mathrm{~J}=8.3 \mathrm{~Hz}, 1 \mathrm{H}), 8.04(\mathrm{~s}, 1 \mathrm{H}), 7.91(\mathrm{~d}, \mathrm{~J}=7.4 \mathrm{~Hz}, 1 \mathrm{H}), 7.83-$ $7.73(\mathrm{~m}, 2 \mathrm{H}), 7.69$ (ddd, J = 8.3, 7.0, $1.2 \mathrm{~Hz}, 1 \mathrm{H}), 7.41(\mathrm{~d}, \mathrm{~J}=7.4 \mathrm{~Hz}, 1 \mathrm{H}), 7.02(\mathrm{~s}$, 
1H), $6.86(\mathrm{~s}, 1 \mathrm{H}), 3.61(\mathrm{~s}, 2 \mathrm{H}), 3.51(\mathrm{~s}, 3 \mathrm{H}), 2.22(\mathrm{~s}, 3 \mathrm{H}), 1.90(\mathrm{~s}, 3 \mathrm{H}), 1.17$ (s, 9H). ${ }^{13}$ C NMR (126 MHz, Methanol- $\left.d_{4}\right) \delta$ 139.00, 138.75, 138.67, 138.13, 132.89, 132.39, $132.10,131.88,131.85(* 2 \mathrm{C}), 128.80,128.41,128.14(* 2 \mathrm{C}), 127.41,127.38,126.23$, $125.12,116.73,110.64,51.72,45.85,31.72,26.67(* 3 C), 19.53,19.41$. HRMS (ESI): $m / z[\mathrm{M}+\mathrm{H}]^{+}$calcd for $\mathrm{C}_{28} \mathrm{H}_{31} \mathrm{~N}_{4}$ 423.2543; found 423.2544. HPLC: $t_{\mathrm{R}}=2.488 \mathrm{~min}$, $97.20 \%$.

4-(4-(2,6-dimethyl-4-(pyrrolidin-1-ylmethyl)phenyl)-1-methyl-1H-imidazol-5-yl)-1naphthonitrile (32). White amorphous solid (25 mg, $44.3 \%) .{ }^{1} \mathrm{H}$ NMR (600 MHz, Methanol- $\left.d_{4}\right) \delta 8.21(\mathrm{~d}, \mathrm{~J}=8.3 \mathrm{~Hz}, 1 \mathrm{H}), 8.04(\mathrm{~s}, 1 \mathrm{H}), 7.91(\mathrm{~d}, \mathrm{~J}=7.4 \mathrm{~Hz}, 1 \mathrm{H}), 7.79(\mathrm{~d}$, $\mathrm{J}=8.4 \mathrm{~Hz}, 1 \mathrm{H}), 7.74(\mathrm{t}, \mathrm{J}=7.5 \mathrm{~Hz}, 1 \mathrm{H}), 7.67(\mathrm{t}, \mathrm{J}=7.6 \mathrm{~Hz}, 1 \mathrm{H}), 7.41(\mathrm{~d}, \mathrm{~J}=7.4 \mathrm{~Hz}$ 1H), $6.98(\mathrm{~s}, 1 \mathrm{H}), 6.83(\mathrm{~s}, 1 \mathrm{H}), 3.51(\mathrm{~s}, 3 \mathrm{H}), 3.45(\mathrm{~s}, 2 \mathrm{H}), 2.44(\mathrm{~s}, 4 \mathrm{H}), 2.18(\mathrm{~s}, 3 \mathrm{H})$, $1.90(\mathrm{~s}, 3 \mathrm{H}), 1.74(\mathrm{~s}, 4 \mathrm{H}) .{ }^{13} \mathrm{C}$ NMR (151 MHz, Methanol- $\left.d_{4}\right) \delta$ 140.43, 140.17, $139.81,139.16,138.95,134.26,133.80,133.42,133.27,133.15,130.12,129.75$, $129.44,129.37(* 2 \mathrm{C}), 128.79,127.65,126.51,118.16,111.99,61.11,54.85(* 2 \mathrm{C})$, 33.16, 23.99(*2C), 20.94, 20.85. HRMS (ESI): $m / z[\mathrm{M}+\mathrm{H}]^{+}$calcd for $\mathrm{C}_{28} \mathrm{H}_{29} \mathrm{~N}_{4}$ 421.2387; found 421.2388. HPLC: $t_{\mathrm{R}}=2.379 \mathrm{~min}, 96.07 \%$.

4-(4-(2,6-dimethyl-4-(piperidin-1-ylmethyl)phenyl)-1-methyl-1H-imidazol-5-yl)-1-n aphthonitrile (33). White amorphous solid (25 mg, 42.1\%). ${ }^{1} \mathrm{H}$ NMR (600 MHz, Methanol- $\left.d_{4}\right) \delta 8.22(\mathrm{~d}, \mathrm{~J}=8.3 \mathrm{~Hz}, 1 \mathrm{H}), 8.04(\mathrm{~s}, 1 \mathrm{H}), 7.92(\mathrm{~d}, \mathrm{~J}=7.4 \mathrm{~Hz}, 1 \mathrm{H}), 7.79(\mathrm{~d}$, $\mathrm{J}=8.4 \mathrm{~Hz}, 1 \mathrm{H}), 7.75(\mathrm{ddd}, \mathrm{J}=8.2,7.0,1.1 \mathrm{~Hz}, 1 \mathrm{H}), 7.67(\mathrm{ddd}, \mathrm{J}=8.2,7.0,1.1 \mathrm{~Hz}$ 1H), $7.41(\mathrm{~d}, \mathrm{~J}=7.4 \mathrm{~Hz}, 1 \mathrm{H}), 6.96(\mathrm{~s}, 1 \mathrm{H}), 6.82(\mathrm{~s}, 1 \mathrm{H}), 3.52(\mathrm{~s}, 3 \mathrm{H}), 3.32(\mathrm{~s}, 2 \mathrm{H})$, $2.17(\mathrm{~s}, 3 \mathrm{H}), 1.90(\mathrm{~s}, 3 \mathrm{H}), 1.53(\mathrm{p}, \mathrm{J}=5.6 \mathrm{~Hz}, 4 \mathrm{H}), 1.20-1.18(\mathrm{~m}, 6 \mathrm{H}) .{ }^{13} \mathrm{C} \mathrm{NMR}$ 
$\left(151 \mathrm{MHz}\right.$, Methanol- $\left.d_{4}\right) \delta 139.05,138.79,138.28,137.64,136.28,132.86,132.42$, $132.08,131.87,131.74,129.48,128.72,128.57,128.34,128.02,127.40,126.26$, $125.11,116.77,110.60,74.43,62.97(* 2 \mathrm{C}), 31.77,24.87(* 2 \mathrm{C}), 23.63(* 2 \mathrm{C}), 19.52$. HRMS (ESI): $m / z[\mathrm{M}+\mathrm{H}]^{+}$calcd for $\mathrm{C}_{29} \mathrm{H}_{31} \mathrm{~N}_{4}$ 435.2543; found 435.2549. HPLC: $t_{\mathrm{R}}$ $=2.440 \min , 100 \%$.

4-(4-(2,6-dimethyl-4-(morpholinomethyl)phenyl)-1-methyl-1H-imidazol-5-yl)-1-nap hthonitrile (34). White amorphous solid (30 mg, $50.5 \%) .{ }^{1} \mathrm{H}$ NMR (400 MHz, Methanol-d $\left.d_{4}\right) \delta 9.39(\mathrm{~s}, 1 \mathrm{H}), 8.30(\mathrm{~d}, \mathrm{~J}=8.2 \mathrm{~Hz}, 1 \mathrm{H}), 8.06(\mathrm{~d}, \mathrm{~J}=7.4 \mathrm{~Hz}, 1 \mathrm{H}), 7.94(\mathrm{~d}$, $\mathrm{J}=8.2 \mathrm{~Hz}, 1 \mathrm{H}), 7.86(\mathrm{t}, \mathrm{J}=7.4 \mathrm{~Hz}, 1 \mathrm{H}), 7.85-7.77(\mathrm{~m}, 1 \mathrm{H}), 7.65(\mathrm{~d}, \mathrm{~J}=7.4 \mathrm{~Hz}, 1 \mathrm{H})$, $7.40(\mathrm{~s}, 1 \mathrm{H}), 7.25(\mathrm{~s}, 1 \mathrm{H}), 4.26(\mathrm{~s}, 2 \mathrm{H}), 3.98(\mathrm{~d}, \mathrm{~J}=11.4 \mathrm{~Hz}, 2 \mathrm{H}), 3.78$ (d, J = $10.8 \mathrm{~Hz}$, 2H), $3.73(\mathrm{~s}, 3 \mathrm{H}), 3.25(\mathrm{~s}, 2 \mathrm{H}), 3.16(\mathrm{~d}, \mathrm{~J}=11.5 \mathrm{~Hz}, 2 \mathrm{H}), 2.35(\mathrm{~s}, 3 \mathrm{H}), 2.06(\mathrm{~s}, 3 \mathrm{H})$.

${ }^{13} \mathrm{C}$ NMR $\left(126 \mathrm{MHz}\right.$, Methanol- $\left.d_{4}\right) \delta 141.83,141.29,138.66,133.72,133.36,132.74$, $132.04,131.98,131.91,131.09,131.01,130.91,130.85,130.62,129.23,128.53$, $126.94,126.82,117.64,114.28,64.77(* 2 \mathrm{C}), 61.18,52.94(* 2 \mathrm{C}), 35.44,20.71,20.68$. HRMS (ESI): $m / z[\mathrm{M}+\mathrm{H}]^{+}$calcd for $\mathrm{C}_{28} \mathrm{H}_{29} \mathrm{~N}_{4} \mathrm{O}$ 437.2336; found 437.2339. HPLC: $t_{\mathrm{R}}=2.394 \min , 99.07 \%$.

tert-butyl

4-(4-(5-(4-cyanonaphthalen-1-yl)-1-methyl-1H-imidazol-4-yl)-3,5-dimethylbenzyl)pip erazine-1-carboxylate (35). White amorphous solid (30 mg, 40.9\%). ${ }^{1} \mathrm{H}$ NMR (400 MHz, Chloroform- $d$ ) $\delta 8.27(\mathrm{dd}, \mathrm{J}=8.3,1.4 \mathrm{~Hz}, 1 \mathrm{H}), 7.88(\mathrm{~s}, 1 \mathrm{H}), 7.79(\mathrm{~d}, \mathrm{~J}=7.4 \mathrm{~Hz}$, 1H), $7.73-7.65(\mathrm{~m}, 2 \mathrm{H}), 7.60(\mathrm{ddd}, \mathrm{J}=8.4,7.0,1.3 \mathrm{~Hz}, 1 \mathrm{H}), 7.25(\mathrm{~d}, \mathrm{~J}=7.4 \mathrm{~Hz}, 1 \mathrm{H})$, $6.95-6.90(\mathrm{~m}, 1 \mathrm{H}), 6.74(\mathrm{~d}, \mathrm{~J}=1.7 \mathrm{~Hz}, 1 \mathrm{H}), 3.43(\mathrm{~s}, 3 \mathrm{H}), 3.39(\mathrm{~m}, 4 \mathrm{H}), 3.35(\mathrm{~s}, 2 \mathrm{H})$, 
$2.35-2.30(\mathrm{~m}, 4 \mathrm{H}), 2.20(\mathrm{~s}, 3 \mathrm{H}), 1.81(\mathrm{~s}, 3 \mathrm{H}), 1.43(\mathrm{~s}, 9 \mathrm{H}) .{ }^{13} \mathrm{C}$ NMR $(151 \mathrm{MHz}$, Chloroform-d) $\delta 174.65,154.84,139.70,138.98,138.47,137.83,136.47,133.13$, $132.79,132.09,131.86,128.84,128.38(* 2 \mathrm{C}), 128.32,128.28,127.32,126.25,126.07$, $117.52,111.15,79.71,62.73(* 2 C), 52.78,32.96,28.51(* 3 C), 20.92,20.71$. HRMS (ESI): $m / z[\mathrm{M}+\mathrm{H}]^{+}$calcd for $\mathrm{C}_{33} \mathrm{H}_{38} \mathrm{~N}_{5} \mathrm{O}_{2}$ 536.3020; found 536.3015. HPLC: $t_{\mathrm{R}}=$ $2.699 \min , 98.48 \%$.

tert-butyl

(1-(4-(5-(4-cyanonaphthalen-1-yl)-1-methyl-1H-imidazol-4-yl)-3,5-dimethylbenzyl)pi peridin-4-yl)carbamate (36). White amorphous solid (34 mg, 45.1\%). ${ }^{1} \mathrm{H}$ NMR (600 MHz, Chloroform- $d$ ) $\delta 8.27(\mathrm{~d}, \mathrm{~J}=8.3 \mathrm{~Hz}, 1 \mathrm{H}), 7.82-7.76(\mathrm{~m}, 2 \mathrm{H}), 7.75-7.67(\mathrm{~m}$, 2H), $7.62-7.57(\mathrm{~m}, 1 \mathrm{H}), 7.25(\mathrm{~d}, \mathrm{~J}=8.0 \mathrm{~Hz}, 1 \mathrm{H}), 6.92(\mathrm{~s}, 1 \mathrm{H}), 6.74(\mathrm{~s}, 1 \mathrm{H}), 4.47-$ $4.37(\mathrm{~m}, 1 \mathrm{H}), 3.43(\mathrm{~s}, 3 \mathrm{H}), 3.31(\mathrm{~s}, 2 \mathrm{H}), 2.79-2.65(\mathrm{~m}, 2 \mathrm{H}), 2.27-2.14(\mathrm{~m}, 4 \mathrm{H})$, $2.06-1.96(\mathrm{~m}, 4 \mathrm{H}), 1.86(\mathrm{~d}, \mathrm{~J}=11.3 \mathrm{~Hz}, 2 \mathrm{H}), 1.82(\mathrm{~s}, 3 \mathrm{H}), 1.43(\mathrm{~s}, 9 \mathrm{H}) .{ }^{13} \mathrm{C}$ NMR (151 MHz, Chloroform-d) $\delta 175.39,155.11,140.13,138.70,138.21,137.55,133.26$, $132.63,131.93,131.71,129.94,129.66,128.61,128.11,128.06,128.03,127.07$, $126.16,125.88,117.41,110.82,79.13,62.79,52.29,35.83,32.69,28.34(* 3 C), 25.45$, 22.60, 20.76, 20.53, 14.04. HRMS (ESI): $m / z[\mathrm{M}+\mathrm{H}]^{+}$calcd for $\mathrm{C}_{34} \mathrm{H}_{40} \mathrm{~N}_{5} \mathrm{O}_{2}$ 550.3177; found 550.3170. HPLC: $t_{\mathrm{R}}=2.680 \mathrm{~min}, 100 \%$.

4-(4-(2,6-dimethyl-4-(piperazin-1-ylmethyl)phenyl)-1-methyl-1H-imidazol-5-yl)-1-n aphthonitrile (37). White amorphous solid (10 mg, 85\%). ${ }^{1} \mathrm{H}$ NMR (400 MHz, Methanol- $\left.d_{4}\right) \delta 9.42(\mathrm{~s}, 1 \mathrm{H}), 8.30(\mathrm{~d}, \mathrm{~J}=8.1 \mathrm{~Hz}, 1 \mathrm{H}), 8.07(\mathrm{~d}, \mathrm{~J}=7.3 \mathrm{~Hz}, 1 \mathrm{H}), 7.95(\mathrm{~d}$, $\mathrm{J}=8.2 \mathrm{~Hz}, 1 \mathrm{H}), 7.87(\mathrm{t}, \mathrm{J}=7.2 \mathrm{~Hz}, 1 \mathrm{H}), 7.85-7.79(\mathrm{~m}, 1 \mathrm{H}), 7.65(\mathrm{~d}, \mathrm{~J}=7.4 \mathrm{~Hz}, 1 \mathrm{H})$, 
$7.47(\mathrm{~s}, 1 \mathrm{H}), 7.31(\mathrm{~s}, 1 \mathrm{H}), 4.40(\mathrm{~s}, 2 \mathrm{H}), 3.72(\mathrm{~s}, 3 \mathrm{H}), 3.65-3.49(\mathrm{~m}, 8 \mathrm{H}), 2.37(\mathrm{~s}, 3 \mathrm{H})$, $2.05(\mathrm{~s}, 3 \mathrm{H}) .{ }^{13} \mathrm{C}$ NMR $\left(151 \mathrm{MHz}\right.$, Methanol- $\left.d_{4}\right) \delta 141.96,141.40,138.60,133.71$, $133.44,132.74,132.10,131.98,131.75,131.12,130.96,130.86,130.77,130.68$, $129.08,128.52,126.97,126.80,117.66,114.32,60.84,49.28(* 2 \mathrm{C}), 41.85(* 2 \mathrm{C}), 35.46$, 20.71, 20.66. HRMS (ESI): $m / z[M+H]^{+}$calcd for $\mathrm{C}_{28} \mathrm{H}_{30} \mathrm{~N}_{5}$ 436.2496; found 436.2499. HPLC: $t_{\mathrm{R}}=2.225 \min , 97.66 \%$.

4-(4-(4-((4-aminopiperidin-1-yl)methyl)-2,6-dimethylphenyl)-1-methyl-1H-imidazol -5-yl)-1-naphthonitrile (38). White amorphous solid (11 mg, 88\%). ${ }^{1} \mathrm{H}$ NMR (600 MHz, Methanol- $\left.d_{4}\right) \delta 8.21(\mathrm{~d}, \mathrm{~J}=8.3 \mathrm{~Hz}, 1 \mathrm{H}), 8.04(\mathrm{~s}, 1 \mathrm{H}), 7.90(\mathrm{dd}, \mathrm{J}=8.6,4.4 \mathrm{~Hz}$, 1H), $7.79(\mathrm{~d}, \mathrm{~J}=8.4 \mathrm{~Hz}, 1 \mathrm{H}), 7.76-7.72(\mathrm{~m}, 1 \mathrm{H}), 7.68-7.63(\mathrm{~m}, 1 \mathrm{H}), 7.41(\mathrm{~d}, \mathrm{~J}=$ $7.4 \mathrm{~Hz}, 1 \mathrm{H}), 6.95(\mathrm{~s}, 1 \mathrm{H}), 6.81(\mathrm{~s}, 1 \mathrm{H}), 3.52(\mathrm{~s}, 3 \mathrm{H}), 3.31(\mathrm{~s}, 2 \mathrm{H}), 2.77-2.70(\mathrm{~m}, 2 \mathrm{H})$, $2.56(\mathrm{dt}, \mathrm{J}=10.7,6.6 \mathrm{~Hz}, 1 \mathrm{H}), 2.17(\mathrm{~s}, 3 \mathrm{H}), 1.95(\mathrm{t}, \mathrm{J}=11.6 \mathrm{~Hz}, 2 \mathrm{H}), 1.90(\mathrm{~s}, 3 \mathrm{H})$, $1.76-1.72(\mathrm{~m}, 2 \mathrm{H}), 1.38-1.29(\mathrm{~m}, 2 \mathrm{H}) .{ }^{13} \mathrm{C}$ NMR $\left(126 \mathrm{MHz}\right.$, Methanol- $\left.d_{4}\right) \delta 141.79$ 141.24, 138.66, 133.66, 133.43, 132.69, 132.43, 131.97, 131.88, 131.04, 131.01, $130.88, \quad 130.83,130.63,129.16, \quad 128.33,126.87(* 2 \mathrm{C}), 117.66,114.18,60.82$, 51.70(*2C), 46.82, 35.64, 28.24(*2C), 20.74(*2C). HRMS (ESI): $m / z[\mathrm{M}+\mathrm{H}]^{+}$calcd for $\mathrm{C}_{29} \mathrm{H}_{32} \mathrm{~N}_{5}$ 450.2652; found 450.2656. HPLC: $t_{\mathrm{R}}=2.199 \mathrm{~min}, 99.25 \%$.

4-(4-(4-((3-hydroxypyrrolidin-1-yl)methyl)-2,6-dimethylphenyl)-1-methyl-1H-imida zol-5-yl)-1-naphthonitrile (39). White amorphous solid (22 mg, 37.2\%). ${ }^{1} \mathrm{H}$ NMR $\left(400 \mathrm{MHz}\right.$, Methanol- $\left.d_{4}\right) \delta 8.23(\mathrm{~d}, \mathrm{~J}=8.3 \mathrm{~Hz}, 1 \mathrm{H}), 8.04(\mathrm{~s}, 1 \mathrm{H}), 7.93(\mathrm{~d}, \mathrm{~J}=7.4 \mathrm{~Hz}$, 1H), $7.78(\mathrm{q}, \mathrm{J}=8.0,7.4 \mathrm{~Hz}, 2 \mathrm{H}), 7.71-7.64(\mathrm{~m}, 1 \mathrm{H}), 7.41(\mathrm{~d}, \mathrm{~J}=7.4 \mathrm{~Hz}, 1 \mathrm{H}), 6.99$ $(\mathrm{s}, 1 \mathrm{H}), 6.84(\mathrm{~s}, 1 \mathrm{H}), 4.28(\mathrm{tt}, \mathrm{J}=6.9,3.3 \mathrm{~Hz}, 1 \mathrm{H}), 3.52(\mathrm{~s}, 3 \mathrm{H}), 3.50-3.41(\mathrm{~m}, 2 \mathrm{H})$, 
$2.71(\mathrm{dt}, \mathrm{J}=10.6,5.5 \mathrm{~Hz}, 1 \mathrm{H}), 2.63(\mathrm{q}, \mathrm{J}=7.7 \mathrm{~Hz}, 1 \mathrm{H}), 2.46(\mathrm{q}, \mathrm{J}=8.5 \mathrm{~Hz}, 1 \mathrm{H}), 2.37$ $(\mathrm{dd}, \mathrm{J}=10.4,3.2 \mathrm{~Hz}, 1 \mathrm{H}), 2.19(\mathrm{~s}, 3 \mathrm{H}), 2.08(\mathrm{dd}, \mathrm{J}=13.6,7.3 \mathrm{~Hz}, 1 \mathrm{H}), 1.88(\mathrm{~s}, 3 \mathrm{H})$, $1.70-1.59(\mathrm{~m}, 1 \mathrm{H}) .{ }^{13} \mathrm{C}$ NMR $\left(126 \mathrm{MHz}\right.$, Methanol- $\left.d_{4}\right) \delta 140.42,140.20,139.83$, $139.16,138.94,134.27,133.83,133.39,133.28,133.17,130.13,129.75,129.45$, $129.23,128.81,127.65,126.53,118.18,112.02,71.42,63.31,61.22,53.68,49.51$, 35.06, 33.15(*2C), 20.92. HRMS (ESI): $m / z[\mathrm{M}+\mathrm{H}]^{+}$calcd for $\mathrm{C}_{28} \mathrm{H}_{29} \mathrm{~N}_{4} \mathrm{O}$ 437.2336; found 437.2338. HPLC: $t_{\mathrm{R}}=2.343 \mathrm{~min}, 100 \%$.

4-(4-(4-((3-(dimethylamino)pyrrolidin-1-yl)methyl)-2,6-dimethylphenyl)-1-methyl-1 H-imidazol-5-yl)-1-naphthonitrile (40). White amorphous solid (20 mg, 31.2\%). ${ }^{1} \mathrm{H}$ NMR (500 MHz, Methanol- $\left.d_{4}\right) \delta 9.40(\mathrm{~s}, 1 \mathrm{H}), 8.31(\mathrm{~d}, \mathrm{~J}=8.2 \mathrm{~Hz}, 1 \mathrm{H}), 8.07(\mathrm{~d}, \mathrm{~J}=$ $7.2 \mathrm{~Hz}, 1 \mathrm{H}), 7.93(\mathrm{~d}, \mathrm{~J}=8.2 \mathrm{~Hz}, 1 \mathrm{H}), 7.87(\mathrm{t}, \mathrm{J}=7.5 \mathrm{~Hz}, 1 \mathrm{H}), 7.82(\mathrm{t}, \mathrm{J}=7.4 \mathrm{~Hz}, 1 \mathrm{H})$, $7.65(\mathrm{~d}, \mathrm{~J}=7.2 \mathrm{~Hz}, 1 \mathrm{H}), 7.44(\mathrm{~s}, 1 \mathrm{H}), 7.28(\mathrm{~s}, 1 \mathrm{H}), 4.43(\mathrm{~s}, 2 \mathrm{H}), 4.23(\mathrm{~s}, 1 \mathrm{H}), 3.88-$ $3.82(\mathrm{~m}, 1 \mathrm{H}), 3.78(\mathrm{~s}, 1 \mathrm{H}), 3.73(\mathrm{~s}, 3 \mathrm{H}), 3.57(\mathrm{~s}, 1 \mathrm{H}), 3.43(\mathrm{~s}, 1 \mathrm{H}), 2.95(\mathrm{~s}, 6 \mathrm{H}), 2.60$ $(\mathrm{s}, 1 \mathrm{H}), 2.43(\mathrm{~s}, 1 \mathrm{H}), 2.35(\mathrm{~s}, 3 \mathrm{H}), 2.05(\mathrm{~s}, 3 \mathrm{H}) .{ }^{13} \mathrm{C}$ NMR $\left(126 \mathrm{MHz}\right.$, Methanol- $\left.d_{4}\right) \delta$ $141.97,141.41,138.61,133.72,133.51,133.40,132.74,131.15,131.12,131.04$, $130.95,130.92,130.87,130.67,129.13,128.32,126.97,126.80,117.65,114.31$, 64.61, 58.93, 54.22, 42.76, 35.50(*2C), 26.90, 23.70, 20.68(*2C). HRMS (ESI): $\mathrm{m} / \mathrm{z}$ $[\mathrm{M}+\mathrm{H}]^{+}$calcd for $\mathrm{C}_{30} \mathrm{H}_{34} \mathrm{~N}_{5}$ 464.2809; found 464.2811. HPLC: $t_{\mathrm{R}}=2.279 \mathrm{~min}$, $100 \%$.

4-(4-(4-(((2-aminoethyl)amino)methyl)-2,6-dimethylphenyl)-1-methyl-1H-imidazol5-yl)-1-naphthonitrile (41). White amorphous solid (10 mg, 80\%). ${ }^{1} \mathrm{H}$ NMR (500 MHz, Methanol- $\left.d_{4}\right) \delta 9.40(\mathrm{~s}, 1 \mathrm{H}), 8.30(\mathrm{~d}, \mathrm{~J}=8.3 \mathrm{~Hz}, 1 \mathrm{H}), 8.05(\mathrm{~d}, \mathrm{~J}=7.0 \mathrm{~Hz}, 1 \mathrm{H})$, 
$7.93(\mathrm{~d}, \mathrm{~J}=8.2 \mathrm{~Hz}, 1 \mathrm{H}), 7.87(\mathrm{t}, \mathrm{J}=7.5 \mathrm{~Hz}, 1 \mathrm{H}), 7.81(\mathrm{t}, \mathrm{J}=7.4 \mathrm{~Hz}, 1 \mathrm{H}), 7.63(\mathrm{~d}, \mathrm{~J}=$ $7.2 \mathrm{~Hz}, 1 \mathrm{H}), 7.40(\mathrm{~s}, 1 \mathrm{H}), 7.24(\mathrm{~s}, 1 \mathrm{H}), 4.19(\mathrm{~s}, 2 \mathrm{H}), 3.72(\mathrm{~s}, 3 \mathrm{H}), 3.41(\mathrm{t}, \mathrm{J}=6.6 \mathrm{~Hz}$, 2H), $3.34(\mathrm{t}, \mathrm{J}=6.3 \mathrm{~Hz}, 2 \mathrm{H}), 2.35(\mathrm{~s}, 3 \mathrm{H}), 2.04(\mathrm{~s}, 3 \mathrm{H}) .{ }^{13} \mathrm{C}$ NMR (126 MHz, Methanol- $\left.d_{4}\right) \delta 141.74,141.20,138.57,134.60,133.69,133.34,132.80,131.10$, $131.03,130.96,130.90,130.69,130.54,130.41,129.15,127.79,126.95,126.83$, 117.61, 114.30, 52.09, 45.66, 36.80, 35.52, 20.74, 20.67. HRMS (ESI): $m / z[\mathrm{M}+\mathrm{H}]^{+}$ calcd for $\mathrm{C}_{26} \mathrm{H}_{28} \mathrm{~N}_{5}$ 410.2339; found 410.2337. HPLC: $t_{\mathrm{R}}=2.172 \mathrm{~min}, 100 \%$.

Protein Expression and Purification. DNA fragment encoding amino acids 211 to 350 of NSD2-PWWP1 subcloned in the pET-28 plasmid was produced by Shanghai Generay Biotech Co. Ltd. The constructed plasmid containing six histidine or sumo tags in the N-terminus was transformed into Escherichia coli BL21(DE3) cell with heat shock. Transformed E. coli cells were grown to an OD600 of $1.2-1.5$ at $37{ }^{\circ} \mathrm{C}$ in TB medium containing $50 \mathrm{mg} / \mathrm{L}$ kanamycin. Then, the culture temperature was reduced to $18{ }^{\circ} \mathrm{C}$ and the cultures were then induced with $0.5 \mathrm{mM}$ IPTG for $16 \mathrm{~h}$. The bacterial cells were harvested after centrifugation, washed with PBS for 3 times and frozen at $-80{ }^{\circ} \mathrm{C}$. Harvested bacterial cells were lysed with ultra-high pressure cell disrupter (UH-03, Union, Shanghai, China) in lysis buffer (50 mM Tris, $\mathrm{pH}$ 8.0, 50 $\mathrm{mM} \mathrm{NaCl}, 5 \%$ glycerol, and $0.5 \mathrm{mM}$ TCEP). The supernatant was collected after centrifugation at $12000 \mathrm{rpm}$ for 60min. Subsequently, Ni Sepherose was added to the supernatant to bind to the protein, and the target protein was eluted with a buffer containing $250 \mathrm{mM}$ imidazole. The target protein was concentrated and purified with exclusion chromatography (Superdex 200 10/300 GL124mL) which was balanced by 
buffer (50 mM HEPES, $50 \mathrm{mM} \mathrm{NaCl}, 5 \%$ glycerol, pH 8.0 and $0.5 \mathrm{mM}$ TCEP). Purified NSD2-PWWP1 protein concentrated to $10 \mathrm{mg} / \mathrm{ml}$ with approximately $95 \%$ purity was either used to set up crystals or flash frozen in liquid nitrogen for storage at $-78^{\circ} \mathrm{C}$.

Crystallization and X-ray Crystallography. The vapor diffusion method was used for crystallization of aliquots of the purified proteins. The protein of NSD2-PWWP1, and the ligands were grown at $16{ }^{\circ} \mathrm{C}$ in $1 \mu \mathrm{L}$ of protein with an equal volume of reservoir solution containing the $1.6 \mathrm{M}$ ammonium sulfate, $0.01 \mathrm{M}$ magnesium chloride, 0.1 M HEPES at pH 7.5. Crystals grew to diffracting quality within 1 week. Data was collected at $100 \mathrm{~K}$ on beam line BL17U at the Shanghai Synchrotron Radiation Facility (SSRF) (Shanghai, China) for the co-crystallized structures. ${ }^{16}$ The data were processed with the XDS software packages ${ }^{17}$, and the structures were then solved by molecular replacement using the PHENIX software package. ${ }^{18}$ The search model used for the crystals was the NSD2-PWWP1 complex structure (PDB code 6UE6). The structures were refined using the CCP4 program REFMAC5 ${ }^{19}$ combined with the simulated annealing protocol implemented in the program PHENIX. With the aid of the program $\operatorname{Coot}^{20}$, compound, water molecules, and others were fitted into the initial $F_{\mathrm{o}}-F_{\mathrm{c}}$ maps.

Enzymatic Assays for NSD2-PWWP1. The enzyme activity was determined by HTRF assay which was conducted according to the manufacturer's protocol (Cisbio) in a 96-well plate. First, $5 \mu \mathrm{L}$ of 4 nM NSD2 PWWP1 diluent buffer and $5 \mu \mathrm{L}$ of the substrate substrate polypeptide diluent buffer was added to each well, then $5 \mu \mathrm{L}$ of the 
corresponding concentration of compounds dissolved in diluent buffer, finally, $5 \mu \mathrm{L}$ of $\mathrm{Tb} / \mathrm{d} 2$ mixture were then added. After incubated at room temperature for $3 \mathrm{~h}$, the plate was read on a microplate reader (BioTek Cytation 5) at 665 and $620 \mathrm{~nm}$. HTRF signals were calculated as a ratio as follows: (intensity of $665 \mathrm{~nm}$ )/(intensity of 620 $\mathrm{nm}) \times 10^{4}$. All compounds were dissolved in DMSO and added to the medium at a final 1:1000 dilution. All experiments were repeated at least twice. The enzyme activity calculation formula was as follows: Inhibition= (maximum intensity for complete binding of protein and peptide- intensity after adding the inhibitor )/(maximum intensity for complete binding of protein and peptidebackground values of intensity only containing $\mathrm{Tb}$ and $\mathrm{d} 2) \times 100 \%$. The calculation of compounds $\mathrm{IC}_{50}$ took the logarithm of compound concentration as the abscissa, and the corresponding inhibition ratio as the ordinate, and nonlinear fitting was performed. The reagents and equipment used in this experiment were as follows: the fluorescent donor was Anti-His $\mathrm{Tb}$ (Cisbio,PerkinElmer), the fluorescent receptor was Streptavidin-d2, the protein was His-NSD2-PWWP1(211-350aa) purified in our laboratory, and the substrate polypeptide was Biotin-ATKAARKSAPATGGV-Lys (me) $)_{2}$-KPHRYRPG (Shanghai Desheng Pharmaceutical Technology Co., Ltd), buffer used from Cisbio Perkinelmer.

Thermal Shift Assay. Melting curves of proteins with and without the presence of compounds were determined by TSA using the Protein thermal shift ${ }^{\mathrm{TM}}$ Dye Kit (Life Technologies). The kit containing Protein Thermal Shift ${ }^{\mathrm{TM}}$ Buffer and Protein Thermal Shift ${ }^{\mathrm{TM}}$ Dye. The reaction system contained 5ul Protein Thermal Shift ${ }^{\mathrm{TM}}$ 
Buffer, 2.5ul Diluted Protein Thermal Shift ${ }^{\mathrm{TM}}$ Dye $(8 \times)$ and $12.5 \mathrm{ul}$ Water + protein + buffer and/or buffer components. The melting curve was generated by heating the plate with a slope of $2.5 \mathrm{oC} / \mathrm{min}$ from $25{ }^{\circ} \mathrm{C}$ to $95{ }^{\circ} \mathrm{C}$. The assay was performed on QuantStudioTM 6 Flex real-time PCR machine (Applied Biosystems) and analyzed

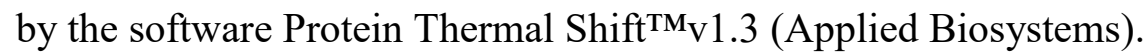

AlphaLISA NSD2 Histone H3 Lysine-N-methyltransferase Assay. AlphaLISA NSD2 Histone H3 Lysine-N-methyltransferase assay were used to determined the $\mathrm{IC}_{50}$ of compound 9c. The enzyme prep was diluted in assay buffer before use. SAM (TAKA) was used as the methyl group donor and unmethylated histone H3 (Anaspec) was used as a substrate. All these components were incubated at room temperature (r.t.) for 20 min. Add $5 \mu \mathrm{L}$ of high salt buffer to stop the NSD2 enzymatic reaction. Cover the plate with TopSeal-A film and incubate $30 \mathrm{~min}$ at room temperature (r.t.) acceptor beads, biotinylatedanti-H3 antibody and donor beads were then diluted in $1 \mathrm{X}$ detection buffer (PerkinElmer) before use. Acceptor beads/biotinylated antibody mix were then added at a final concentration of $20 \mu \mathrm{g} / \mathrm{mL}$ and $1 \mathrm{nM}$ to the reaction mixture and the plate was incubated at r.t. for $1 \mathrm{~h}$. Donor beads were added at a final concentration of $20 \mu \mathrm{g} / \mathrm{mL}$ and the plate was incubated at r.t. for $30 \mathrm{~min}$ in subdued light. The reaction was run in 384-well plates. The plates were read using an EnVision® reader. The resultant data were analyzed with graphPad prism.

Western Blot analysis. Total cellular extracts were lysed in buffer containing $2 \%$ SDS and quantified by the method of BCA protein assay. An equal amount of protein was subjected to electrophoresis on SDS-PAGE and transferred to nitrocellulose membranes, incubated with primary antibodies and then incubated with secondary antibody at room temperature for $1 \mathrm{~h}$. Primary antibodies used were as follows: anti-beta-actin(Proteintech Group, 66009-1-lg), anti-histone H3 (Cell Signaling 
Technology, \#9715), anti-WHSC1/NSD2(abcam, ab75359), anti-Di-Methyl-Histone H3 (Lys36) (Cell Signaling Technology, \#2901). The detection was performed using a SuperSignal west pico stable peroxide solution (Thermo Fisher Scientific).

Cell Proliferation Assay. The cell lines used in each experiment were obtained from the American Type Culture Collection. The cell line RS4:11, and KMS11 were cultured in the altered RPMI-1640 medium (Invitrogen) supplemented with 10\% FBS (Gibco) and $2 \mathrm{mM}$ lglutamine. The MV4:11 cell line was maintained in IMDM (Iscove's Modified Dulbecco's Medium) supplemented with 10\% selected FBS (Gibco). For the cell proliferation assay, Cells were plated in 96-well plates at 6000-20000 cells per well in a volume of $200 \mu \mathrm{L}$ for $24 \mathrm{~h}$. Then different concentrations of diluted compounds or DMSO added into the the cells and were cultured for another $6 \mathrm{~d}$. Dosages corresponding to the half maximal inhibition $\left(\mathrm{IC}_{50}\right)$ were detected using the cell counting kit-8 (Life Technologies) according to the manufacturer's instructions.

Cell Cycle Analysis and Annexin V Staining. Cell cycle analysis was conducted by incorporating propidium iodide (PI). The cells were treated with $10 \mathrm{uM}, 5 \mathrm{uM} 033$ or DMSO control for $48 \mathrm{~h}$. Then cells were harvested and resuspended in PBS and fixed by $70 \%$ pre-cooled ethanol overnight. Then Cells were washed with PBS and resuspended in stain buffer containing $5 \mu \mathrm{g} / \mathrm{mL}$ ribonuclease A (Sigma-Aldrich) and 5 $\mu \mathrm{g} / \mathrm{mL}$ PI for $45 \mathrm{~min}$ in room tempreture for $40 \mathrm{~min}$. Acquisition was immediately performed on FACSCanto (BD Biosciences), and results were analyzed using modifit software.

Annexin V binding assay was used for apoptosis assay. The cells were cultured in 6 well plates at the optimal density. After 24h, different concentrations of the test compounds or DMSO control was added in the 
plates for another $72 \mathrm{~h}$. Then, $10^{5}$ cells were collected and re-suspended in $100 \mu \mathrm{L}$ annexin $\mathrm{V}$ binding solution containing $0.25 \mu \mathrm{g} / \mathrm{mL}$ anti-annexin $\mathrm{V}$-FITC and $1 \mu \mathrm{g} / \mathrm{mL}$ PI in the dark for 10 min. Samples were diluted with $400 \mu \mathrm{L}$ annexin V binding solution and analyzed by flow cytometry.

RNA Extraction and Real-time PCR. Total RNA was extracted with the EZ-press RNA Purification Kit (B0004DP) on the basis of manufacturer's prospectus. Reverse transcription was performed in a $20 \mu \mathrm{L}$ reaction volume with the total $2 \mu \mathrm{g}$ of RNA using HiScript II Q RT SuperMix (\#R222-01, Vazyme, Nanjing, China). Real -time PCR were performed with SYBR Green Master Mix (\#Q121-02, Vazyme) according to the manufacture's recommended protocol.

The following sequences of primers were designed to amplify PCR product:

GAPDH:

5'-GAGTCCACTGGCGTCTTCAC-3'

5'-TTCACACCCATGACGAACAT-3'

PAK1:

5'-GGTTTCAAGTGTTTAGTAACTTTTCCA-3'

5'-TTAGCTGCAGCAATCAGTGG-3'

RRAS2:

5'-GAGCAGCCCGGCTAGATATT-3'

5'-TGTTCTCTCATGGCTCCAAA-3'

TGFA:

5'-CCTGGCTGTCCTTATCATCAC-3'

5'-GGCACCACTCACAGTGTTTTC-3'

TEMEL2:

5'-CCACAGGGTTGCGTCTCAG-3' 
5'-GGAGCACTGCACAGACAGAG-3'

HSPG2:

5'-CCAAATGCGCTGGACACATTC-3'

5'-CGGACACCTCTCGGAACTCT-3'

NCAM1:

5'-GGCATTTACAAGTGTGTGGTTAC-3'

5'-TTGGCGCATTCTTGAACATGA-3'

\section{ASSOCIATED CONTENT}

\section{Supporting information}

Molecular formula strings (CSV).

Diffraction data and structure refinement statistics of crystallography; extended data series for tested compounds (PDF)

\section{Accession Codes}

The PDB code for NSD2-PWWP1 bound with compound 5 is 7VLN. The authors will release the atomic coordinates and experimental data upon article publication.

\section{AUTHOR INFORMATION}

\section{Corresponding Authors}

Tongchao Liu-Department of Medicinal Chemistry, Shanghai Institute of Materia

Medica, Chinese Academy of Sciences, 555 Zuchongzhi Road, Shanghai 201203, P. R.

China; Email: tongchao_liu@simm.ac.cn

Xun Huang - Division of Anti-tumor Pharmacology, State Key Laboratory of Drug

Research, Shanghai Institute of Materia Medica, Chinese Academy of Sciences, Shanghai 201203, P. R. China; University of Chinese Academy of Sciences, Beijing 
100049, P. R. China; School of Pharmaceutical Science and Technology, Hangzhou Institute for Advanced Study, UCAS, Hangzhou 310024, P. R. China; Phone:+86 21 50806600; Email: xhuang@simm.ac.cn

Bing Xiong - Department of Medicinal Chemistry, Shanghai Institute of Materia Medica, Chinese Academy of Sciences, Shanghai 201203, P. R. China; University of Chinese Academy of Sciences, Beijing 100049, P. R. China; orcid.org/ 0000-0001-9776-8136; Phone: +86 21 50807088; Email: bxiong@simm.ac.cn

Shilin Xu - Drug Discovery \& Development Center, State Key Laboratory of Drug Research, Shanghai Institute of Materia Medica, Chinese Academy of Sciences, \#555 Zu Chong Zhi Road, Shanghai, 201203, China; University of Chinese Academy of Sciences, Beijing 100049, P. R. China; Email: slxu@simm.ac.cn

\section{Authors}

Na Li - Department of Medicinal Chemistry, Shanghai Institute of Materia Medica,

Chinese Academy of Sciences, 555 Zuchongzhi Road, Shanghai 201203, P. R. China; University of Chinese Academy of Sciences, NO.19A Yuquan Road, Beijing 100049, P. R. China

Hong Yang - Division of Antitumor Pharmacology, State Key Laboratory of Drug Research, Shanghai Institute of Materia Medica, Chinese Academy of Sciences, 555 Zuchongzhi Road, Shanghai 201203, P. R. China

Ke Liu - Shanghai Synchrotron Radiation Facility, Shanghai Advanced Research Institute, Chinese Academy of Sciences, 239 Zhangheng Road, Shanghai 201210, China

Liwei Zhou - Department of Medicinal Chemistry, Shanghai Institute of Materia 
Medica, Chinese Academy of Sciences, 555 Zuchongzhi Road, Shanghai 201203, P. R. China

Yuting Huang - Division of Antitumor Pharmacology, State Key Laboratory of Drug Research, Shanghai Institute of Materia Medica, Chinese Academy of Sciences, 555 Zuchongzhi Road, Shanghai 201203, P. R. China

Danyan Cao - Department of Medicinal Chemistry, Shanghai Institute of Materia Medica, Chinese Academy of Sciences, 555 Zuchongzhi Road, Shanghai 201203, P. R. China

Yanlian Li - Department of Medicinal Chemistry, Shanghai Institute of Materia Medica, Chinese Academy of Sciences, 555 Zuchongzhi Road, Shanghai 201203, P. R. China

Yaoliang Sun - Drug Discovery \& Development Center, State Key Laboratory of Drug Research, Shanghai Institute of Materia Medica, Chinese Academy of Sciences, \#555 Zu Chong Zhi Road, Shanghai, 201203, China

Aisong Yu - Division of Antitumor Pharmacology, State Key Laboratory of Drug Research, Shanghai Institute of Materia Medica, Chinese Academy of Sciences, 555 Zuchongzhi Road, Shanghai 201203, P. R. China

Zhiyan Du - Department of Medicinal Chemistry, Shanghai Institute of Materia Medica, Chinese Academy of Sciences, 555 Zuchongzhi Road, Shanghai 201203, P. R. China

Huan Zhou - Shanghai Synchrotron Radiation Facility, Shanghai Advanced Research Institute, Chinese Academy of Sciences, 239 Zhangheng Road, Shanghai 
201210, China

Ying Zhang - Division of Antitumor Pharmacology, State Key Laboratory of Drug

Research, Shanghai Institute of Materia Medica, Chinese Academy of Sciences, 555 Zuchongzhi Road, Shanghai 201203, P. R. China

Bingyang Wang - Drug Discovery \& Development Center, State Key Laboratory of Drug Research, Shanghai Institute of Materia Medica, Chinese Academy of Sciences, \#555 Zu Chong Zhi Road, Shanghai, 201203, China

Meiyu Geng - Division of Anti-tumor Pharmacology, State Key Laboratory of Drug Research, Shanghai Institute of Materia Medica, Chinese Academy of Sciences, Shanghai 201203, P. R. China; University of Chinese Academy of Sciences, Beijing 100049, P. R. China; School of Pharmaceutical Science and Technology, Hangzhou Institute for Advanced Study, UCAS, Hangzhou 310024, P. R. China

\section{Author Contributions}

N. L. designed and synthesized the compounds. H. Y. conducted the biological experiments and analyzed the results. K. L. resolved the co-crystal structure of NSD2-PWWP1 and compound 5. L. Z. helped to design the molecules and synthetic route. These authors contributed significantly to this project, so we deemed them as equal contribution.

Y. S. and B. W. helped to design the molecules and synthetic route. D. C. and Y. L. conducted the enzymatic assay. Y. H., A. Y. and Y. Z. participated in the biological assays. D. C., Z. D. and H. Z. conducted the co-crystal structure of compounds with X-ray crystallography. M. G. helped to design the project. 
N. L., H. Y. and K. L. prepared the initial manuscript. T. L., X. H., S. X. and B. X. designed the project and wrote the manuscript. All of the authors read and approved the final manuscript.

\section{Notes}

The authors declare no competing financial interest.

\section{ACKNOWLEDGMENTS}

We are grateful for financial support from the National Natural Science Foundation of China (Grant 21922707 and 81773572), the Major projects of National Natural Science Foundation of China (Grant 81991523), the Collaborative Innovation Cluster Project of Shanghai Municipal Commission of Health and Family Planning (Grant 2019CXJQ02), the Natural Science Foundation of Shanghai (21ZR1475500). Open Program of State Key Laboratory of new Drug development (Grant SIMM2105KF-04), SA-SIBS Scholarship Program and Shanghai Municipal Science and Technology Major Project. This work was carried out with the support of Shanghai Synchrotron Radiation Facility. We also thank Prof. Feng Yu and Prof. Jian Li for the assistance in determination of co-crystal structure.

\section{REFERENCES}

1. Vougiouklakis, T.; Hamamoto, R.; Nakamura, Y.; Saloura, V. The NSD family of protein methyltransferases in human cancer. Epigenomics 2015, 7, 863-74.

2. Bennett, R. L.; Swaroop, A.; Troche, C.; Licht, J. D. The Role of Nuclear Receptor-Binding SET Domain Family Histone Lysine Methyltransferases in Cancer. Cold Spring Harb Perspect Med 2017, 7 .

3. Cheong, C. M.; Mrozik, K. M.; Hewett, D. R.; Bell, E.; Panagopoulos, V.; Noll, J. E.; Licht, J. 
D.; Gronthos, S.; Zannettino, A. C. W.; Vandyke, K. Twist-1 is upregulated by NSD2 and contributes to tumour dissemination and an epithelial-mesenchymal transition-like gene expression signature in t(4;14)-positive multiple myeloma. Cancer Lett 2020, 475, 99-108.

4. Pierro, J.; Saliba, J.; Narang, S.; Sethia, G.; Saint Fleur-Lominy, S.; Chowdhury, A.; Qualls, A.; Fay, H.; Kilberg, H. L.; Moriyama, T.; Fuller, T. J.; Teachey, D. T.; Schmiegelow, K.; Yang, J. J.; Loh, M. L.; Brown, P. A.; Zhang, J.; Ma, X.; Tsirigos, A.; Evensen, N. A.; Carroll, W. L. The NSD2 p.E1099K Mutation Is Enriched at Relapse and Confers Drug Resistance in a Cell Context-Dependent Manner in Pediatric Acute Lymphoblastic Leukemia. Mol Cancer Res 2020, $18,1153-1165$.

5. Chen, R.; Chen, Y.; Zhao, W.; Fang, C.; Zhou, W.; Yang, X.; Ji, M. The Role of Methyltransferase NSD2 as a Potential Oncogene in Human Solid Tumors. Onco Targets Ther 2020, 13, 6837-6846.

6. Sengupta, D.; Zeng, L.; Li, Y.; Hausmann, S.; Ghosh, D.; Yuan, G.; Nguyen, T. N.; Lyu, R.; Caporicci, M.; Morales Benitez, A.; Coles, G. L.; Kharchenko, V.; Czaban, I.; Azhibek, D.; Fischle, W.; Jaremko, M.; Wistuba, II; Sage, J.; Jaremko, L.; Li, W.; Mazur, P. K.; Gozani, O. NSD2 dimethylation at H3K36 promotes lung adenocarcinoma pathogenesis. Mol Cell 2021.

7. Weinberg, D. N.; Papillon-Cavanagh, S.; Chen, H.; Yue, Y.; Chen, X.; Rajagopalan, K. N.; Horth, C.; McGuire, J. T.; Xu, X.; Nikbakht, H.; Lemiesz, A. E.; Marchione, D. M.; Marunde, M. R.; Meiners, M. J.; Cheek, M. A.; Keogh, M. C.; Bareke, E.; Djedid, A.; Harutyunyan, A. S.; Jabado, N.; Garcia, B. A.; Li, H.; Allis, C. D.; Majewski, J.; Lu, C. The histone mark H3K36me2 recruits DNMT3A and shapes the intergenic DNA methylation landscape. Nature 2019, 573, 281-286. 
8. Yuan, S.; Natesan, R.; Sanchez-Rivera, F. J.; Li, J.; Bhanu, N. V.; Yamazoe, T.; Lin, J. H.; Merrell, A. J.; Sela, Y.; Thomas, S. K.; Jiang, Y.; Plesset, J. B.; Miller, E. M.; Shi, J.; Garcia, B. A.; Lowe, S. W.; Asangani, I. A.; Stanger, B. Z. Global Regulation of the Histone Mark H3K36me2 Underlies Epithelial Plasticity and Metastatic Progression. Cancer Discov 2020, 10, 854-871.

9. Huang, H.; Howard, C. A.; Zari, S.; Cho, H. J.; Shukla, S.; Li, H.; Ndoj, J.; Gonzalez-Alonso, P.; Nikolaidis, C.; Abbott, J.; Rogawski, D. S.; Potopnyk, M. A.; Kempinska, K.; Miao, H.; Purohit, T.; Henderson, A.; Mapp, A.; Sulis, M. L.; Ferrando, A.; Grembecka, J.; Cierpicki, T. Covalent inhibition of NSD1 histone methyltransferase. Nat Chem Biol 2020, 16, 1403-1410.

10. Bottcher, J.; Dilworth, D.; Reiser, U.; Neumuller, R. A.; Schleicher, M.; Petronczki, M.; Zeeb, M.; Mischerikow, N.; Allali-Hassani, A.; Szewczyk, M. M.; Li, F.; Kennedy, S.; Vedadi, M.; Barsyte-Lovejoy, D.; Brown, P. J.; Huber, K. V. M.; Rogers, C. M.; Wells, C. I.; Fedorov, O.; Rumpel, K.; Zoephel, A.; Mayer, M.; Wunberg, T.; Bose, D.; Zahn, S.; Arnhof, H.; Berger, H.; Reiser, C.; Hormann, A.; Krammer, T.; Corcokovic, M.; Sharps, B.; Winkler, S.; Haring, D.; Cockcroft, X. L.; Fuchs, J. E.; Mullauer, B.; Weiss-Puxbaum, A.; Gerstberger, T.; Boehmelt, G.; Vakoc, C. R.; Arrowsmith, C. H.; Pearson, M.; McConnell, D. B. Fragment-based discovery of a chemical probe for the PWWP1 domain of NSD3. Nat Chem Biol 2019, 15, 822-829.

11. Coussens, N. P.; Kales, S. C.; Henderson, M. J.; Lee, O. W.; Horiuchi, K. Y.; Wang, Y.; Chen, Q.; Kuznetsova, E.; Wu, J.; Chakka, S.; Cheff, D. M.; Cheng, K. C.; Shinn, P.; Brimacombe, K. R.; Shen, M.; Simeonov, A.; Lal-Nag, M.; Ma, H.; Jadhav, A.; Hall, M. D. High-throughput screening with nucleosome substrate identifies small-molecule inhibitors of the human histone lysine methyltransferase NSD2. J Biol Chem 2018, 293, 13750-13765.

12. Ferreira de Freitas, R.; Liu, Y.; Szewczyk, M. M.; Mehta, N.; Li, F.; McLeod, D.; 
Zepeda-Velazquez, C.; Dilworth, D.; Hanley, R. P.; Gibson, E.; Brown, P. J.; Al-Awar, R.; James, L. I.; Arrowsmith, C. H.; Barsyte-Lovejoy, D.; Min, J.; Vedadi, M.; Schapira, M.; Allali-Hassani, A. Discovery of Small-Molecule Antagonists of the PWWP Domain of NSD2.J Med Chem 2021, $64,1584-1592$.

13. Dilworth, D.; Hanley, R. P.; Ferreira de Freitas, R.; Allali-Hassani, A.; Zhou, M.; Mehta, N.; Marunde, M. R.; Ackloo, S.; Carvalho Machado, R. A.; Khalili Yazdi, A.; Owens, D. D. G.; Vu, V.; Nie, D. Y.; Alqazzaz, M.; Marcon, E.; Li, F.; Chau, I.; Bolotokova, A.; Qin, S.; Lei, M.; Liu, Y.; Szewczyk, M. M.; Dong, A.; Kazemzadeh, S.; Abramyan, T.; Popova, I. K.; Hall, N. W.; Meiners, M. J.; Cheek, M. A.; Gibson, E.; Kireev, D.; Greenblatt, J. F.; Keogh, M. C.; Min, J.; Brown, P. J.; Vedadi, M.; Arrowsmith, C. H.; Barsyte-Lovejoy, D.; James, L. I.; Schapira, M. A chemical probe targeting the PWWP domain alters NSD2 nucleolar localization. Nat Chem Biol 2021.

14. Sankaran, S. M.; Wilkinson, A. W.; Elias, J. E.; Gozani, O. A PWWP Domain of Histone-Lysine N-Methyltransferase NSD2 Binds to Dimethylated Lys-36 of Histone H3 and Regulates NSD2 Function at Chromatin. J Biol Chem 2016, 291, 8465-74.

15. Wang, S.; Yang, H.; Su, M.; Lian, F.; Cong, Z.; Wei, R.; Zhou, Y.; Li, X.; Zheng, X.; Li, C.; Fu, X.; Han, X.; Shi, Q.; Li, C.; Zhang, N.; Geng, M.; Liu, H.; Li, J.; Huang, X.; Wang, J. 5-Aminonaphthalene derivatives as selective nonnucleoside nuclear receptor binding SET domain-protein 2 (NSD2) inhibitors for the treatment of multiple myeloma. Eur J Med Chem 2021, 222,113592 .

16. Wang, Q.; Yu, F.; Cui, Y.; Zhang, K.; Pan, Q.; Zhong, C.; Liu, K.; Zhou, H.; Sun, B.; He, J. Mini-beam modes on standard MX beamline BL17U at SSRF. Rev Sci Instrum 2017, 88, 073301. 
17. Kabsch, W. Processing of X-ray snapshots from crystals in random orientations. Acta Crystallogr D Biol Crystallogr 2014, 70, 2204-16.

18. Adams, P. D.; Afonine, P. V.; Bunkoczi, G.; Chen, V. B.; Echols, N.; Headd, J. J.; Hung, L. W.; Jain, S.; Kapral, G. J.; Grosse Kunstleve, R. W.; McCoy, A. J.; Moriarty, N. W.; Oeffner, R. D.; Read, R. J.; Richardson, D. C.; Richardson, J. S.; Terwilliger, T. C.; Zwart, P. H. The Phenix software for automated determination of macromolecular structures. Methods 2011, 55, 94-106.

19. Kovalevskiy, O.; Nicholls, R. A.; Long, F.; Carlon, A.; Murshudov, G. N. Overview of refinement procedures within REFMAC5: utilizing data from different sources. Acta Crystallogr D Struct Biol 2018, 74, 215-227.

20. Emsley, P.; Lohkamp, B.; Scott, W. G.; Cowtan, K. Features and development of Coot. Acta Crystallogr D Biol Crystallogr 2010, 66, 486-501. 


\section{Table of Contents Graphic}

Structure-based Discovery of NSD2-PWWP1 Inhibitors

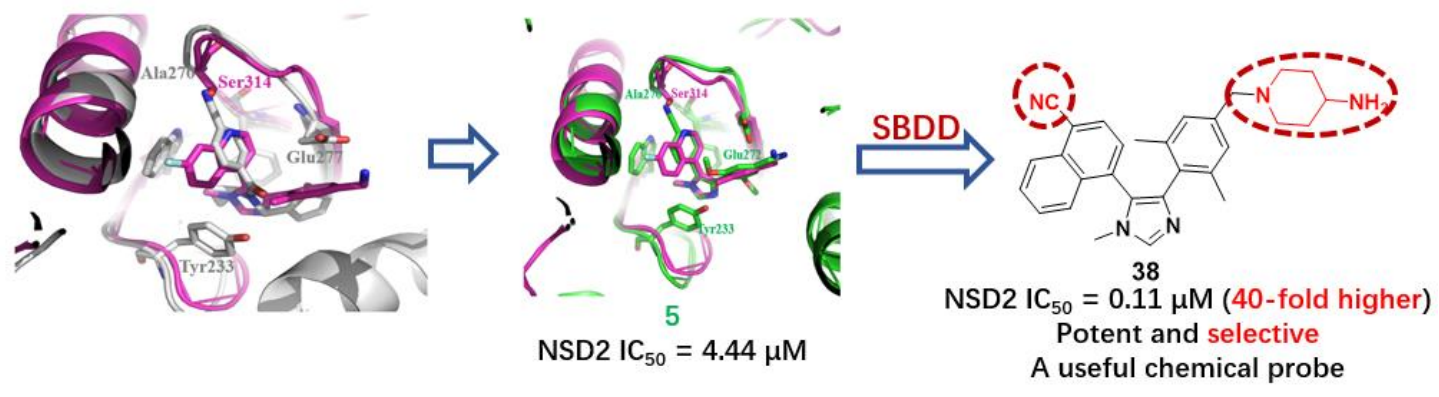

